\title{
Gravity waves from soft theorem in general dimensions
}

\author{
Alok Laddha ${ }^{a}$ and Ashoke Sen ${ }^{b}$ \\ ${ }^{a}$ Chennai Mathematical Institute, \\ Siruseri, Chennai, India \\ ${ }^{b}$ Harish-Chandra Research Institute, HBNI, \\ Chhatnag Road, Jhusi, Allahabad 211019, India \\ E-mail: aladdha@cmi.ac.in, sen@mri.ernet.in
}

ABStract: Classical limit of multiple soft graviton theorem can be used to compute the angular power spectrum of long wavelength gravitational radiation in classical scattering provided the total energy carried away by the radiation is small compared to the energies of the scatterers. We could ensure this either by taking the limit in which the impact parameter is large compared to the Schwarzschild radii of the scatterers, or by taking the probe limit where one object (the probe) has mass much smaller than the other object (the scatterer). We compute the results to subsubleading order in soft momentum and test them using explicit examples involving classical scattering. Our analysis also generalizes to the case where there are multiple objects involved in the scattering and the objects exchange mass, fragment or fuse into each other during the scattering. A similar analysis can be carried out for soft photons to subleading order, reproducing standard textbook results. We also discuss the modification of soft expansion in four dimensions beyond the leading order due to infrared divergences.

KeYwords: Classical Theories of Gravity, Scattering Amplitudes

ARXiv EPrint: 1801.07719 


\section{Contents}

1 Introduction and summary 1

2 Electromagnetic radiation from classical scattering 5

$\begin{array}{llll}3 & \text { Gravitational radiation from classical scattering } & 7\end{array}$

4 Sum over polarizations for probe scattering 16

$\begin{array}{lll}5 & \text { Plunge } & 17\end{array}$

6 Universal parts of higher order non-universal results 18

$\begin{array}{lll}6.1 & \text { Subleading soft photon theorem } & 18\end{array}$

$\begin{array}{ll}\text { 6.2 Subsubleading soft graviton theorem } & 19\end{array}$

$\begin{array}{ll}6.3 \text { Hidden assumptions } & 20\end{array}$

$\begin{array}{lll}7 & \text { Tests of soft theorem from classical radiation analysis } & \mathbf{2 0}\end{array}$

$\begin{array}{lll}7.1 & \text { Soft photon theorem } & 21\end{array}$

$\begin{array}{ll}7.2 & \text { Soft graviton theorem } \\ 7.2 .1\end{array}$

7.2.1 Scattering via fusion and decay: 24

$\begin{array}{lll}\text { 7.2.2 } & \text { Scattering via multiple fusion and decay } & 28\end{array}$

$\begin{array}{lll}7.2 .3 & \text { Inclusion of spin } & 32\end{array}$

$\begin{array}{lll}\text { 7.2.4 Probe scattering from a charged scatterer } & 34\end{array}$

$\begin{array}{lll}\text { 7.2.5 Probe scattering from Schwarzschild black hole } & 39\end{array}$

$\begin{array}{lll}7.2 .6 & \text { Scattering on the brane-world } & 41\end{array}$

8 Modification of soft expansion in four dimensions 42

9 Discussion $\quad 44$

A Alternative analysis of the test of soft photon theorem in four dimensions 46

\section{Introduction and summary}

Soft theorems have a long history [1-12]. They express amplitudes with one or more low momentum photon or graviton in terms of amplitudes without such photons or gravitons. Recent surge of interest in soft theorem began with the realization that they are related to asymptotic symmetries [13-30]. During this investigation it has also become clear that soft theorems extend beyond leading order in the soft momentum [31-75].

There now exists general diagrammatic proof of soft graviton theorem that is valid for generic finite energy external states in generic theories in generic number of dimensions [7678]. In particular these results also hold when the external states represent composite objects. Up to subleading order in the soft momenta the result is universal, i.e. the relation between the amplitude with soft gravitons and the amplitude without soft gravitons is 
independent of the theory or the type of particles we have in the external state, and is sensitive only to the momenta and angular momenta carried by these particles. At the subsubleading order there still exists a relation between the two amplitudes, but it is not universal, i.e. it depends on the theory and also the nature of the external particles involved $[57,72,77]$. The analysis of $[76-78]$ can be easily extended to the electromagnetic case following [74], but in this case the soft theorem exists only to subleading order, and only the leading term is universal.

Since soft theorems hold for all external states, they also hold for classical objects carrying large mass or charge. Therefore by taking appropriate limit of the soft theorem we should be able to generate universal formula for electromagnetic and / or gravitational radiation during a classical scattering process that depends only on the initial and final momenta and angular momenta of the objects being scattered, but not on the details of the scattering. For leading soft theorem this has already been studied and shown to agree with classical results [8]. Our goal will be to use the subleading and subsubleading soft graviton theorem on the scattering of a pair of massive objects, with classical limit corresponding to their masses being much larger than the Planck mass. We shall also see that a similar analysis for soft photons reproduce the standard textbook results for radiation from a moving charge [79] to subleading order.

Our results will be valid for graviton wave-lengths large compared to the impact parameter and the internal sizes of the scatterers. The validity of our analysis also requires that the total radiated energy is small compared to the energy of the scatterers themselves. We can achieve this by taking the limit in which the impact parameter or the typical size of the scattarers is large compared to the Schwarzschild radii of the scatterers. On the other hand if we take the impact parameter and the sizes to be of the order of the Schwarzschild radii, then we need to work in the approximation in which one of the objects (called the probe) has mass small compared to the other (called the scatterer).

For the probe-scatterer approximation, our results in $D$ space-time dimensions can be summarized as follows. We shall work in the frame in which the scatterer is at rest initially. Up to subleading order in $\omega$, the total energy carried by gravity waves of polarization $\varepsilon$, with frequency lying between $\omega$ and $\omega(1+\delta)$ and within a solid angle $\Delta \Omega$ around the unit vector $\hat{n}$ pointing out of the scatterer is given by

$$
\frac{1}{2^{D} \pi^{D-1}}\left|S_{\mathrm{gr}}^{(0)}(\varepsilon, k)+S_{\mathrm{gr}}^{(1)}(\varepsilon, k)\right|^{2} \omega^{D-1} \Delta \Omega \delta
$$

where we have used $\hbar=c=8 \pi G_{N}=1$ units, $k=-(\omega, \omega \hat{n})$ denotes the momentum carried by the individual gravitons, and ${ }^{1}$

$$
\begin{aligned}
S_{\mathrm{gr}}^{(0)} & =\sum_{a=1}^{2}\left[\frac{\varepsilon_{\mu \nu} p_{(a)}^{\mu} p_{(a)}^{\nu}}{p_{(a)} \cdot k}+\varepsilon_{00} \frac{p_{(a)} \cdot k}{\left(k^{0}\right)^{2}}+2 \varepsilon_{0 \nu} \frac{p_{(a)}^{\nu}}{k^{0}}\right], \\
S_{\mathrm{gr}}^{(1)} & =i \sum_{a=1}^{2}\left[\left\{\frac{\varepsilon_{\mu \nu} p_{(a)}^{\mu} k_{\rho}}{p_{(a)} \cdot k}+\frac{\varepsilon_{\nu 0} k_{\rho}}{k^{0}}\right\} \mathbf{j}_{(a)}^{\rho \nu}+\frac{\mathbf{J}^{j i}}{M_{0}}\left\{\frac{\varepsilon_{i 0} k_{j} p_{(a)} \cdot k}{\left(k^{0}\right)^{2}}+\frac{\varepsilon_{i \nu} p_{(a)}^{\nu} k_{j}}{k^{0}}\right\}\right] .
\end{aligned}
$$

\footnotetext{
${ }^{1}$ The factor given in (1.2) has previously appeared in [80] in a different context.
} 
$p_{(1)}$ and $p_{(2)}$ are the momenta of the probe before and after the scattering, with the sign convention that momentum is always measured in the ingoing direction. The indices are raised and lowered by flat metric $\eta^{\mu \nu}$ and $\eta_{\mu \nu}$ with mostly plus signature. The scatterer is initially taken to be at rest. $\mathbf{J}$ is the initial angular momentum of the scatterer and $M_{0}$ is the mass of the scatterer. The indices $i, j, \ell$ run over spatial coordinates and the indices $\mu, \nu, \rho$ run over all space-time coordinates. $\mathbf{j}_{(1)}$ and $\mathbf{j}_{(2)}$ are the angular momenta of the probe before and after the scattering, measured with respect to the point where the scatterer is situated initially, ${ }^{2}$ again with the convention that they are counted with positive sign for ingoing and negative sign for outgoing particles. Under the assumption that the internal size of the probe is small compared to the impact parameter, $\mathbf{j}_{(a)}$ 's receive dominant contribution only from the orbital angular momenta. The formula for $\mathbf{j}_{(1)}$ and $\mathbf{j}_{(2)}$ in terms of the initial and final particle trajectories can be found in (3.32), (3.33). (1.1) can be shown to be invariant under the gauge transformation that shifts $\varepsilon_{\mu \nu}$ by $k_{\mu} \xi_{\nu}+\xi_{\mu} k_{\nu}$ for some vector $\xi$. It is also invariant under a shift in the origin of the time coordinate under which $\mathbf{j}_{(a)}^{0 i}=-\mathbf{j}_{(a)}^{i 0}$ gets shifted by $c^{0} p_{(a)}^{i}$ for some constant $c^{0}$. The expansion is in powers of $\max (\omega a, \omega b)$, where $b$ is the impact parameter and $a$ is the maximum of the sizes of the probe and the scatterer. $S_{\mathrm{gr}}^{(0)}$ represents the leading term in the expansion and $S_{\mathrm{gr}}^{(1)}$ is the subleading term. Since for real $\varepsilon S_{\mathrm{gr}}^{(1)}$ is purely imaginary, its effect on (1.1) vanishes to subleading order. However for circular polarization for which $\varepsilon$ is complex, $S_{\mathrm{gr}}^{(1)}$ contributes to subleading order.

Our analysis is also valid when the probe fuses to the scatterer. In this case we have to set $p_{(2)}$ and $\mathbf{j}_{(2)}$ to 0 in (1.2), (1.3). It is also valid when there is more that one probe and / or when the probe splits up into fragments producing more than one object in the final state and / or exchanges mass with the scatterer i.e. we do not need to demand that the initial and final masses of the objects coincide. In all these case we have to extend the sum over $a$ in (1.2), (1.3) to all initial and final state objects of finite energy.

If we do not use the probe-scatterer approximation, but consider a general scattering with arbitrary number of incoming and outgoing objects carrying energies of the same order, then we can still use (1.1)-(1.3) for large impact parameter or large size, but we need to allow the sum over $a$ to run over all external particles. To the experts this may appear somewhat unfamiliar since the usual soft theorem does not have the non-covariant terms in (1.2), (1.3) involving $\varepsilon_{00}$ and $\varepsilon_{0 \nu}$. It is easy to see however that when all external states are included in the sum over $a$, the contribution from these terms vanish using conservation of total momentum and total angular momentum. The same argument shows that the term involving the $\mathbf{J}^{i j} / M_{0}$ vanishes. This is just as well since now there is no distinguished scatterer whose angular momentum represents $\mathbf{J}^{i j}$.

(1.2), (1.3) are universal independent of the type of probe or scatterer we have. For example the scatterer could be Kerr-Newmann black hole in the Einstein-Maxwell theory, or more general rotating black hole in a theory of gravity coupled of other massless gauge fields and scalars, or some other compact massive (rotating) object. The probe could be any object with mass large compared to the Planck mass but much smaller than that of

\footnotetext{
${ }^{2}$ More precisely, this is the center of momentum of the scatterer around which $\mathbf{J}^{i 0}$ components vanish.
} 
the scatterer. We expect the universality to break down at the next order in the expansion in powers of $\omega a$ where the results will be sensitive to the internal structure of the scatterer. However we can still make some meaningful prediction if we take the impact parameter $b$ to be much larger than the size $a$. In this case the non-universal terms give corrections of order $\omega^{2} a^{2}$, whereas the universal terms have larger contribution of order $\omega^{2} b^{2}$ and $\omega^{2} a b$. These universal contributions are captured by adding to $S_{\mathrm{gr}}^{(0)}+S_{\mathrm{gr}}^{(1)}$ in (1.1) a third term

$$
S_{\mathrm{gr}}^{(2)}=-\frac{1}{2} \sum_{a=1}^{2}\left(p_{(a)} \cdot k\right)^{-1} \varepsilon_{\mu \rho} k_{\nu} k_{\sigma} \mathbf{j}_{(a)}^{\mu \nu} \mathbf{j}_{(a)}^{\rho \sigma}-\sum_{a=1}^{2} \frac{1}{M_{0} k^{0}} \varepsilon_{i \rho} k_{j} k_{\sigma} \mathbf{J}^{i j} \mathbf{j}_{(a)}^{\rho \sigma} .
$$

If the macroscopic objects have energies that are comparable to each other, then eq. (1.4) still holds, but the sum over $a$ runs over all the external states. In this case the contribution from the second term in (1.4) vanishes by conservation of angular momentum. Also if we express the $\mathbf{j}_{(a)}^{\mu \nu}$ 's as sum of spin and orbital angular momenta, then the terms quadratic in the orbital angular momenta and the cross terms between the orbital and the spin angular momenta are of order $\omega^{2} b^{2}$ and $\omega^{2} a b$ and are large compared to the non-universal terms. However the term proportional to the square of the spin angular mometum is of order $\omega^{2} a^{2}$ and are of the same order as the non-universal terms. Therefore we need to ignore this term in (1.4).

Finally, by combining the result on the angular power spectrum described above with the result of explicit computation given in section 7.2, we can write down the expression for the radiative part of the metric field $h_{\alpha \beta} \equiv\left(g_{\alpha \beta}-\eta_{\alpha \beta}\right) / 2$ for long wavelengths. Denoting by $\tilde{h}_{\alpha \beta}(\omega, \vec{x})$ the time Fourier transform of $h_{\alpha, \beta}(t, \vec{x})$, we have, for large $|\vec{x}|$ and up to subsubleading order in $\omega$,

$$
\begin{aligned}
\tilde{h}_{\alpha \beta}(\omega, \vec{x}) & =\tilde{e}_{\alpha \beta}(\omega, \vec{x})-\frac{1}{D-2} \eta_{\alpha \beta} \tilde{e}_{\gamma}^{\gamma}(\omega, \vec{x}), \\
\varepsilon^{\alpha \beta} \tilde{e}_{\alpha \beta}(\omega, \vec{x}) & =\mathcal{N} e^{i \omega R}\left[S_{\mathrm{gr}}^{(0)}(\varepsilon, k)+S_{\mathrm{gr}}^{(1)}(\varepsilon, k)+S_{\mathrm{gr}}^{(2)}(\varepsilon, k)\right], \\
R & \equiv|\vec{x}|, \quad \mathcal{N} \equiv\left(\frac{\omega}{2 \pi i R}\right)^{(D-2) / 2} \frac{1}{2 \omega},
\end{aligned}
$$

up to gauge transformation. ${ }^{3}$

A similar formula for electrodynamics exists up to subleading order in powers of the soft momentum, and is obtained by replacing $S_{\mathrm{gr}}^{(i)}$ by $S_{\mathrm{em}}^{(i)}$ given in (2.2) and (6.2). Both for electromagnetism and gravity the relation between classical radiation and soft theorem at the leading order has been noted before, see e.g. [79] for electromagnetism and [85] for gravity. However this relation at the subleading and subsubleading order does not seem to have been explored in detail before. ${ }^{4}$

\footnotetext{
${ }^{3}$ The additional power of $\omega$ coming from $\mathcal{N}$ for $D>4$ is responsible for the absence of conventional memory effect in $D>4$ [81-83]. In [84] a new definition of memory was proposed based on the "Coloumbic modes" of $\tilde{h}_{\alpha \beta}$ that fall off as $1 / R^{D-3}$. It would be interesting to extend our analysis to this order in $1 / R$ and check that these Coloumbic modes indeed have a non-trivial zero frequency limit.

${ }^{4}$ The relation between subleading terms in the soft graviton theorem and a new kind of memory effect in four space-time dimensions was discussed in [86]. However, as we shall discuss in section 8, in four dimensions the usual soft expansion seems to get modified beyond leading order due to logarithmic singularities in the expansion.
} 
It may be possible to give a general proof of soft theorem in classical scattering using the effective field theory approach [87-89] (see [90] for a review). However in this paper we shall restrict our analysis to verifying the soft theorem explicitly in various classical scattering processes.

In four space-time dimensions soft theorems are expected to be modified due to infrared divergence effects [41]. We shall avoid these divergences by working in general dimensions. In section 8 we shall discuss the special case of four dimensions and explore how the classical limit of soft theorem is affected by the classical counterpart of infrared divergences - long range force acting on objects taking part in the scattering.

It was argued in $[16,84,86,91,92]$ that soft graviton theorem is related to memory effect [93-96]. This correspondence was established in two steps — first relating soft graviton theorem to the radiative field produced in the scattering and then relating the radiative field to the memory effect. The latter connection has so far been understood only in even space-time dimensions. In contrast the connection between soft theorem and power spectrum of gravitational radiation described in (1.1) makes use of the relation between radiative field and the power spectrum and holds in all space-time dimensions. Our analysis further shows that the first step in this relation - the relation between soft theorem and the classical radiative field - is based not on single soft graviton theorem but multiple soft graviton theorem, and relies on the vanishing of the contact term in the multiple soft theorem in the classical limit.

The rest of the paper is organized as follows. In section 2 we review the classical limit of the leading soft theorem in electrodynamics. In section 3 we apply this to soft theorem in gravity to subleading order. In section 4 we compute the angular power spectrum of soft gravitons by summing over polarizations and show that in the probe-scatterer approximation the effect of the scatterer does not fully decouple even in the infinite mass limit. In section 5 we consider a slightly different situation where the probe fuses with the scatterer to form a single object, and analyze the angular power spectrum of soft gravitons emitted during this process with the help of soft theorem. This will include in particular an object falling into the black hole. In section 6 we extend the analysis of classical limit of soft theorem to subleading order in electrodynamics and subsubleading order in gravity. Even though at this order the soft theorem receives non-universal corrections, we argue that in the limit of large impact parameter the universal part dominates over the non-universal part and we can make definite predictions. In section 7 we test the general formulae derived from soft theorem by analyzing the emission of electromagnetic and gravitational radiation during various classical scattering processes to respectively subleading and subsubleading order in soft momentum. In section 8 we explore the origin of the corrections to the soft theorem in four dimensions due to infrared effects. We conclude in section 9 with a discussion of our analysis.

\section{Electromagnetic radiation from classical scattering}

In this section we shall review the computation of long wavelength classical electromagnetic radiation for the scattering of charged particles using soft theorem. 
Let us consider the effect of emission of $M$ soft photons, carrying polarization and momenta $\left(\varepsilon_{1}, k_{1}\right), \cdots,\left(\varepsilon_{M}, k_{M}\right)$ during the scattering of $n$ finite energy particles carrying charge $q_{(a)}$ and momenta $p_{(a)}$ for $1 \leq a \leq n$. All momenta are regarded as ingoing so that an outgoing particle carries negative energy. According to the soft theorem, for small $\left\{k_{r}\right\}$, this amplitude is given by

$$
\left\{\prod_{r=1}^{M} S_{\mathrm{em}}^{(0)}\left(\varepsilon_{r}, k_{r}\right)\right\} \mathbf{M}
$$

where

$$
S_{\mathrm{em}}^{(0)}(\varepsilon, k)=\sum_{a=1}^{n} q_{(a)} \frac{\varepsilon \cdot p_{(a)}}{k \cdot p_{(a)}},
$$

and $\mathbf{M}$ is the amplitude of the process without soft gravitons. We shall now use this to derive a formula for the classical electromagnetic radiation from such a scattering process. Let us consider the differential cross section for emitting $N$ soft photons, each carrying polarization $\varepsilon$ and lying within a solid angle $\Delta \Omega$, and with frequency lying between $\omega$ and $\omega(1+\delta)$. This will be given by

$$
|\mathbf{M}|^{2} A^{N} / N !,
$$

where $A$ includes the contribution from the differential cross-section as well as the phase space factor:

$$
A \equiv \frac{1}{(2 \pi)^{D-1}} \frac{1}{2 \omega}\left|S_{\mathrm{em}}^{(0)}(\varepsilon, k)\right|^{2} \omega^{D-2} \omega \delta \Delta \Omega=\frac{1}{2^{D} \pi^{D-1}}\left|S_{\mathrm{em}}^{(0)}(\varepsilon, k)\right|^{2} \omega^{D-2} \Delta \Omega \delta .
$$

For $q_{(a)} \sim q$, the leading term in $S_{\mathrm{em}}^{(0)}(\varepsilon, k)$ goes as $q / \omega$. This gives $A \propto q^{2}$ for fixed $\omega, \Delta \Omega$ and $\delta$. Therefore $A$ is a large number for $q \gg 1$, which corresponds to the classical limit. (2.3) is maximized at

$$
\frac{\partial}{\partial N}(N \ln A-N \ln N+N)=0
$$

where, in anticipation of the fact that $N$ will turn out to be large at the maximum, we have used Stirling's formula for $N$ !. This gives

$$
N=A=\frac{1}{2^{D} \pi^{D-1}}\left|S_{\mathrm{em}}^{(0)}(\varepsilon, k)\right|^{2} \omega^{D-2} \Delta \Omega \delta .
$$

Before we proceed, let us make a few comments:

1. (2.3) is sharply peaked around the value of $N$ given in (2.6) with a width of order $\sqrt{N}$.

2. We could also allow emission of other soft photons in different momentum bins. This would multiply the $|\mathbf{M}|^{2}$ factor in (2.3) by other factors but will not affect the $N$ dependence of this expression. As a result the value of $N$ given in (2.6) remains unchanged.

Therefore (2.6) gives the number of soft photons emitted in this momentum bin.

We can also compute the total power carried by the soft photons in this bin by multiplying (2.6) by the energy of each soft photon. This is given by

$$
A \omega=\frac{1}{2^{D} \pi^{D-1}}\left|S_{\mathrm{em}}^{(0)}(\varepsilon, k)\right|^{2} \omega^{D-1} \Delta \Omega \delta .
$$


As a special case of the configuration discussed above, we can consider the probescatterer approximation in which we have a light particle (the probe) scattering off a heavy center (the scatterer). We begin with the scattering of two particles in $D$ space-time dimensions - one with mass $m$ and charge $q$ and the other with mass $M_{0}$ and charge $Q$. We label the momenta of the incoming and the outgoing particles as

$$
\begin{array}{ll}
p_{(1)}=\left(p_{(1)}^{0}, \vec{p}_{(1)}\right), & p_{(2)}=\left(p_{(2)}^{0}, \vec{p}_{(2)}\right), \\
p_{(3)}=\left(M_{0}, \overrightarrow{0}\right), & p_{(4)}=\left(-\sqrt{M_{0}^{2}+\vec{p}^{2}},-\vec{p}\right),
\end{array}
$$

where

$$
\vec{p} \equiv \vec{p}_{(1)}+\vec{p}_{(2)}, \quad p_{(1)}^{0}=\sqrt{\vec{p}_{(1)}^{2}+m^{2}}, \quad p_{(2)}^{0}=-\sqrt{\vec{p}_{(2)}^{2}+m^{2}} .
$$

Note that we have implemented the conservation of spatial momenta in the parametrization used in (2.8). We now take the limit $M_{0} \rightarrow \infty$ keeping $p_{(1)}, p_{(2)}$ finite. In this limit energy conservation equation reduces to

$$
p_{(1)}^{0}+p_{(2)}^{0}=0 .
$$

We denote by $\mathbf{M}$ the amplitude of this process. In this limit the $a=3$ and $a=4$ terms in the sum in (2.2) cancel and we are left with

$$
S_{\mathrm{em}}^{(0)}(\varepsilon, k)=q \sum_{a=1}^{2}(-1)^{a-1} \frac{\varepsilon \cdot p_{(a)}}{k \cdot p_{(a)}} .
$$

Substituting eq. (2.11) in (2.6) and taking $D=4$, we see that this agrees with eq. (15.3) of [79] after appropriate change in the units. ${ }^{5}$

Note that in the analysis given above we have taken $M_{0} \rightarrow \infty$ limit keeping $Q$ fixed. If instead we take the $M_{0} \rightarrow \infty$ limit keeping $Q / M_{0}$ fixed, as will be the case e.g. for scattering from a near extremal black hole, the soft factor $S_{\mathrm{em}}^{(0)}$ acquires an additional term

$$
\Delta S_{\mathrm{em}}^{(0)}=\frac{Q}{M_{0}} \frac{1}{k^{0}}\left(\varepsilon \cdot p-\varepsilon^{0} \frac{k \cdot p}{k^{0}}\right) .
$$

One can easily check the invariance of this term under the gauge transformation that shifts $\varepsilon^{\mu}$ by a vector proportional to $k^{\mu}$.

\section{Gravitational radiation from classical scattering}

We shall now repeat the analysis for emission of soft gravitons. Since there are several limits involved, we shall begin by describing the order of limits that we would like to take.

1. First we take limit in which the momenta / masses of all the incoming and outgoing objects become large, keeping their ratios fixed. We shall refer to them as macroscopic objects. This is the classical limit in which the number of emitted gravitons in any fixed frequency range becomes large.

\footnotetext{
${ }^{5}$ In our normalization of the charge the fine structure constant is given by $e^{2} /(4 \pi)$ where $e$ is the electric charge of the electron. In the normalization of [79] the fine structure constant is given by $e^{2}$. This leads to an extra factor of $4 \pi$ in the expression given in [79].
} 
2. The classical limit ensures that the number of soft gravitons emitted during the scattering is large. But we also need to ensure that the total energy carried by the radiation emitted during the scattering is small compared to the energies of individual macroscopic objects taking part in the scattering. As will be explained below (3.21), this is needed to ensure that in computing the soft factors we can only include the contribution due to the macroscopic objects and ignore the contribution due to the radiation that is produced during the scattering. One way to ensure this is to keep the distance between the centers of the macroscopic objects large compared to their Schwarzschild radii during the scattering. The other way is to take the probe limit. For $2 \rightarrow 2$ scattering this means that the mass of one of the incoming macroscopic objects (the scatterer) is taken to be large compared to the mass / energy of the other (the probe) in the rest frame of the scatterer. We shall see that in either of these cases, the ratio of the total energy radiated to the energies of the macroscopic objects becomes small.

3. Next we make the soft expansion - an expansion in powers of the momenta of soft gravitons. This will be an expansion in the larger of the two quantities $a \omega$ and $b \omega$ where $a$ denotes the typical size of the systems e.g. the Schwarzschild radius for a black hole, $b$ is the impact parameter and $\omega$ is the frequency of the soft graviton. Therefore we take $\omega a$ and $\omega b$ to be small. As we will see, at the leading and subleading order in the expansion, we can work with $b \sim a$. However for the subsubleading terms to be discussed in section 6 , the non-universal contributions are of order $(a \omega)^{2}$, and therefore to get a useful result without knowing the details of the internal structures of the probe and the scatterer, we shall take $b \gg a$. In this case terms of order $b^{2} \omega^{2}$ and $a b \omega^{2}$ are larger than the unknown terms of order $a^{2} \omega^{2}$ and we can make meaningful predictions.

In actual computation usage of soft theorem forces us to carry out the soft expansion first and then take the other limits. Due to this reverse order of limits we shall encounter some subtleties involving the difference between eqs. (3.8) and (3.9) below. We shall try to resolve this to the best of our ability.

In the soft limit the amplitude of a scattering process with soft gravitons is given by a soft factor acting on the amplitude without the soft graviton. For emission of $M$ soft gravitons of polarizations $\left(\varepsilon_{1}, k_{1}\right), \cdots,\left(\varepsilon_{M}, k_{M}\right)$, the leading term, replacing $(2.1),(2.2)$, is given by $[7,8]$

$$
\left\{\prod_{r=1}^{M} S_{\mathrm{gr}}^{(0)}\left(\varepsilon_{r}, k_{r}\right)\right\} \mathbf{M},
$$

where $\mathbf{M}$ is the amplitude without the soft gravitons, and

$$
S_{\mathrm{gr}}^{(0)}(\varepsilon, k) \equiv \sum_{a=1}^{n} \frac{\varepsilon_{\mu \nu} p_{(a)}^{\mu} p_{(a)}^{\nu}}{p_{(a)} \cdot k},
$$

where $p_{(a)}^{\mu}$ are the momenta of the macroscopic objects involved in the scattering. As before all momenta are counted as positive if ingoing. 
Let us now discuss subleading corrections. The amplitude with $M$ soft gravitons takes the following form to subleading order [78]

$$
\begin{aligned}
& {\left[\left\{\prod_{r=1}^{M} S_{\mathrm{gr}}^{(0)}\left(\varepsilon_{r}, k_{r}\right)\right\}+\sum_{s=1}^{M}\left\{\prod_{\substack{r=1 \\
r \neq s}}^{M} S_{\mathrm{gr}}^{(0)}\left(\varepsilon_{r}, k_{r}\right)\right\} S_{\mathrm{gr}}^{(1)}\left(\varepsilon_{s}, k_{s}\right)\right.} \\
& \left.+\sum_{\substack{r, u=1 \\
r<u}}^{M}\left\{\prod_{\substack{s=1 \\
s \neq r, u}}^{M} S_{\mathrm{gr}}^{(0)}\left(\varepsilon_{s}, k_{s}\right)\right\}\left\{\sum_{a=1}^{n}\left\{p_{(a)} \cdot\left(k_{r}+k_{u}\right)\right\}^{-1} \mathcal{M}\left(p_{(a)} ; \varepsilon_{r}, k_{r}, \varepsilon_{u}, k_{u}\right)\right\}\right] \mathbf{M} \\
& \mathcal{M}\left(p ; \varepsilon_{1}, k_{1}, \varepsilon_{2}, k_{2}\right)=\left(p . k_{1}\right)^{-1}\left(p . k_{2}\right)^{-1}\left\{-k_{1} . k_{2} p . \varepsilon_{1} \cdot p p . \varepsilon_{2} . p+2 p . k_{2} p . \varepsilon_{1} . p p . \varepsilon_{2} . k_{1}\right. \\
& \left.+2 p . k_{1} p . \varepsilon_{2} . p p \cdot \varepsilon_{1} . k_{2}-2 p . k_{1} p . k_{2} p . \varepsilon_{1} \cdot \varepsilon_{2} \cdot p\right\} \\
& +\left(k_{1} \cdot k_{2}\right)^{-1}\left\{-\left(k_{2} \cdot \varepsilon_{1} \cdot \varepsilon_{2} \cdot p\right)\left(k_{2} \cdot p\right)-\left(k_{1} \cdot \varepsilon_{2} \cdot \varepsilon_{1} \cdot p\right)\left(k_{1} \cdot p\right)\right. \\
& +\left(k_{2} \cdot \varepsilon_{1} \cdot \varepsilon_{2} \cdot p\right)\left(k_{1} \cdot p\right)+\left(k_{1} \cdot \varepsilon_{2} \cdot \varepsilon_{1} \cdot p\right)\left(k_{2} \cdot p\right)-\varepsilon_{1}^{\gamma \delta} \varepsilon_{2 \gamma \delta}\left(k_{1} \cdot p\right)\left(k_{2} \cdot p\right) \\
& \left.-2\left(p \cdot \varepsilon_{1} \cdot k_{2}\right)\left(p \cdot \varepsilon_{2} \cdot k_{1}\right)+\left(p \cdot \varepsilon_{2} \cdot p\right)\left(k_{2} \cdot \varepsilon_{1} \cdot k_{2}\right)+\left(p \cdot \varepsilon_{1} \cdot p\right)\left(k_{1} \cdot \varepsilon_{2} \cdot k_{1}\right)\right\}, \\
& S_{\mathrm{gr}}^{(1)}(\varepsilon, k)=\sum_{a=1}^{n} \frac{\varepsilon_{\mu \nu} p_{(a)}^{\mu} k_{\rho}}{p_{(a)} \cdot k} \mathcal{J}_{(a)}^{\rho \nu}, \quad \mathcal{J}^{\rho \nu} \equiv p^{\nu} \frac{\partial}{\partial p_{\rho}}-p^{\rho} \frac{\partial}{\partial p_{\nu}}+\Sigma^{\rho \nu},
\end{aligned}
$$

where $\Sigma^{\rho \nu}$ is the generator of rotation acting on the intrinsic spin of the state. When we take the convolution of this with the external wave-function $e^{-i p \cdot x}$, the $\partial / \partial p_{\rho}$, after integration by parts, produces a factor of $i x^{\rho}$. Therefore in the classical limit the first two terms in $\mathcal{J}^{\rho \nu}$ reduce to $i\left(x^{\rho} p^{\nu}-x^{\nu} p^{\rho}\right)$. Since $\left(x^{\rho} p^{\nu}-x^{\nu} p^{\rho}\right)$ is the $(\rho \nu)$ component of the orbital angular momentum, the classical limit of $\mathcal{J}^{\rho \nu}$ can be identified to $i \mathbf{J}^{\rho \nu}$, where $\mathbf{J}^{\rho \nu}$ denote components of the total angular momentum. In this limit we can express (3.5) as

$$
S_{\mathrm{gr}}^{(1)}(\varepsilon, k)=i \sum_{a=1}^{n} \frac{\varepsilon_{\mu \nu} p_{(a)}^{\mu} k_{\rho}}{p_{(a)} \cdot k} \mathbf{J}_{(a)}^{\rho \nu} .
$$

We shall now try to estimate the relative importance of the two subleading terms in (3.3) in the classical limit. In this limit the momenta $p_{(a)}$ and the angular momenta $\mathbf{J}_{(a)}$ become large. Let us take $p_{(a)} \sim \mu$ and $\mathbf{J}_{(a)} \sim \mu \lambda$ for some large $\mu, \lambda$. Here $\mu$ is the typical mass / energy of the macroscopic objects and $\lambda$ is the typical length scale of the problem, measured in Planck units. $\lambda^{-1}$ will provide an effective upper cut-off on the frequency of soft radiation, and hence, by definition, soft gravitons will carry energies small compared to $\lambda^{-1}$. So we take $k \sim \lambda^{-1} \tau$ where $\tau$ is the small number which controls the soft expansion. Then we have

$$
\begin{aligned}
S_{\mathrm{gr}}^{(0)}(\varepsilon, k) & \sim \mu \lambda \tau^{-1}, \quad S_{\mathrm{gr}}^{(1)}(\varepsilon, k) \sim \mu \lambda, \quad \mathcal{M}\left(p ; \varepsilon_{1}, k_{1}, \varepsilon_{2}, k_{2}\right) \sim \mu^{2}, \\
\left\{p_{(a)} .\left(k_{r}+k_{u}\right)\right\}^{-1} & \sim \mu^{-1} \lambda \tau^{-1} .
\end{aligned}
$$


It follows from this that for finite number of soft gravitons, the three terms in (3.3) are of order $\mu^{M} \lambda^{M} \tau^{-M}, \mu^{M} \lambda^{M} \tau^{-M+1}$ and $\mu^{M-1} \lambda^{M-1} \tau^{-M+1}$ respectively. Therefore in the classical limit $\mu, \lambda \rightarrow \infty$, we can drop the third term compared to the other two. However since we shall also take $M$ to be large in this limit, we need to be more careful in our estimate. We shall proceed for now by dropping the third term, but later confirm that it is indeed small compared to the second term for the value of $M$ where the probability distribution peaks.

With this understanding we replace (3.3) by

$$
\left\{\prod_{r=1}^{M} S_{\mathrm{gr}}^{(0)}\left(\varepsilon_{r}, k_{r}\right)\right\}+\sum_{s=1}^{M}\left\{\prod_{\substack{r=1 \\ r \neq s}}^{M} S_{\mathrm{gr}}^{(0)}\left(\varepsilon_{r}, k_{r}\right)\right\} S_{\mathrm{gr}}^{(1)}\left(\varepsilon_{s}, k_{s}\right) .
$$

Since $S_{\mathrm{gr}}^{(0)}$ and $S_{\mathrm{gr}}^{(1)}$ are both multiplicative $c$-numbers, to this order (3.8) can also be written as ${ }^{6}$

$$
\prod_{r=1}^{M} S_{\mathrm{gr}}\left(\varepsilon_{r}, k_{r}\right), \quad S_{\mathrm{gr}}\left(\varepsilon_{r}, k_{r}\right) \equiv\left\{S_{\mathrm{gr}}^{(0)}\left(\varepsilon_{r}, k_{r}\right)+S_{\mathrm{gr}}^{(1)}\left(\varepsilon_{r}, k_{r}\right)\right\} .
$$

Going from (3.8) to (3.9) requires some discussion. For finite $M$, these two expressions are clearly equal up to the subleading order. However in our analysis the number of soft gravitons will be large and for this the two expressions are not equal. Therefore we need to decide on which one is the correct expression for large $M$. This can be done by going back to the analysis of [78] and repeating the analysis dropping all terms that vanish in the classical limit, i.e. are suppressed by powers of $\mu$ in the denominator. This includes the 'contact terms' leading to $\mathcal{M}$ and also terms that involve commutators of $\partial / \partial p_{\mu}$ and $p_{\nu}$ since this reduces the number of powers of $\mu$. In this case it becomes clear that the soft factor is given by the product of $r$ independent factors, one for each soft graviton, and is given by (3.9). Note that (3.9) is also the form that we shall get if we take the soft limit consecutively using single soft theorem multiple times. Before taking the classical limit the result of consecutive soft limit is not symmetric under the exchange of the soft gravitons, i.e. it preserves the memory of which one was taken to be soft first, but in the classical limit the symmetry is restored since $S_{\mathrm{gr}}^{(0)}$ and $S_{\mathrm{gr}}^{(1)}$ commute. This also provides further support to the fact that (3.9) is the correct form of the subleading multiple soft theorem for large number of soft gravitons.

It now follows that to subleading order in the expansion parameter $\omega a$, the number of soft gravitons in given range of energy and solid angle, and the energy carried by them, are given respectively by $(2.6)$ and $(2.7)$ with $S_{\mathrm{em}}^{(0)}(\varepsilon, k)$ replaced

\footnotetext{
${ }^{6}$ Note that (3.9) contains subsubleading contributions that may be written schematically, by dropping the arguments, as $\left(S_{\mathrm{gr}}^{(0)}\right)^{M-2}\left(S_{\mathrm{gr}}^{(1)}\right)^{2}$. One could ask in what sense this is more important than the subsubleading contributions of the form $\left(S_{\mathrm{gr}}^{(0)}\right)^{M-1} S_{\mathrm{gr}}^{(2)}$ where $S_{\mathrm{gr}}^{(2)}$ is the subsubleading contribution to the single soft graviton theorem. To this end note that there are $\left(\begin{array}{c}M \\ 2\end{array}\right)$ terms of the form $\left(S_{\mathrm{gr}}^{(0)}\right)^{M-2}\left(S_{\mathrm{gr}}^{(1)}\right)^{2}$ and $M$ terms of the form $\left(S_{\mathrm{gr}}^{(0)}\right)^{M-1} S_{\mathrm{gr}}^{(2)}$. Therefore while for finite $M$ they are of the same order, for large $M$ the $\left(S_{\mathrm{gr}}^{(0)}\right)^{M-2}\left(S_{\mathrm{gr}}^{(1)}\right)^{2}$ contributions dominate.
} 
by $S_{\mathrm{gr}}(\varepsilon, k) \equiv S_{\mathrm{gr}}^{(0)}(\varepsilon, k)+S_{\mathrm{gr}}^{(1)}(\varepsilon, k)$ :

$$
\begin{aligned}
& N=\frac{1}{2^{D} \pi^{D-1}}\left|S_{\mathrm{gr}}(\varepsilon, k)\right|^{2} \omega^{D-2} \Delta \Omega \delta, \\
& E=N \omega=\frac{1}{2^{D} \pi^{D-1}}\left|S_{\mathrm{gr}}(\varepsilon, k)\right|^{2} \omega^{D-1} \Delta \Omega \delta .
\end{aligned}
$$

However for the validity of this equation we need to satisfy several conditions:

- The number of soft gravitons emitted in a given bin must be large.

- The third term in (3.3), encoding the effect of the contact term $\mathcal{M}$, must be small compared to the second term.

- The total energy / angular momentum carried away by the radiation must be small compared to the energy and the angular momentum carried by the macroscopic objects.

We shall now describe under what condition this holds. In particular we shall see that the last two conditions lead to identical constraints.

Using the scaling described in (3.7) and the paragraph above it, the number of soft gravitons emitted in a given bin given by (3.10) can be estimated as:

$$
N \sim \mu^{2} \lambda^{-(D-4)} \tau^{D-4} \Delta \Omega \delta .
$$

For fixed $\tau, \delta$ and $\Omega$, whether this is large or not depends on the relative magnitude of $\mu$ and $\lambda$. Now in $D$ space-time dimensions, the mass $m$ of a black hole scales with its Schwarzschild radius $a$ as

$$
a \sim m^{1 /(D-3)}
$$

Since $\mu$ and $\lambda$ denote the typical mass and length scales of the classical object, it is natural to introduce a parameter $\sigma$ via the relation

$$
\lambda \sim \mu^{1 /(D-3)} \sigma
$$

where $\sigma \gtrsim 1$ measures how large the typical length scale is compared to the Schwarzschild radii $\sim \mu^{1 /(D-3)}$ of the macroscopic objects involved in the scattering. In that case (3.12) may be expressed as

$$
N \sim \mu^{(D-2) /(D-3)} \tau^{D-4} \sigma^{-(D-4)} \Delta \Omega \delta .
$$

Even though $\tau$ is a small number and $\sigma \gtrsim 1$, we see that by taking $\mu$ to be sufficiently large we can ensure that the number of gravitons emitted in a given bin is large.

Let us now recall that for our expansion to be valid, we also need to ensure that the ratio of the third term to the second term in (3.3) must be small. Using (3.7) we find that for large $M$ two subleading terms in (3.3) are of order

$$
M \mu^{M} \lambda^{M} \tau^{-M+1}, \quad M^{2} \mu^{M-1} \lambda^{M-1} \tau^{-M+1},
$$


respectively. Here the factors $M$ and $M^{2}$ arise from the fact that there are $M$ terms in the sum in the second term and $M(M-1) / 2$ terms in the sum in the third term. Therefore the ratio of the third term to the second term is given by

$$
M \mu^{-1} \lambda^{-1} \text {. }
$$

Since the third term in (3.3) can receive contribution not just from the pair of gravitons in the same bin, but also from pair of gravitons in different bins, it is appropriate to take for $M$ in (3.17) the total number of gravitons emitted during the process. This can be estimated by setting $\tau, \Delta \Omega, \delta \sim 1$ on the right hand side of (3.12), reflecting that the inverse of the typical length scale $\lambda$ also sets the upper cut-off on $\omega$ for the radiation emitted during the scattering. This gives

$$
M \sim \mu^{2} \lambda^{-(D-4)}
$$

Therefore (3.17) reduces to

$$
\mu \lambda^{-(D-3)} \sim \sigma^{-(D-3)} .
$$

This shows that if we can keep $\sigma$ large then the ratio given in (3.17) is small and our approximation is valid. When the macroscopic objects have masses of the same order, then this can be achieved by taking the typical length scale, e.g. the impact parameter, to be large compared to the Schwarzschild radii of each object. The other possibility is to take the probe limit that will be discussed shortly.

We shall now show that this condition is equivalent to keeping small the ratio of the total energy carried by the gravitons and the typical mass of the macroscopic objects involved in the scattering. The latter is of order $\mu$ whereas the former can be estimated by multiplying (3.12) by $\omega \sim \lambda^{-1} \tau$ to get the energy of the gravitons in a given bin, and then by setting $\Delta \Omega \sim 1, \delta \sim 1, \tau \sim 1$. This gives the ratio to be of order

$$
\left[\left\{\mu^{-1}\right\}\left\{\lambda^{-1} \tau\right\}\left\{\mu^{2} \lambda^{-(D-4)} \tau^{D-4}\right\}\right]_{\tau=1} \sim \sigma^{-(D-3)} .
$$

This is identical to (3.19) and can be kept small by taking $\sigma$ to be large.

Similar analysis can be done for the ratio of the total angular momentum carried away by the radiation to the typical angular momenta of the macroscopic objects. The former is of order $M$ given in (3.18) whereas the latter is of order $\mu \lambda$. Therefore the ratio of the two is given by

$$
\mu \lambda^{-(D-3)} \sim \sigma^{-(D-3)} .
$$

Therefore this remains small for large $\sigma$.

It is not difficult to trace why keeping the term proportional to $\mathcal{M}$ in (3.3) small also ensures that the angular momentum and energy carried away by the radiation remains small compared to the angular momenta and energies of the macroscopic objects. The contributions to $S_{\mathrm{gr}}^{(0)}$ and $S_{\mathrm{gr}}^{(1)}$ from a given external state are proportional respectively to the momentum and the angular momentum of the state. Now in writing the expressions for $S_{\mathrm{gr}}^{(0)}$ and $S_{\mathrm{gr}}^{(1)}$ we have taken the sum over $a$ to run only over the macroscopic objects and not included the contributions from the radiation, but the contact interaction $\mathcal{M}$ includes these contributions. Therefore keeping the effect of $\mathcal{M}$ small requires that the total energy and 
angular momentum carried away by the radiation remain small compared to the energies and angular momenta of the macroscopic objects contributing to (3.2), (3.6).

Let us now describe the other way of keeping $\sigma$ large — namely by taking the probe limit. For simplicity we shall consider the probe limit of the $2 \rightarrow 2$ scattering although the analysis can be easily generalized to multi-body scattering. We shall use a somewhat more general kinematics for finite energy external particles than the one discussed in (2.8), (2.9). Instead of assuming that the masses of the probe and the scatterer remain unchanged during the scattering, we allow them to change. This will include the situation e.g. where a part of the probe is stripped off and absorbed by the scatterer during the scattering process. The new kinematics takes the form

$$
\begin{array}{ll}
p_{(1)}=\left(p_{(1)}^{0}, \vec{p}_{(1)}\right), & p_{(2)}=\left(p_{(2)}^{0}, \vec{p}_{(2)}\right), \\
p_{(3)}=\left(M_{0}, \overrightarrow{0}\right), & p_{(4)}=\left(-M_{0}-p_{(1)}^{0}-p_{(2)}^{0},-\vec{p}\right),
\end{array}
$$

where

$$
\vec{p} \equiv \vec{p}_{(1)}+\vec{p}_{(2)}, \quad p_{(1)}^{0}=\sqrt{\vec{p}_{(1)}^{2}+m_{(1)}^{2}}, \quad p_{(2)}^{0}=-\sqrt{\vec{p}_{(2)}^{2}+m_{(2)}^{2}} .
$$

This parametrization manifestly implements the conservation of all components of momenta. We now take the $M_{0} \rightarrow \infty$ limit keeping $p_{(a)}$ for $a=1,2$ fixed. In this limit (3.2) reduces to

$$
S_{\mathrm{gr}}^{(0)}=\sum_{a=1}^{2}\left[\frac{\varepsilon_{\mu \nu} p_{(a)}^{\mu} p_{(a)}^{\nu}}{p_{(a)} \cdot k}+\varepsilon_{00} \frac{p_{(a)} \cdot k}{\left(k^{0}\right)^{2}}+2 \varepsilon_{0 \nu} \frac{p_{(a)}^{\nu}}{k^{0}}\right] .
$$

For analyzing the subleading soft factor $S_{\mathrm{gr}}^{(1)}$, we shall choose the origin of space coordinate to be at the center of mass of the scatterer before scattering - defined such that the $0 i$ components of its angular momentum vanish. In that case we can label $\mathbf{J}_{(a)}^{\mu \nu}$ of the four external states as follows:

$$
\begin{array}{ll}
\mathbf{J}_{(1)}^{\mu \nu}=\mathbf{j}_{(1)}^{\mu \nu}, & \mathbf{J}_{(2)}^{\mu \nu}=\mathbf{j}_{(2)}^{\mu \nu}, \\
\mathbf{J}_{(3)}^{i j}=\mathbf{J}^{i j}, & \mathbf{J}_{(3)}^{0 i}=0, \quad \text { for } \quad 1 \leq i, j \leq D-1, \quad \mathbf{J}_{(4)}^{\mu \nu}=-\mathbf{J}_{(1)}^{\mu \nu}-\mathbf{J}_{(2)}^{\mu \nu}-\mathbf{J}_{(3)}^{\mu \nu} .
\end{array}
$$

Here $\mathbf{J}^{i j}$ is a classical angular momentum carried by the scatterer before the scattering. We now take the limit $M_{0} \rightarrow \infty$ keeping the ratio $\mathbf{J}^{i j} / M_{0}$ fixed. In this limit the subleading soft factor reduces to

$$
S_{\mathrm{gr}}^{(1)}=i \sum_{a=1}^{2}\left[\left\{\frac{\varepsilon_{\mu \nu} p_{(a)}^{\mu} k_{\rho}}{p_{(a)} \cdot k}+\frac{\varepsilon_{\nu 0} k_{\rho}}{k^{0}}\right\} \mathbf{j}_{(a)}^{\rho \nu}+\frac{\mathbf{J}^{j i}}{M_{0}}\left\{\frac{\varepsilon_{i 0} k_{j} p_{(a)} \cdot k}{\left(k^{0}\right)^{2}}+\frac{\varepsilon_{i \nu} p_{(a)}^{\nu} k_{j}}{k^{0}}\right\}\right] .
$$

It is easy to see that the right hand side of (3.24), (3.26) are invariant under the gauge transformation

$$
\varepsilon_{\mu \nu} \rightarrow \varepsilon_{\mu \nu}+\xi_{\mu} k_{\nu}+\xi_{\nu} k_{\mu},
$$

for any vector $\xi$.

We now need to check that in this limit the contribution of the third term in (3.3) is suppressed compared to the second term. We have already seen that when the impact parameter is large compared to the Schwarzschild radius of the scatterer then this is true 
even without the probe limit, so let us focus on the case where the impact parameter is of the same order as the Schwarzschild radius $a$ of the scatterer. We also assume that the probe moves with speed of order unity and that $m_{(1)} \sim m_{(2)} \sim m$. In this case we have

$$
M_{0} \sim a^{D-3}, \quad \mathbf{J} \sim M_{0} a \sim a^{D-2}, \quad p_{(a)}^{\mu} \sim m, \quad \mathbf{j}_{(a)} \sim m a \quad \text { for } \quad a=1,2,
$$

and the upper cut-off on $\omega$ is of order $a^{-1}$. Therefore we have

$$
\begin{array}{rlrl}
S^{(0)} & \sim m / \omega, & S^{(1)} & \sim(m / \omega)(\omega a), \\
\mathcal{M}\left(p_{(a)} ; \varepsilon_{1}, k_{1}, \varepsilon_{2}, k_{2}\right) & \sim m^{2}, \quad\left\{p_{(a)} .\left(k_{r}+k_{u}\right)\right\}^{-1} & \sim(m \omega)^{-1}, \quad \text { for } a=1,2, \\
\sum_{a=3}^{4}\left\{p_{(a)} .\left(k_{r}+k_{u}\right)\right\}^{-1} \mathcal{M}\left(p_{(a)} ; \varepsilon_{1}, k_{1}, \varepsilon_{2}, k_{2}\right) & \sim m \omega^{-1} .
\end{array}
$$

Comparing this with (3.7) we see that the scalings in (3.29) and (3.7) are identical if we take $\mu \sim m, \lambda \sim a$ and $\tau \sim \omega a$. This also gives $\sigma \equiv \lambda \mu^{-1 /(D-3)} \sim a m^{-1 /(D-3)} \sim$ $\left(M_{0} / m\right)^{1 /(D-3)}$. Therefore for $M_{0} \gg m, \sigma$ is large. This ensures that the contribution to the soft theorem from the terms proportional to $\mathcal{M}$ is supressed. Equivalently, the energy and angular momentum carried away by radiation remains small compared to the energy and angular momentum carried by the macroscopic objects involved in the scattering. In particular the total amount of energy radiated is of order

$$
\left[\left|S_{\mathrm{gr}}^{(0)}(\varepsilon, k)+S_{\mathrm{gr}}^{(1)}(\varepsilon, k)\right|^{2} \omega^{D-1}\right]_{\omega=a^{-1}} \sim m^{2} / M_{0} .
$$

This is small compared to $m$ for $m \ll M_{0}$.

We see from (3.29) that the subleading contribution is suppressed compared to the leading term by order $\omega a$. Therefore $\omega a$ is the soft expansion parameter. This is not surprising since in this case the Schwarzschild radius of the scatterer determines the typical length scale of the problem.

We end with some observations on the final result:

1. For plane polarized gravitational waves $S_{\mathrm{gr}}^{(0)}$ is real while $S_{\mathrm{gr}}^{(1)}$ is purely imaginary. Therefore the contribution of $S_{\mathrm{gr}}^{(1)}$ to the scattering cross-section vanishes to subleading order. However if we use circularly polarized gravitational wave then $\varepsilon_{\mu \nu}$ is complex and as a result both $S_{\mathrm{gr}}^{(0)}$ and $S_{\mathrm{gr}}^{(1)}$ are complex. In this case we expect $S_{\mathrm{gr}}^{(1)}$ to contribute to the cross section at subleading order. Related comments can be found in $[86]$.

2. As mentioned below (2.6), even though we have presented our analysis as if it relies on the computation of the amplitude with $N$ soft gravitons in a given momentum bin and no other particles in the final state other than the probe and the scatterer, our result is valid more generally. Production of other soft gravitons in other momentum bins will give an additional soft multiplicative factor in the amplitude (2.3), but will not affect $N$ dependence and the analysis leading to (3.10) remains valid. Inclusion of 'hard gravitons' in the final state, carrying momentum of order $1 / a$, may not be 
represented as a multiplicative factor, and may modify $\mathbf{M}$ appearing in (2.3) in a nontrivial manner, but it still will not change the $N$ dependence of (2.3). Therefore (3.10) still remains valid.

3. Under a shift in the origin of space-time by $c^{\mu}, \mathbf{J}^{\rho \nu}$ gets shifted by $c^{\rho} p_{(a)}^{\nu}-c^{\nu} p_{(a)}^{\rho}$. This changes $S_{\mathrm{gr}}^{(1)}$ given in (3.6) to $i c . k S_{\mathrm{gr}}^{(0)}$. Therefore to subleading order

$$
S_{\mathrm{gr}}^{(0)}+S_{\mathrm{gr}}^{(1)} \rightarrow e^{i c . k}\left(S_{\mathrm{gr}}^{(0)}+S_{\mathrm{gr}}^{(1)}\right) .
$$

Since this is just an overall phase, this does not affect any physical quantities.

4. (3.2) and (3.6) (and (3.24) and (3.26)) do not depend on the details of the theory or the macroscopic objects involved in the scattering except their momenta and angular momenta. However the subsubleading contribution will depend on the details of the macroscopic objects (and the theory) via the non-universal terms that appear in the subsubleading soft graviton theorem. This will be discussed in section 6 .

5. If the scattering configuration is such that the angular momenta $\mathbf{J}_{(a)}$ appearing in (3.6) receive dominant contribution from the orbital angular momentum, we can express $\mathbf{J}_{(a)}$ in terms of the classical trajectories of the incoming and outgoing macroscopic objects as follows. Let the trajectory of the center of momentum of the $a$-th object be given by

$$
x_{(a)}^{\mu}=c_{(a)}^{\mu}+m_{(a)}^{-1} p_{(a)}^{\mu} \tau_{a},
$$

where $\tau_{a}$ are the proper time labelling the trajectories. Then $\mathbf{J}_{(a)}^{\mu \nu}$, dominated by the orbital angular momenta, are given by

$$
\mathbf{J}_{(a)}^{\mu \nu}=c_{(a)}^{\mu} p_{(a)}^{\nu}-c_{(a)}^{\nu} p_{(a)}^{\mu} .
$$

6. In arriving at the expression (3.26) for $S_{\mathrm{gr}}^{(1)}$ we have already fixed the origin of the spatial coordinate system to be at the initial position of the scatterer. But we still have the freedom of shifting the origin of the time coordinate. This shifts $c_{(a)}^{0}$ in (3.32), (3.33) for the probe by some constant $c^{0}$. It can be checked that under such a shift $S_{\mathrm{gr}} \equiv S_{\mathrm{gr}}^{(0)}+S_{\mathrm{gr}}^{(1)}$ given in (3.24), (3.26) picks up an overall phase $\exp \left[-i c^{0} k^{0}\right]$ and as a result (3.10), (3.11) remains unchanged.

Note that by appropriately choosing the origin of the time coordinate we can set $c_{(1)}^{0}$ to 0 . But we do not have the freedom of also setting $c_{(2)}^{0}$ to 0 . Therefore $S_{\mathrm{gr}}^{(1)}$ depends non-trivially on the time delay encoded in $c_{(2)}^{0}$.

7. The soft factors given in (3.24) and (3.26) are gauge invariant without using any momentum or angular momentum conservation laws. We could in fact replace the soft factors (3.2) and (3.6) before taking the probe limit by those in (3.24) and (3.26) respectively provided the sum over $a$ runs over all macroscopic objects, since the contribution from the additional terms would vanish after using momentum and angular momentum conservation. The resulting soft factor is not manifestly Lorentz 
covariant, but is manifestly gauge invariant before using momentum and angular momentum conservation laws. Once we use momentum and angular momentum conservation laws, then both gauge and Lorentz invariance are manifest.

8. The soft factors (3.24) and (3.26) vanish in the $\vec{p} \rightarrow 0$ limit, i.e. in the limit in which the probe is at rest relative to the scatterer. However if we keep $\vec{p} / m$ small but fixed as we take the classical limit, our results continue to hold and give the angular power spectrum of gravitational radiation for non-relativistic scattering.

\section{Sum over polarizations for probe scattering}

Given the angular power spectrum for given polarization, we can sum over polarizations to get the total angular power spectrum. For this we can use the basis of plane polarized waves. Since we have argued that in this basis the power spectrum is independent of the subleading correction to subleading order, we can just focus on the contribution from the leading soft factor. In the probe approximation this is given in (3.24).

Now the last two terms in (3.24) can be thought of as the effect of radiation from the scatterer since they come from the $a=3,4$ terms in the sum in (3.2) - although there is really no gauge invariant way of isolating the radiation from the scatterer and the radiation from the probe. By choosing the transverse gauge in which $\varepsilon_{i 0}$ and $\varepsilon_{00}$ vanish, we can make the last two terms in (3.24) vanish. Therefore it would seem that after we sum over polarizations the effect of radiation from the scatterer disappears. However we shall now see that this is not so. For this we express (3.24) as

$$
S^{(0)}(\varepsilon, k)=\varepsilon_{\mu \nu} \Sigma^{\mu \nu}(k),
$$

where

$$
\Sigma^{\mu \nu}=\sum_{a=1}^{2}\left[\frac{p_{(a)}^{\mu} p_{(a)}^{\nu}}{p_{(a)} \cdot k}+\delta_{0}^{\mu} \delta_{0}^{\nu} \frac{p_{(a)} \cdot k}{\left(k^{0}\right)^{2}}+\left\{\delta_{0}^{\mu} p_{(a)}^{\nu}+\delta_{0}^{\nu} p_{(a)}^{\mu}\right\} \frac{1}{k^{0}}\right] .
$$

Gauge invariance now translates to

$$
k_{\mu} \Sigma^{\mu \nu}(k)=0, \quad k_{\nu} \Sigma^{\mu \nu}(k)=0 .
$$

Let $\varepsilon_{\mu \nu}^{(\alpha)}$ for $1 \leq \alpha \leq D(D-3) / 2$ denote the independent polarization tensors in some gauge. Then the total angular power spectrum is proportional to

$$
\sum_{\alpha=1}^{D(D-3) / 2}\left|S^{(0)}\left(\varepsilon^{(\alpha)}, k\right)\right|^{2}=\sum_{\alpha=1}^{D(D-3) / 2} \varepsilon_{\mu \nu}^{(\alpha) *} \varepsilon_{\rho \sigma}^{(\alpha)} \Sigma^{\mu \nu}(k) \Sigma^{\rho \sigma(k)} .
$$

Now we have

$$
\begin{aligned}
\sum_{\alpha=1}^{D(D-3) / 2} \varepsilon_{\mu \nu}^{(\alpha) *} \varepsilon_{\rho \sigma}^{(\alpha)}= & \frac{1}{2}\left(\eta_{\mu \rho} \eta_{\nu \sigma}+\eta_{\mu \sigma} \eta_{\nu \rho}\right)-\frac{1}{D-2} \eta_{\mu \nu} \eta_{\rho \sigma} \\
& + \text { terms proportional to } k_{\mu}, k_{\nu}, k_{\rho} \text { or } k_{\sigma}
\end{aligned}
$$


Substituting this into (4.4), and using (4.3) we get

$$
\sum_{\alpha=1}^{D(D-3) / 2}\left|S^{(0)}\left(\varepsilon^{(\alpha)}, k\right)\right|^{2}=\Sigma^{\mu \nu}(k) \Sigma_{\mu \nu}(k)-\frac{1}{D-2} \Sigma_{\mu}^{\mu}(k) \Sigma_{\nu}^{\nu}(k) .
$$

It is easy to see from this that the last two terms in (4.2) does contribute to this.

If we had chosen transverse polarizations from the beginning then we could have dropped the last two terms in (4.2). But the resulting expression will not satisfy (4.3) and therefore the terms proportional to $k$ in (4.5) will now contribute. The final result of course will remain unchanged.

We can arrive at expression (4.6) by starting with (3.2) and performing the sum over polarizations before taking the $M_{0} \rightarrow \infty$ limit. This will give [85]

$$
\sum_{\alpha=1}^{D(D-3) / 2}\left|S^{(0)}\left(\varepsilon^{(\alpha)}, k\right)\right|^{2}=\sum_{a, b=1}^{4} \frac{1}{p_{(a)} \cdot k p_{(b)} \cdot k}\left[\left(p_{(a)} \cdot p_{(b)}\right)^{2}-\frac{1}{D-2} p_{(a)}^{2} p_{(b)}^{2}\right]
$$

This is a special case of the general formula derived in [97]. It is easy to verify that $M_{0} \rightarrow \infty$ limit on (4.7) reproduces (4.6). The contribution to (4.6) from the last two terms in (4.2) arise from the terms in (4.7) with either $a$ or $b$ or both taking values 3 or 4 .

It follows from (4.5) that given the radiative part of the soft graviton field $h_{\mu \nu}$, its projection to a given polarization tensor $\varepsilon^{\mu \nu}$ can be obtained by contracting $\varepsilon_{\mu \nu}$ to $h_{\rho \sigma}$ by the inverse of the matrix appearing in (4.5):

$$
\varepsilon_{\mu \nu}\left\{\frac{1}{2}\left(\eta^{\mu \rho} \eta^{\nu \sigma}+\eta^{\mu \sigma} \eta^{\nu \rho}\right)-\frac{1}{2} \eta^{\mu \nu} \eta^{\rho \sigma}\right\} h_{\rho \sigma}
$$

\section{Plunge}

We can also consider the case where a pair of macroscopic objects fuse into each other and form a third object. As in section 3, we can keep the energy carried by gravitational radiation small compared to the initial and the final energies of the macroscopic objects by making the effective impact parameter large, e.g. by taking the sizes of the objects to be large compared to their Schwarzschiid radii so that their centers always remain far apart. In this case the soft factor is given by (3.2), (3.6) with $n=3$, representing the two initial objects and one final object.

Alternatively we can consider the probe approximation where the probe fuses to the scatterer and produces a third object with slightly more mass than the scatterer. This process will have three external finite energy particles, whose momenta and angular momenta can be taken as

$$
\begin{aligned}
& p_{(1)}=\left(p^{0}, \vec{p}\right), \quad p_{(2)}=\left(M_{0}, \overrightarrow{0}\right), \quad p_{(3)}=\left(-M_{0}-p^{0},-\vec{p}\right), \\
& \mathbf{J}_{(1)}^{\mu \nu}=\mathbf{j}^{\mu \nu}, \quad \mathbf{J}_{(2)}^{i j}=\mathbf{J}^{i j}, \quad \mathbf{J}_{(2)}^{0 i}=0, \quad \text { for } \quad 1 \leq i, j \leq D-1, \quad \mathbf{J}_{(3)}^{\mu \nu}=-\mathbf{J}_{(1)}^{\mu \nu}-\mathbf{J}_{(2)}^{\mu \nu}
\end{aligned}
$$


We can now compute soft graviton radiation from this process using the soft theorem. The soft factor up to subleading order is given by

$$
S_{\mathrm{gr}}(k)=\sum_{a=1}^{3} \frac{\varepsilon_{\mu \nu} p_{(a)}^{\mu} p_{(a)}^{\nu}}{p_{(a)} \cdot k}+i \sum_{a=1}^{3} \frac{\varepsilon_{\mu \nu} p_{(a)}^{\mu} k_{\rho}}{p_{(a)} \cdot k} \mathbf{J}_{(a)}^{\rho \nu} .
$$

Taking $M_{0} \rightarrow \infty$ limit with $\mathbf{J} / M_{0}$ fixed gives

$$
\begin{aligned}
S_{\mathrm{gr}}(k)= & \left\{\frac{\varepsilon_{\mu \nu} p^{\mu} p^{\nu}}{p . k}+\varepsilon_{00} \frac{p . k}{\left(k^{0}\right)^{2}}+2 \varepsilon_{0 \nu} \frac{p^{\nu}}{k^{0}}\right\} \\
& +i\left\{\left(\frac{\varepsilon_{\mu \nu} p^{\mu} k_{\rho}}{p . k}+\frac{\varepsilon_{0 \nu} k_{\rho}}{k^{0}}\right) \mathbf{j}^{\rho \nu}+\frac{J^{j i}}{M_{0}}\left(\frac{\varepsilon_{0 i} k_{j}}{\left(k^{0}\right)^{2}} p \cdot k+\varepsilon_{\sigma i} p^{\sigma} \frac{k_{j}}{k^{0}}\right)\right\} .
\end{aligned}
$$

This formula can also be regarded as a special case of (1.2), (1.3) in which we take the limit $p_{(2)} \rightarrow 0, \mathbf{j}_{(2)} \rightarrow 0$. The angular power spectrum of soft radiation can now be found from (3.11).

Again in the probe limit we can estimate the total radiated energy to be of order $m^{2} / M_{0}$. Since this is small compared to $m$ in the $M_{0} \gg m$ limit, we expect our approximation to be valid.

The reverse of this process is decay, where a macroscopic object splits apart into two objects due to some internal dynamics. The corresponding formulæ are the same as the ones given in this section, but the signs of the $p_{(a)}$ 's will be reversed.

\section{$6 \quad$ Universal parts of higher order non-universal results}

In this section we shall explore the possibility of extracting universal parts of higher order soft theorems, both for electromagnetism and for gravity.

\subsection{Subleading soft photon theorem}

Unlike gravitational theories, there is no universal subleading soft photon theorem - the $S_{\mathrm{em}}^{(1)}$ factor depends on the theory, and can also depend on which external particle we consider. This can be traced to the non-minimal coupling of the soft photon to the external particles via the field strength $F_{\mu \nu}$ - since this has one derivative, it produces a subleading correction. Nevertheless part of the expression for $S_{\mathrm{em}}^{(1)}$ is independent of the details of the theory. This takes the form [33]

$$
S_{\mathrm{em}}^{(1)}(\varepsilon, k)=\sum_{a=1}^{n} q_{(a)} \frac{\varepsilon_{\nu} k_{\rho} \mathcal{J}^{\rho \nu}}{p_{(a)} \cdot k}=i \sum_{a=1}^{n} q_{(a)} \frac{\varepsilon_{\nu} k_{\rho}}{p_{(a)} \cdot k} \mathbf{J}_{(a)}^{\rho \nu},
$$

in the same convention as was used in (3.5), (3.6). A general derivation of this formula for any external states in any theory can be given following the procedure described in [77]. For large impact parameter $b$ the angular momenta of the macroscopic objects are dominated by the orbital part and have the form given in (3.33). If $m$ denotes the typical mass of the macroscopic objects involved in the scattering, then $\mathbf{J}_{(a)}^{\rho \nu} \sim m b$. On the other hand, the non-universal corrections to the soft photon theorem are expected to be proportional 
to $m a$ where $a$ is the typical size of the objects. Therefore for $b \gg a$ the universal part of $S_{\mathrm{em}}^{(1)}$ dominates and we can trust (6.1).

In the probe limit we use the kinematics given in (2.8) and take the limit $M_{0} \rightarrow \infty$. This gives

$$
S_{\mathrm{em}}^{(1)}(\varepsilon, k)=i \sum_{a=1}^{2} q_{(a)} \frac{\varepsilon_{\nu} k_{\rho}}{p_{(a)} \cdot k} \mathbf{j}_{(a)}^{\rho \nu} .
$$

Again, for large impact parameter $b$ the angular momenta of the macroscopic objects are dominated by the orbital part and have the form given in (3.33). Substituting (3.33) into (6.2), using $q_{(a)}=(-1)^{a-1} q$ for $a=1,2$, and adding the result to (2.11), we get the total soft factor for photons to subleading order

$$
S_{\mathrm{em}}(\varepsilon, k)=q \sum_{a=1}^{2}(-1)^{a-1} \frac{\varepsilon \cdot p_{(a)}}{k \cdot p_{(a)}}+i q \sum_{a=1}^{2}(-1)^{a} \frac{1}{p_{(a)} \cdot k}\left\{c_{(a)} \cdot k p_{(a)} \cdot \varepsilon-c_{(a)} \cdot \varepsilon p_{(a)} \cdot k\right\}
$$

Since $c_{(a)} \sim b$, we see that the subleading contribution is of order $q b$. We expect this to dominate the non-universal corrections to $S_{\mathrm{em}}^{(1)}$ when the impact parameter $b$ is large compared to the sizes $a$ of the probe and the scatterer. In this case the non-universal terms are expected to be of order $q a$, which is small compared to the subleading contribution of order $q b$ given above.

The results described in (6.2) were given for $M_{0} \rightarrow \infty$ limit at fixed $Q$. If we take the $M_{0} \rightarrow \infty$ limit at fixed $Q / M_{0}$ and $\mathbf{J}^{i j} / M_{0}$, we have additional subleading contributions:

$$
\Delta S_{\mathrm{em}}^{(1)}=i \frac{Q}{M_{0}} \frac{\mathbf{J}^{i j}}{M_{0}} \varepsilon_{j} k_{i} \frac{\vec{p} \cdot \vec{k}}{\left(k^{0}\right)^{2}}+i \frac{Q}{M_{0}} \frac{1}{k^{0}} \varepsilon_{\nu} k_{\rho}\left(\mathbf{j}_{(1)}^{\rho \nu}+\mathbf{j}_{(2)}^{\rho \nu}\right)
$$

\subsection{Subsubleading soft graviton theorem}

We can try to carry out a similar analysis for subsubleading soft theorem for gravity. In our convention the universal part of the soft factor to this order may be written as $[13,14$, $31,32,77]$

$$
S_{\mathrm{gr}}^{(2)}=-\frac{1}{2} \sum_{a=1}^{n}\left(p_{(a)} \cdot k\right)^{-1} \varepsilon_{\mu \rho} k_{\nu} k_{\sigma} \mathbf{J}_{(a)}^{\mu \nu} \mathbf{J}_{(a)}^{\rho \sigma} .
$$

For large impact parameter $b$, the orbital angular momenta are of order $m b$ where $m$ is the typical energy of the macroscopic objects, and the spin angular momenta are of order ma where $a$ is the typical size of these objects. Therefore the three kinds of terms arising out of the expansion of (6.5), the square of the orbital angular momentum, the square of the spin angular momentum and the cross term, are respectively of order $m \omega b^{2}, m \omega a^{2}$ and $m \omega a b$. The non-universal corrections to (6.5) are expected to be of order $m \omega a^{2}$ - of the same order as the square of the spin angular momentum term. Therefore in the $b \gg a$ limit, it is appropriate to keep the terms involving square of the orbital angular momenta and the cross term between the orbital and the spin angular momenta, but not the term proportional to the square of the spin angular momenta. 
If we consider $2 \rightarrow 2$ scattering and take the probe limit $M_{0} \rightarrow \infty$ with the kinematics described in (3.22), (3.23), then (6.5) becomes

$$
\begin{aligned}
S_{\mathrm{gr}}^{(2)}= & -\frac{1}{2} \sum_{a=1}^{2}\left(p_{(a)} \cdot k\right)^{-1} \varepsilon_{\mu \rho} k_{\nu} k_{\sigma} \mathbf{j}_{(a)}^{\mu \nu} \mathbf{j}_{(a)}^{\rho \sigma}-\sum_{a=1}^{2} \frac{1}{M_{0} k^{0}} \varepsilon_{i \rho} k_{j} k_{\sigma} \mathbf{J}^{i j} \mathbf{j}_{(a)}^{\rho \sigma} \\
& -\frac{1}{2} \sum_{a=1}^{2} \frac{p_{(a)} \cdot k}{M_{0}^{2}\left(k^{0}\right)^{2}} \varepsilon_{i \ell} k_{j} k_{m} \mathbf{J}^{i j} \mathbf{J}^{\ell m} .
\end{aligned}
$$

Now we have $\mathbf{J} / M_{0} \sim a$, where $a$ is the size of the scatterer, so the last term is suppressed compared to the leading contribution $S_{\mathrm{gr}}^{(0)}$ - which is of order $m / \omega-$ by a factor of $(\omega a)^{2}$. We expect the non-universal terms, which are sensitive to the internal structure of the scatterer, to give contribution of similar order. Therefore we can trust (6.6) only in the regime in which $\left|\mathbf{j}_{(a)}^{\rho \nu}\right| \gg\left|\mathbf{J}^{i j}\right|$ so that the first two terms on the right hand side of (6.6) are large compared to the last term. This may be achieved e.g. by taking the impact parameter $b$ to be large compared to $a$, since in that case $\mathbf{j}_{(a)} / m \sim b$ and the contribution from the first two terms are of order $(m / \omega)(\omega b)^{2}$ and $(m / \omega)\left(\omega^{2} a b\right)$ respectively. In this case the soft expansion will be an expansion in powers of $\omega b$, and hold when $\omega b$ is small. We can now drop the last term in $S_{\mathrm{gr}}^{(2)}$ and express this as

$$
S_{\mathrm{gr}}^{(2)}=-\frac{1}{2} \sum_{a=1}^{2}\left(p_{(a)} \cdot k\right)^{-1} \varepsilon_{\mu \rho} k_{\nu} k_{\sigma} \mathbf{j}_{(a)}^{\mu \nu} \mathbf{j}_{(a)}^{\rho \sigma}-\sum_{a=1}^{2} \frac{1}{M_{0} k^{0}} \varepsilon_{i \rho} k_{j} k_{\sigma} \mathbf{J}^{i j} \mathbf{j}_{(a)}^{\rho \sigma} .
$$

We have to replace $S_{\mathrm{gr}}$ in (3.11) by $S_{\mathrm{gr}}^{(0)}+S_{\mathrm{gr}}^{(1)}+S_{\mathrm{gr}}^{(2)}$ to compute the angular power spectrum of soft gravitons to subsubleading order.

\subsection{Hidden assumptions}

The analysis for subleading soft photon and subsubleading soft graviton theorem is based on the assumptions that in the classical limit the soft factor for multiple soft particles is given by the product of single soft factors. This is the analog of (3.9). These results have not been established. However given the result for subleading soft gravitons, the fact that the consecutive soft limit always generates the product formula, and that in the classical limit the product formula is symmetric under the exchange of any pair of soft particles, we expect this result to be true. Extra contact terms would contain the contribution of the soft radiation to the soft factor, and we expect this contribution to be subdominant as long as the energy and angular momenta of the radiation remain small compared to those carried by the macroscopic objects. Nevertheless it will be useful to check this by explicit computation of the 'contact terms' similar to the one given in [78] and verifying that in the classical limit the contact term contribution is small compared to the product of single soft factors.

\section{Tests of soft theorem from classical radiation analysis}

In this section we shall verify / test the classical limits of soft theorems described above by explicitly analyzing classical electromagnetic / gravitational radiation during various scattering processes. 


\subsection{Soft photon theorem}

Let us consider a particle of charge $q$ moving along a trajectory $r(\sigma)=\left(r^{0}(\sigma), \vec{r}(\sigma)\right)$ labelled by the proper time $\sigma$ along the trajectory. Maxwell's equation takes the form

$$
\partial^{\mu} F_{\mu \nu}(x)=-j_{\nu}(x)=-q \int d \sigma \delta^{(D)}(x-r(\sigma)) V_{\nu}(\sigma),
$$

where

$$
V^{\alpha}(\sigma)=\frac{d r^{\alpha}}{d \sigma}
$$

This equation can be solved by setting

$$
A_{\alpha}(x)=-\int d \sigma G_{r}(x, r(\sigma)) q V_{\alpha}(\sigma),
$$

where $G_{r}\left(x, x^{\prime}\right)$ is the retarded Green's function, given by

$$
G_{r}\left(x, x^{\prime}\right)=\int \frac{d^{D} \ell}{(2 \pi)^{D}} e^{i \ell \cdot\left(x-x^{\prime}\right)} \frac{1}{\left(\ell^{0}+i \epsilon\right)^{2}-\vec{\ell}^{2}}
$$

satisfying

$$
\eta^{\mu \nu} \partial_{\mu} \partial_{\nu} G_{r}\left(x, x^{\prime}\right)=\delta^{(D)}\left(x-x^{\prime}\right), \quad G_{r}\left(x, x^{\prime}\right)=0 \quad \text { for } x^{0}<x^{\prime 0}
$$

This allows us to express the time Fourier transform of $A_{\alpha}(x)$ as

$$
\begin{aligned}
\widetilde{A}_{\alpha}(\omega, \vec{x}) & \equiv \int d x^{0} e^{i \omega x^{0}} A_{\alpha}\left(x^{0}, \vec{x}\right) \\
& =-\int d \sigma \int \frac{d^{D-1} \vec{\ell}}{(2 \pi)^{D-1}} e^{i \omega r^{0}(\sigma)+i \vec{\ell} \cdot\{\vec{x}-\vec{r}(\sigma)\}} \frac{1}{(\omega+i \epsilon)^{2}-\overrightarrow{\ell^{2}}} q V_{\alpha}(\sigma)
\end{aligned}
$$

We shall evaluate this for positive $\omega$ and later recover the result for negative $\omega$ using the reality of $A_{\alpha}(x)$. For fixed $\vec{x}$ and $\sigma$ we can decompose $\vec{\ell}$ into its component $\ell_{\|}$parallel to $\vec{x}-\vec{r}(\sigma)$ and the component $\vec{\ell}_{\perp}$ transverse to $\vec{x}-\vec{r}(\sigma)$. This gives

$$
\widetilde{A}_{\alpha}(\omega, \vec{x})=-\int d \sigma \int \frac{d^{D-2} \vec{\ell}_{\perp}}{(2 \pi)^{D-2}} \frac{d \ell_{\|}}{2 \pi} e^{i \omega r^{0}(\sigma)+i \ell_{\|}|\vec{x}-\vec{r}(\sigma)|} \frac{1}{(\omega+i \epsilon)^{2}-\ell_{\|}^{2}-\vec{\ell}_{\perp}^{2}} q V_{\alpha}(\sigma) .
$$

We can perform the integration over $\ell_{\|}$by closing its contour at infinity in the upper half plane, picking up residues at $\ell_{\|}=\sqrt{(\omega+i \epsilon)^{2}-\vec{\ell}_{\perp}^{2}}$. This gives

$$
\widetilde{A}_{\alpha}(\omega, \vec{x})=i \int d \sigma \int \frac{d^{D-2} \vec{\ell}_{\perp}}{(2 \pi)^{D-2}} e^{i \omega r^{0}(\sigma)+i \sqrt{(\omega+i \epsilon)^{2}-\vec{\ell}_{\perp}^{2}}|\vec{x}-\vec{r}(\sigma)|} \frac{1}{2 \sqrt{(\omega+i \epsilon)^{2}-\vec{\ell}_{\perp}^{2}}} q V_{\alpha}(\sigma)
$$

Our goal will be to evaluate this integral in the $|\vec{x}| \rightarrow \infty$ limit since we shall be interested in computing the radiative part of $\widetilde{A}_{\alpha}$. In this limit the integration over $\vec{\ell}_{\perp}$ is dominated by the stationary point of the exponent which is at $\vec{\ell}_{\perp}=0$. Since the dominant contribution 
to the integral is expected to come from the region close to $\vec{\ell}_{\perp}=0$, we can expand the exponent in power series in $\vec{\ell}_{\perp}$ :

$$
\widetilde{A}_{\alpha}(\omega, \vec{x}) \simeq i \int d \sigma \int \frac{d^{D-2} \vec{\ell}_{\perp}}{(2 \pi)^{D-2}} e^{i \omega r^{0}(\sigma)+i\left\{\omega+i \epsilon-\frac{\vec{\ell}_{\perp}^{2}}{2(\omega+i \epsilon)}\right\}|\vec{x}-\vec{r}(\sigma)|+\cdots}\left\{\frac{1}{2(\omega+i \epsilon)}+\cdots\right\} q V_{\alpha}(\sigma),
$$

where the $\cdots$ denote terms containing higher powers of $\vec{\ell}_{\perp}^{2}$, which, after $\vec{\ell}_{\perp}$ integration, will turn into higher powers of $1 /|\vec{x}|$. The $i \epsilon$ term provides the required damping term $\exp \left[-\epsilon|\vec{x}-\vec{r}(\sigma)| \vec{\ell}_{\perp}^{2} /\left(2 \omega^{2}\right)\right]$ for the $\vec{\ell}_{\perp}$ integral. We can carry out the $\vec{\ell}_{\perp}$ integration using the standard formula for gaussian integration. This gives

$$
\begin{aligned}
\widetilde{A}_{\alpha}(\omega, \vec{x}) & \simeq i\left(\frac{\omega}{2 \pi i|\vec{x}|}\right)^{(D-2) / 2} \frac{1}{2 \omega} \int d \sigma e^{i \omega r^{0}(\sigma)+i \omega|\vec{x}-\vec{r}(\sigma)|} q V_{\alpha}(\sigma) \\
& \simeq i \mathcal{N} e^{i \omega R} \int d \sigma e^{i \omega\left\{r^{0}(\sigma)-\hat{n} . \vec{r}(\sigma)\right\}} q V_{\alpha}(\sigma),
\end{aligned}
$$

where $\hat{n}$ denotes unit vector along $\vec{x}$, and

$$
R \equiv|\vec{x}|, \quad \mathcal{N}=\left(\frac{\omega}{2 \pi i R}\right)^{(D-2) / 2} \frac{1}{2 \omega} .
$$

Even though (7.10) was derived for positive $\omega$, using the reality condition $\left(\widetilde{A}_{\alpha}(\omega, \vec{x})\right)^{*}=$ $\widetilde{A}_{\alpha}(-\omega, \vec{x})$, we now see that $(7.10)$ holds for negative $\omega$ as well.

We now note that for a given particle trajectory, as $\sigma \rightarrow \pm \infty, q V_{\alpha}(\sigma)$ approaches a constant, and $\left\{r^{0}(\sigma)-\hat{n} . \vec{r}(\sigma)\right\}$ approaches $\left(V^{0}-\hat{n} . \vec{V}\right) \sigma$ plus a constant. Therefore the integrand is oscillatory. This is defined using the standard trick that sets $\int_{a}^{\infty} d s e^{i \omega s}$ to $-e^{i \omega a} /(i \omega)$, ignoring the boundary terms at infinity. This is equivalent to explicitly adding terms that cancel the boundary terms. Using this insight we make (7.10) well defined by adding to it appropriate boundary terms:

$$
\begin{aligned}
\widetilde{A}_{\alpha}(\omega, \vec{x}) \simeq & i \mathcal{N} e^{i \omega R} \int_{\sigma_{-}}^{\sigma_{+}} d \sigma e^{i \omega\left\{r^{0}(\sigma)-\hat{n} . \vec{r}(\sigma)\right\}} q V_{\alpha}(\sigma) \\
& -\mathcal{N} e^{i \omega R} \frac{1}{\omega}\left[e^{i \omega\left\{r^{0}(\sigma)-\hat{n} . \vec{r}(\sigma)\right\}}\left\{\frac{q V_{\alpha}(\sigma)}{V^{0}(\sigma)-\hat{n} \cdot \vec{V}(\sigma)}\right\}\right]_{\sigma_{-}}^{\sigma_{+}},
\end{aligned}
$$

where $\sigma_{ \pm}$are the limits of integration with the understanding that we have to take the limit $\sigma_{ \pm} \rightarrow \pm \infty$ at the end.

Let us define

$$
V_{ \pm}=V\left(\sigma_{ \pm}\right), \quad r_{ \pm}=r\left(\sigma_{ \pm}\right), \quad k=-\omega(1, \hat{n}),
$$

where the minus sign in the expression for $k$ reflects that $k$ represents the momentum of an outgoing photon. We now expand all the terms on the right hand side of (7.12), except the $e^{i \omega R}$ factor, in a power series in $\omega$. This gives, for any polarization vector $\varepsilon$,

$$
\varepsilon^{\alpha} \widetilde{A}_{\alpha}=\mathcal{N} e^{i \omega R}\left[i q\left(\varepsilon \cdot r_{+}-\varepsilon \cdot r_{-}\right)-\frac{q \varepsilon \cdot V_{+}}{k \cdot V_{+}}\left(1+i k \cdot r_{+}\right)+\frac{q \varepsilon \cdot V_{-}}{k \cdot V_{-}}\left(1+i k \cdot r_{-}\right)+\mathcal{O}(\omega)\right]
$$


Taking the trajectories in the far past and far future to be of the form

$$
r(\sigma)=c_{ \pm}+V_{ \pm} \sigma
$$

we get

$$
r_{ \pm}=c_{ \pm}+V_{ \pm} \sigma_{ \pm}
$$

Substituting this into (7.14) we get

$$
\begin{gathered}
\varepsilon \cdot \widetilde{A}=\mathcal{N} e^{i \omega R}\left[-q\left\{\frac{\varepsilon \cdot V_{+}}{k \cdot V_{+}}-\frac{\varepsilon \cdot V_{-}}{k \cdot V_{-}}\right\}-\frac{i q}{k \cdot V_{+}}\left\{\varepsilon \cdot V_{+} k \cdot c_{+}-\varepsilon \cdot c_{+} k \cdot V_{+}\right\}\right. \\
\left.+\frac{i q}{k \cdot V_{-}}\left\{\varepsilon \cdot V_{-} k \cdot c_{-}-\varepsilon \cdot c_{-} k \cdot V_{-}\right\}+\mathcal{O}(\omega)\right] .
\end{gathered}
$$

Now for this problem, we have

$$
p_{(1)}=m V_{-}, \quad p_{(2)}=-m V_{+}, \quad c_{(1)}=c_{-}, \quad c_{(2)}=c_{+},
$$

where $m$ is the mass of the particle and $c_{(1)}$ and $c_{(2)}$ are the constants introduced in (3.32). The minus sign in the expression for $p_{(2)}$ accounts for the fact that it is the momentum of an outgoing particle. Substituting these into (6.3) we get

$$
S_{\mathrm{em}}=\left[-q\left\{\frac{\varepsilon \cdot V_{+}}{k \cdot V_{+}}-\frac{\varepsilon \cdot V_{-}}{k \cdot V_{-}}\right\}-\frac{i q}{k \cdot V_{+}}\left\{\varepsilon \cdot V_{+} k \cdot c_{+}-\varepsilon \cdot c_{+} k \cdot V_{+}\right\}+\frac{i q}{k \cdot V_{-}}\left\{\varepsilon \cdot V_{-} k \cdot c_{-}-\varepsilon \cdot c_{-} k \cdot V_{-}\right\}\right] .
$$

Comparing this with (7.17) we get, up to subleading order in $\omega$,

$$
\varepsilon \cdot \widetilde{A}=\mathcal{N} e^{i \omega R} S_{\mathrm{em}}(\varepsilon, k) .
$$

We shall now use this to compute the energy carried away by photons of frequency between $\omega$ and $\omega(1+\delta)$ within a solid angle $\Delta \Omega$ around $\hat{n}$. To do this we note that for normalized $\varepsilon, \varepsilon . A$ can be regarded as a real scalar field $\phi$. Therefore $\epsilon . \widetilde{A}$ gives its time Fourier transform $\tilde{\phi}(\omega, \vec{x})$. Furthermore far away from the source the radiation looks like a plane wave propagating along the direction $\hat{n}$ with no dependence on the transverse coordinates. If we denote the radial coordinate along $\hat{n}$ around the relevant region by $z \equiv \hat{n} . \vec{x}=|\vec{x}|=R$, then the relevant part of $\widetilde{\phi}(\omega, \vec{x})$ has the form

$$
\widetilde{\phi}(\omega, \vec{x})=e^{i \omega z} \mathcal{N} S_{\mathrm{em}}(\varepsilon, k) .
$$

Now the energy momentum tensor of $\phi$ is given by

$$
T_{\mu \nu}=\partial_{\mu} \phi \partial_{\nu} \phi-\frac{1}{2} \eta_{\mu \nu} \partial^{\rho} \phi \partial_{\rho} \phi .
$$

Therefore the total energy flow per unit 'area' along $z$ direction is

$$
\int d z T^{z t}=\int d z \int \frac{d \omega}{2 \pi} \int \frac{d \omega^{\prime}}{2 \pi}\left(-\omega \omega^{\prime}\right) e^{-i\left(\omega+\omega^{\prime}\right) t} \tilde{\phi}(\omega, z) \tilde{\phi}\left(\omega^{\prime}, z\right) .
$$


Using (7.21) we see that the $z$ integral produces a factor of $2 \pi \delta\left(\omega+\omega^{\prime}\right)$. Using the fact that $\tilde{\phi}(-\omega, z)=\tilde{\phi}(\omega, z)^{*}$, we can express $(7.23)$ as

$$
\int_{-\infty}^{\infty} \frac{d \omega}{2 \pi} \omega^{2}|\mathcal{N}|^{2}\left|S_{\mathrm{em}}(\varepsilon, k)\right|^{2}=\int_{0}^{\infty} \frac{d \omega}{\pi} \omega^{2}|\mathcal{N}|^{2}\left|S_{\mathrm{em}}(\varepsilon, k)\right|^{2} .
$$

A solid angle $\Delta \Omega$ corresponds to an 'area' $R^{D-2} \Delta \Omega$. Therefore the total energy carried by the photons of polarization $\varepsilon$, within solid angle $\Delta \Omega$ of $\hat{n}$, with frequency between $\omega$ and $\omega(1+\delta)$ is given by

$$
R^{D-2} \Delta \Omega \omega \delta \frac{1}{\pi} \omega^{2}|\mathcal{N}|^{2}\left|S_{\mathrm{em}}(\varepsilon, k)\right|^{2} .
$$

Substituting the value of $\mathcal{N}$ from (7.11) we can reduce (7.25) to

$$
\frac{1}{2}(2 \pi)^{-(D-1)} \Delta \Omega \omega^{D-1} \delta\left|S_{\mathrm{em}}(\varepsilon, k)\right|^{2} .
$$

This is in perfect agreement with $(2.7)$ with $S_{\mathrm{em}}^{(0)}$ replaced by $S_{\mathrm{em}}=S_{\mathrm{em}}^{(0)}+S_{\mathrm{em}}^{(1)}$. An important point to note is that the normalization constant $\mathcal{N}$ contains information about the $\omega$-dependent phase space factor that was used to get (2.7).

One could also try to give an alternate derivation of (7.12) by using the position space representation of the retarded Green's function $G_{r}\left(x, x^{\prime}\right)$. In even space-time dimensions the retarded Green's function is proportional to $\theta\left(x^{0}-x^{\prime 0}\right) \delta\left(\left(x-x^{\prime}\right)^{2}\right)$ and one could use the analysis given in section 14 of [79] to derive an expression for the gauge field produced by a moving charged particle. In odd space-time dimensions the retarded Green's function has a more complicated form, but presumably the radiative component of the field can be found without too much hurdle.

\subsection{Soft graviton theorem}

Eq. (7.17) for electromagnetic radiation was derived for an arbitrary trajectory of a charged particle, moving under some unspecified force. This is possible in electromagnetism since the conservation of electromagnetic current of a point source does not require the source to satisfy its equation of motion. However for gravity this is not so - we need the source to satisfy its equation of motion in order that it generates a conserved energy-momentum tensor. This means that we have to take into account all forces acting on the source during its motion and take into account the gravitational radiation generated by the fields responsible for these forces in order to arrive at a consistent result. We shall now describe some situations where the computation can be done and compare the corresponding results with the prediction of soft theorem. Similar calculations of gravitational radiation from classical point sources have been performed in different context recently in [98-100].

\subsubsection{Scattering via fusion and decay:}

In [85] Weinberg considered the situation where a bunch of particles moving along straight lines meet at a single point in space-time and come out as another bunch of particles emerging from the same space-time point and moving along straight line trajectories. Physically this corresponds to the situation where there is a repulsive short-range interaction between 


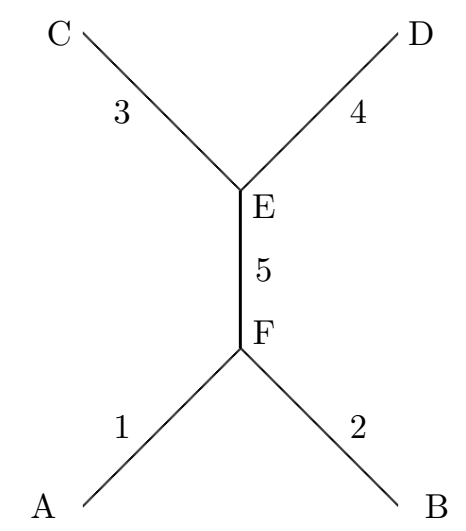

Figure 1. A scattering process in space-time.

the particles where the range of the interaction is large compared to the Schwarzschild radii of the particles. For this reason the effect of gravitational interaction between the particles can be ignored and the particles can be taken to move along straight lines till the repulsive interaction switches on. If we now consider long wave-length gravitational waves - with wave-length much larger than the range of the repulsive interaction - then the interaction can regarded as contact interaction. This leads to the picture described above. Weinberg showed that this analysis produces correctly the leading soft graviton theorem. In fact for this analysis the probe limit was not necessary - one could reproduce the full result (3.2).

One could now ask if we can work to higher order and verify the subleading (or even subsubleading) contribution to the soft graviton theorem. The problem with this is that since the external particle trajectories meet at a single point in space-time, all the orbital angular momenta vanish if we choose the common meeting point as the origin of space-time and there is no subleading contribution left over. We shall now consider a slightly different type of scattering that overcomes this problem. We shall assume that the interaction is such that once the initial particles collide at a single space-time point, they fuse and travel as a single entity for certain distance, and then splits apart into final state particles which travel away in different directions along straight line trajectories. This has been shown in figure 1 for $2 \rightarrow 2$ scattering. Since the energy momentum tensor is conserved locally during this process, this is a consistent source. However in this case the orbital angular momentum of the particles no longer vanish identically and we have a non-zero contribution from the subleading term in the soft theorem. This can then be compared with the result of direct computation.

During this analysis we shall not need to take the probe limit. This is a reflection of the fact that the regime we are considering, in which the interaction length scale is much larger then the Schwarzschild radius of the black hole, the upper cut-off on the frequency of gravitational wave remains small compared to the inverse Schwarzschild radius of the black hole. As a result the total energy carried away by the radiation remains small compared to the incoming and outgoing particle masses even without taking the probe limit. We also expect the analysis to reproduce the results of soft theorem to subsubleading order, since in this case the subsubleading contribution comes only from the orbital angular momentum 
part. The possible non-universal terms would come from non-minimal effective coupling of gravity to the external states, and we shall assume that the external states have only minimal coupling to gravity.

To compute the gravitational radiation from this process, we use the standard formula for the gravitational field $h_{\alpha \beta}(x)$ of a point source of mass $m$, obtained from the convolution of the retarded Green's function $G_{r}(x, y)$ defined in (7.4) with $-T_{\alpha \beta}(y)+(D-2)^{-1} \eta_{\alpha \beta} T_{\gamma}^{\gamma}(y)$ where $T_{\alpha \beta}$ denotes the energy momentum tensor of the source. This is a straightforward generalization of the corresponding formula in four space-time dimensions as given in [85]. We have

$$
\begin{aligned}
& h_{\alpha \beta}(x)=e_{\alpha \beta}(x)-\frac{1}{D-2} \eta_{\alpha \beta} e_{\gamma}^{\gamma}(x), \\
& e_{\alpha \beta}(x) \equiv-\int d^{D} x^{\prime} G_{r}\left(x, x^{\prime}\right) T_{\alpha \beta}\left(x^{\prime}\right)=-\int d \sigma G_{r}(x, r(\sigma)) P_{\alpha}(\sigma) V_{\beta}(\sigma),
\end{aligned}
$$

where $r(\sigma)$ describes the trajectory of the particle parametrized by the proper time $\sigma$, $V=d r / d \sigma$ is the four velocity of the particle and $P=m V$ is the four momentum of the particle. Following the same steps that led from (7.3) to (7.12), we can obtain the long distance behavior of (7.28) to be

$$
\begin{aligned}
\tilde{e}_{\alpha \beta}= & i \mathcal{N} e^{i \omega R} \int d \sigma e^{i \omega\left\{r^{0}(\sigma)-\hat{n} \cdot \vec{r}(\sigma)\right\}} P_{\alpha}(\sigma) V_{\beta}(\sigma) \\
& -\mathcal{N} e^{i \omega R} \frac{1}{\omega}\left[e^{i \omega\left\{r^{0}(\sigma)-\hat{n} . \vec{r}(\sigma)\right\}}\left\{\frac{P_{\alpha}(\sigma) V_{\beta}(\sigma)}{V^{0}(\sigma)-\hat{n} \cdot \vec{V}(\sigma)}\right\}\right]_{\text {boundary }},
\end{aligned}
$$

where $\mathcal{N}$ is the same normalization constant as defined in (7.11). The integration over $\sigma$ in the first line runs over all the trajectory segments, and the boundary terms given in the second line receives contribution from the outer ends of all the external lines. Explicitly evaluating the boundary contributions to (7.29) from the four end points $A, B, C$ and $D$, we can express (7.29) as

$$
\begin{aligned}
\tilde{e}_{\alpha \beta} \simeq-\mathcal{N} e^{i \omega R}[ & -\sum_{a=1}^{2} e^{i k \cdot r_{(a)}} \frac{P_{(a) \alpha} P_{(a) \beta}}{k \cdot P_{(a)}} \\
& \left.+\sum_{a=3}^{4} e^{i k \cdot r_{(a)}} \frac{P_{(a) \alpha} P_{(a) \beta}}{k \cdot P_{(a)}}-i \sum_{a=1}^{5} \int d \sigma_{a} e^{i k \cdot r(\sigma)} P_{(a) \alpha} V_{(a) \beta}\right]
\end{aligned}
$$

where $\sigma_{a}$ is the proper time parametrizing the $a$-th trajectory segment, $P_{(a)}$ is the momentum flowing along the $a$-th segment along the direction of increasing $\sigma_{a}, r_{(a)}$ for $a=1,2,3,4$ are the locations of the four space-time points $A, B, C$ and $D$ in figure 1 and $k=-\omega(1, \hat{n})$. Since the external momenta are considered to be positive if they are ingoing, we have

$$
\begin{array}{ll}
P_{(a)}=p_{(a)} & \text { for } a=1,2, \\
P_{(a)}=-p_{(a)} & \text { for } a=3,4, \quad P_{(5)}=p_{(1)}+p_{(2)}=-p_{(3)}-p_{(4)}, \\
V_{(a)}=v_{(a)} & \text { for } a=1,2, \\
V_{(a)}=-v_{(a)} & \text { for } a=3,4
\end{array}
$$


where $v_{(a)}=p_{(a)} / m_{(a)}, m_{(a)}$ being the mass of the $a$-th external state. Now let us choose the origin of space-time coordinates to be at $F$, and let us choose the convention that $\sigma_{1}$, $\sigma_{2}$ and $\sigma_{5}$ vanish at $F$ and $\sigma_{3}$ and $\sigma_{4}$ vanish at $E$. Then we have

$$
r\left(\sigma_{a}\right)=V_{(a)} \sigma_{a} \quad \text { for } a=1,2,5, \quad r\left(\sigma_{a}\right)=r(E)+V_{(a)} \sigma_{a} \quad \text { for } a=3,4
$$

Let us suppose further that we have

$$
\sigma_{1}=-s_{1} \text { at } A, \quad \sigma_{2}=-s_{2} \text { at } B, \quad \sigma_{3}=s_{3} \text { at } C, \quad \sigma_{4}=s_{4} \text { at } D, \quad \sigma_{5}=s_{5} \text { at } E .
$$

This gives, by integrating the $d r / d \tau=V$ equation:

$$
\begin{aligned}
r_{(1)} & =-s_{1} v_{(1)}, \quad r_{(2)}=-s_{2} v_{(2)}, \quad r_{(3)}=-s_{3} v_{(3)}+r(E), \quad r_{(4)}=-s_{4} v_{(4)}+r(E), \\
r(E) & =V_{(5)} s_{5} .
\end{aligned}
$$

Finally, since $v_{(a)}$ is parallel to $p_{(a)}$, we have

$$
\frac{k \cdot v_{(a)}}{k \cdot p_{(a)}} p_{(a) \alpha} p_{(a) \beta}=p_{(a) \alpha} v_{(a) \beta} .
$$

Using these relations we can express (7.30) up to subsubleading order in $k$ as:

$$
\begin{aligned}
\tilde{e}_{\alpha \beta}=\mathcal{N} e^{i \omega R}[ & \sum_{a=1}^{4} \frac{p_{(a) \alpha} p_{(a) \beta}}{k \cdot p_{(a)}}+i \sum_{a=3}^{4} \frac{1}{k \cdot p_{(a)}} p_{(a) \alpha} k^{\gamma}\left\{p_{(a) \beta} r_{\gamma}(E)-p_{(a) \gamma} r_{\beta}(E)\right\} \\
& \left.-\frac{1}{2} \sum_{a=3,4}(k \cdot r(E))^{2} \frac{p_{(a) \alpha} p_{(a) \beta}}{k \cdot p_{(a)}}+\frac{1}{2} \sum_{a=3,4} p_{(a) \alpha} r(E)_{\beta} k \cdot r(E)\right] .
\end{aligned}
$$

We can now compute $\tilde{h}_{\alpha \beta}$ using (7.27):

$$
\tilde{h}_{\alpha \beta}=\tilde{e}_{\alpha \beta}-\frac{1}{D-2} \eta_{\alpha \beta} \tilde{e}_{\gamma}^{\gamma}
$$

However our interest will be on the projection of $\tilde{h}$ along a given polarization $\varepsilon$. This is obtained by contracting $\varepsilon$ with $\tilde{h}$ via the metric given on the right hand side of (4.8). This gives the projection to be

$$
\phi(\varepsilon) \equiv \varepsilon^{\alpha \beta} \tilde{h}_{\alpha \beta}-\frac{1}{2} \varepsilon_{\alpha}^{\alpha} \tilde{h}_{\beta}^{\beta}=\varepsilon^{\alpha \beta} \tilde{e}_{\alpha \beta} .
$$

Using (7.37) we now get

$$
\begin{aligned}
\phi(\varepsilon)= & \mathcal{N} e^{i \omega R}\left[\sum_{a=1}^{4} \frac{\varepsilon^{\alpha \beta} p_{(a) \alpha} p_{(a) \beta}}{k \cdot p_{(a)}}+i \sum_{a=3}^{4} \frac{1}{k \cdot p_{(a)}} \varepsilon^{\alpha \beta} p_{(a) \alpha} k^{\gamma}\left\{p_{(a) \beta} r_{\gamma}(E)-p_{(a) \gamma} r_{\beta}(E)\right\}\right. \\
& \left.-\frac{1}{2} \sum_{a=3,4}(k \cdot r(E))^{2} \frac{\varepsilon^{\alpha \beta} p_{(a) \alpha} p_{(a) \beta}}{k \cdot p_{(a)}}+\frac{1}{2} \sum_{a=3,4} \varepsilon^{\alpha \beta} p_{(a) \alpha} r(E)_{\beta} k \cdot r(E)\right]
\end{aligned}
$$




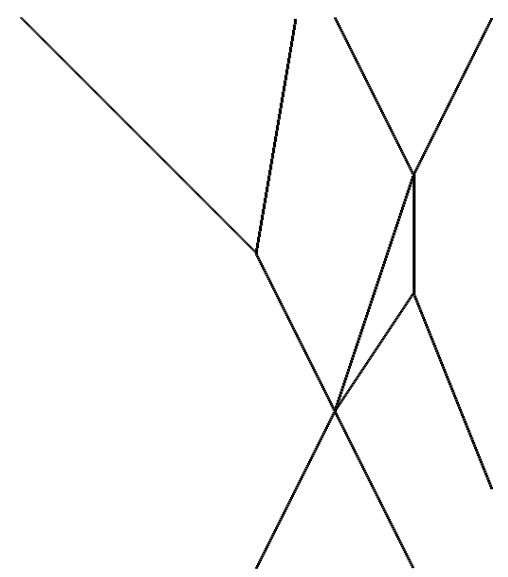

Figure 2. A more complicated scattering process in space-time.

On the other hand here the orbital angular momenta of the external particles, measured with respect to the origin of space-time situated at $F$, are given by

$$
\mathbf{J}_{(a) \alpha \beta}=0 \quad \text { for } a=1,2, \quad \mathbf{J}_{(a) \alpha \beta}=r_{\alpha}(E) p_{(a) \beta}-r_{\beta}(E) p_{(a) \alpha} \quad \text { for } a=3,4 .
$$

Using this, and the fact that $r(E)$ is proportional to $p_{(3)}+p_{(4)}$ so that $k . r(E)\left(p_{(3) \alpha}+p_{(4) \alpha}\right)=$ $k .\left(p_{(3)}+p_{(4)}\right) r(E)_{\alpha}$, we can express (7.40) as

$$
\begin{aligned}
\phi(\varepsilon)=\mathcal{N} e^{i \omega R}[ & \sum_{a=1}^{4} \frac{\varepsilon^{\alpha \beta} p_{(a) \alpha} p_{(a) \beta}}{k \cdot p_{(a)}} \\
& \left.+i \sum_{a=1}^{4} \frac{1}{k \cdot p_{(a)}} \varepsilon^{\alpha \beta} p_{(a) \alpha} k^{\gamma} \mathbf{J}_{(a) \gamma \beta}-\frac{1}{2} \sum_{a=1}^{4} \frac{1}{k \cdot p_{(a)}} \varepsilon_{\alpha \beta} k_{\gamma} k_{\delta} \mathbf{J}_{(a)}^{\alpha \gamma} \mathbf{J}_{(a)}^{\beta \delta}\right] \\
=\mathcal{N} e^{i \omega R}[ & \left.S_{\mathrm{gr}}^{(0)}(\varepsilon, k)+S_{\mathrm{gr}}^{(1)}(\varepsilon, k)+S_{\mathrm{gr}}^{(2)}(\varepsilon, k)\right] .
\end{aligned}
$$

We can now use the same analysis that led from (7.20) to (7.26) to show that the angular power spectrum of soft graviton radiation during this process is given by eq. (3.10).

\subsubsection{Scattering via multiple fusion and decay}

We shall now consider a more complicated scattering via multiple interactions of the type shown in figure 2. The rules are the same as in section 7.2.1, i.e. the energy momentum tensor is localized along the trajectories and is conserved at each vertex. We shall denote by $p_{(a)}$ for $a=1, \cdots n$ the external momenta, and all of them will be taken to be ingoing. We shall also denote by $P_{(s)}$ and $V_{(s)}$ the momenta and velocities along all the trajectory segments - both external and internal, along the direction of increasing proper time. Then the analog of (7.30) takes the form

$$
\tilde{e}_{\alpha \beta} \simeq-\mathcal{N} e^{i \omega R}\left[-\sum_{a=1}^{n} e^{i k \cdot r_{(a)}} \frac{p_{(a) \alpha} p_{(a) \beta}}{k \cdot p_{(a)}}-i \sum_{s} \int d \sigma_{s} e^{i k \cdot r\left(\sigma_{s}\right)} P_{(s) \alpha} V_{(s) \beta}\right]
$$


where $r_{(a)}$ denotes the space-time coordinates of the boundary of the $a$-th external trajectory and $\sigma_{s}$ is the proper time along the $s$-th trajectory segment. We now expand the integrand in power series in $k$ up to subsubleading order and carry out integration over $\sigma_{s}$ using the fact that along the $s$-th trajectory

$$
r\left(\sigma_{s}\right)=I_{s}+\sigma_{s} V_{(s)},
$$

where we define

$$
I_{s} \equiv r\left(\sigma_{s, i}\right), \quad J_{s} \equiv r\left(\sigma_{s, f}\right),
$$

$\sigma_{s, i}$ and $\sigma_{s, f}$ being the initial and final values of $\sigma_{s}$ along the $s$-th trajectory. With this we can express (7.43) as

$$
\begin{aligned}
\tilde{e}_{\alpha \beta} \simeq & \mathcal{N} e^{i \omega R}\left[\sum_{a=1}^{n}\left\{1+i k \cdot r_{(a)}-\frac{1}{2}\left(k \cdot r_{(a)}\right)^{2}\right\} \frac{p_{(a) \alpha} p_{(a) \beta}}{k \cdot p_{(a)}}\right. \\
& \left.+i \sum_{s} P_{(s) \alpha} V_{(s) \beta}\left\{\left(\sigma_{s, f}-\sigma_{s, i}\right)+i k \cdot I_{s}\left(\sigma_{s, f}-\sigma_{s, i}\right)+\frac{i}{2} k \cdot V_{(s)}\left(\sigma_{s, f}-\sigma_{s, i}\right)^{2}\right\}\right] .
\end{aligned}
$$

Now it follows from $(7.44),(7.45)$ that

$$
J_{s}-I_{s}=V_{(s)}\left(\sigma_{s, f}-\sigma_{s, i}\right) .
$$

Using this we can express (7.46) as

$$
\begin{aligned}
\tilde{e}_{\alpha \beta} \simeq \mathcal{N} e^{i \omega R}[ & \sum_{a=1}^{n}\left\{1+i k \cdot r_{(a)}-\frac{1}{2}\left(k \cdot r_{(a)}\right)^{2}\right\} \frac{p_{(a) \alpha} p_{(a) \beta}}{k \cdot p_{(a)}} \\
& \left.+i \sum_{s} P_{(s) \alpha}\left\{\left(J_{s}-I_{s}\right)_{\beta}+i k . I_{s}\left(J_{s}-I_{s}\right)_{\beta}+\frac{i}{2} k .\left(J_{s}-I_{s}\right)\left(J_{s}-I_{s}\right)_{\beta}\right\}\right] .
\end{aligned}
$$

Consider now the sum $\sum_{s} P_{(s) \alpha}\left(J_{s}-I_{s}\right)_{\beta}$. We can rearrange this by fixing a given vertex and summing over all the lines connected to the vertex. This will give a sum over all the momenta entering a vertex which will vanish by momentum conservation. The only exceptions are the outer ends of the external trajectories. This contribution gives

$$
\sum_{s} P_{(s) \alpha}\left(J_{s}-I_{s}\right)_{\beta}=-\sum_{a=1}^{n} r_{(a) \beta} p_{(a) \alpha},
$$

where the minus sign reflects the fact that the momenta $p_{(a)}$ are directed away from these ends.

Next consider the sum

$$
\begin{aligned}
& \sum_{s} P_{(s) \alpha}\left\{k \cdot I_{s}\left(J_{s}-I_{s}\right)_{\beta}+\frac{1}{2} k .\left(J_{s}-I_{s}\right)\left(J_{s}-I_{s}\right)_{\beta}\right\} \\
= & \frac{1}{2} \sum_{s} P_{(s) \alpha}\left\{k . J_{s}\left(J_{s}\right)_{\beta}-k . I_{s}\left(I_{s}\right)_{\beta}-k . J_{s}\left(I_{s}\right)_{\beta}+k . I_{s}\left(J_{s}\right)_{\beta}\right\} .
\end{aligned}
$$


Substituting (7.49) and (7.50) into (7.48) we get

$$
\varepsilon^{\alpha \beta} \tilde{e}_{\alpha \beta} \simeq \mathcal{N} e^{i \omega R} \bar{S}_{\mathrm{gr}},
$$

where

$$
\begin{aligned}
\bar{S}_{\mathrm{gr}}= & \varepsilon^{\alpha \beta}\left[\sum_{a=1}^{n}\left\{1+i k \cdot r_{(a)}\right\} \frac{p_{(a) \alpha} p_{(a) \beta}}{k \cdot p_{(a)}}-i \sum_{a=1}^{n} r_{(a) \beta} p_{(a) \alpha}-\frac{1}{2} \sum_{a=1}^{n}\left(k \cdot r_{(a)}\right)^{2} \frac{p_{(a) \alpha} p_{(a) \beta}}{k \cdot p_{(a)}}\right. \\
& \left.\quad-\frac{1}{2} \sum_{s} P_{(s) \alpha}\left\{k \cdot J_{s}\left(J_{s}\right)_{\beta}-k \cdot I_{s}\left(I_{s}\right)_{\beta}-k \cdot J_{s}\left(I_{s}\right)_{\beta}+k \cdot I_{s}\left(J_{s}\right)_{\beta}\right\}\right] \\
\equiv & \bar{S}_{\mathrm{gr}}^{(0)}+\bar{S}_{\mathrm{gr}}^{(1)}+\bar{S}_{\mathrm{gr}}^{(2)} .
\end{aligned}
$$

$\bar{S}_{\mathrm{gr}}^{(0)}, \bar{S}_{\mathrm{gr}}^{(1)}$ and $\bar{S}_{\mathrm{gr}}^{(2)}$ represent respectively the leading, subleading and subsubleading contributions to $(7.52)$.

Our goal will be to show that $\bar{S}_{\text {gr }}$ given in (7.52) agrees with the soft factor $S_{\mathrm{gr}}^{(0)}+$ $S_{\mathrm{gr}}^{(1)}+S_{\mathrm{gr}}^{(2)}$. Now for this problem we have

$$
\mathbf{J}_{(a)}^{\mu \nu}=r_{(a)}^{\mu} p_{(a)}^{\nu}-r_{(a)}^{\nu} p_{(a)}^{\mu} .
$$

Using this and (3.2), (3.6), (6.5) we get

$$
\begin{aligned}
S_{\mathrm{gr}} \equiv S_{\mathrm{gr}}^{(0)} & +S_{\mathrm{gr}}^{(1)}+S_{\mathrm{gr}}^{(2)} \\
=\varepsilon^{\alpha \beta} & {\left[\sum_{a=1}^{n} \frac{p_{(a) \alpha} p_{(a) \beta}}{k \cdot p_{(a)}}+i \sum_{a=1}^{n}\left\{\frac{p_{(a) \alpha} p_{(a) \beta}}{k \cdot p_{(a)}} k \cdot r_{(a)}-r_{(a) \beta} p_{(a) \alpha}\right\}\right.} \\
& -\frac{1}{2} \sum_{a=1}^{n} \frac{1}{k \cdot p_{(a)}}\left\{p_{(a) \alpha} p_{(a) \beta}\left(k \cdot r_{(a)}\right)^{2}+\left(k \cdot p_{(a)}\right)^{2} r_{(a) \alpha} r_{(a) \beta}\right. \\
& \left.\left.-k \cdot p_{(a)} k \cdot r_{(a)} p_{(a) \alpha} r_{(a) \beta}-k \cdot p_{(a)} k \cdot r_{(a)} r_{(a) \alpha} p_{(a) \beta}\right\}\right] .
\end{aligned}
$$

Comparing the first line of (7.52) and the second line of (7.54) we see that $\bar{S}_{\mathrm{gr}}$ and $S_{\mathrm{gr}}$ agree up to subleading order. We shall now show that this equality also holds at the subsubleading order.

One property of (7.52) that we shall use to simplify our analysis is that this expression is independent of the length of the external legs, i.e. it remains invariant under the transformation $r_{(a)} \rightarrow r_{(a)}+b_{(a)} p_{(a)}$ for arbitrary constants $b_{(a)}$. This can be verified explicitly, but also follows from the original expression (7.43) which by construction is independent of the outer limits of $\sigma$ integration for the external lines. Using this property we can even take the external lines to have zero length. (7.54) shares a similar property that follows from the fact that $\mathbf{J}_{(a)}^{\mu \nu}$ defined in (7.53) is invariant under $r_{(a)} \rightarrow r_{(a)}+b_{(a)} p_{(a)}$.

Our proof of the equality of $S_{\mathrm{gr}}^{(2)}$ and $\bar{S}_{g r}^{(2)}$ will proceed by induction. First we shall assume that it holds for all tree diagrams with $n$ external legs and prove that it also holds for all tree diagrams with $n+1$ external legs. Then we shall assume that it holds for all 
diagrams with at most $\ell$ loops and then prove that it also holds for all diagrams with at most $\ell+1$ loops.

Let us begin with the first step. Suppose we have an $n+1$ point amplitude in which the external lines carrying momentum $p_{(u)}$ and $p_{(v)}$ join at a space-time point $r$ to produce an internal line of momentum $p_{(u)}+p_{(v)}$. By taking the external legs carrying momenta $p_{(u)}$ and $p_{(v)}$ to have zero length, we take $r_{(u)}=r_{(v)}=r$. We compare this with another diagram with $n$ external legs where the pair of external lines carrying momenta $p_{(u)}$ and $p_{(v)}$ are removed and the internal line carrying momentum $p_{(u)}+p_{(v)}$ is regarded as an external line. From (7.54) we see that the difference in $S_{\mathrm{gr}}^{(2)}$ between the first and the second diagram is given by

$$
\begin{aligned}
\Delta S_{\mathrm{gr}}^{(2)}= & -\frac{1}{2}(k \cdot r)^{2} \varepsilon_{\alpha \beta}\left\{\frac{p_{(u)}^{\alpha} p_{(u)}^{\beta}}{k \cdot p_{(u)}}+\frac{p_{(v)}^{\alpha} p_{(v)}^{\beta}}{k \cdot p_{(v)}}\right\}-\frac{1}{2} \varepsilon^{\alpha \beta} r_{\alpha} r_{\beta} k \cdot\left\{p_{(u)}+p_{(v)}\right\} \\
& +\varepsilon^{\alpha \beta} r_{\alpha}\left\{p_{(u) \beta}+p_{(v) \beta}\right\} k \cdot r+\frac{1}{2}(k \cdot r)^{2} \varepsilon_{\alpha \beta} \frac{\left\{p_{(u)}^{\alpha}+p_{(v)}^{\alpha}\right\}\left\{p_{(u)}^{\beta}+p_{(v)}^{\beta}\right\}}{k \cdot\left\{p_{(u)}+p_{(v)}\right\}} \\
& +\frac{1}{2} \varepsilon^{\alpha \beta} r_{\alpha} r_{\beta} k \cdot\left\{p_{(u)}+p_{(v)}\right\}-\varepsilon^{\alpha \beta} r_{\alpha}\left\{p_{(u) \beta}+p_{(v) \beta}\right\} k \cdot r \\
= & -\frac{1}{2}(k \cdot r)^{2} \varepsilon_{\alpha \beta}\left\{\frac{p_{(u)}^{\alpha} p_{(u)}^{\beta}}{k \cdot p_{(u)}}+\frac{p_{(v)}^{\alpha} p_{(v)}^{\beta}}{k \cdot p_{(v)}}\right\}+\frac{1}{2}(k \cdot r)^{2} \varepsilon_{\alpha \beta} \frac{\left\{p_{(u)}^{\alpha}+p_{(v)}^{\alpha}\right\}\left\{p_{(u)}^{\beta}+p_{(v)}^{\beta}\right\}}{k \cdot\left\{p_{(u)}+p_{(v)}\right\}} .
\end{aligned}
$$

We shall now compare this with the difference in $\bar{S}_{\mathrm{gr}}^{(2)}$, given in the second line of (7.52), between the first and the second configurations. Since for $r_{(u)}=r_{(v)}=r$ the two diagrams have the same set of lines, the difference comes solely from the terms proportional to $\left(k \cdot r_{(a)}\right)^{2}$.This gives

$$
\Delta \bar{S}_{\mathrm{gr}}^{(2)}=-\frac{1}{2}(k \cdot r)^{2} \varepsilon_{\alpha \beta}\left\{\frac{p_{(u)}^{\alpha} p_{(u)}^{\beta}}{k \cdot p_{(u)}}+\frac{p_{(v)}^{\alpha} p_{(v)}^{\beta}}{k \cdot p_{(v)}}\right\}+\frac{1}{2}(k \cdot r)^{2} \varepsilon_{\alpha \beta} \frac{\left\{p_{(u)}^{\alpha}+p_{(v)}^{\alpha}\right\}\left\{p_{(u)}^{\beta}+p_{(v)}^{\beta}\right\}}{k \cdot\left\{p_{(u)}+p_{(v)}\right\}} .
$$

Comparing (7.55) and (7.56) we see that $\Delta S_{\mathrm{gr}}^{(2)}=\Delta \bar{S}_{\mathrm{gr}}^{(2)}$. Since we have assumed that for tree diagrams with $n$ external states $S_{\mathrm{gr}}^{(2)}=\bar{S}_{\mathrm{gr}}^{(2)}$, this establishes the equality of $S_{\mathrm{gr}}^{(2)}$ and $\bar{S}_{\mathrm{gr}}^{(2)}$ for tree diagrams with $n+1$ external states. ${ }^{7}$ Repeating this analysis we can establish the equality of $S_{\mathrm{gr}}^{(2)}$ and $\bar{S}_{\mathrm{gr}}^{(2)}$ for all tree diagrams.

We now turn to the second step - the loop diagrams. Our starting assumption is that the equality of $\bar{S}_{\mathrm{gr}}^{(2)}$ and $S_{\mathrm{gr}}^{(2)}$ holds for all diagrams with $\ell$ or less loops. Let us consider a diagram with $\ell+1$ loops. We can get from this a diagrams with $\ell$ loops by picking an internal line that forms part of a loop and regarding this as a pair of external lines carrying opposite momenta. If the internal line extends from $r_{1}$ to $r_{2}$ and carries momentum $p$, then

\footnotetext{
${ }^{7}$ In making this inference we have assumed that the tree diagram with $(n+1)$ external states has at least two external lines that join to an internal line via a three point vertex. One may worry that this proof does not hold for diagrams in which all external states connect to vertices with more than three legs. This can be addressed by noting that a vertex with more than three legs can be realized by starting with a diagram containing only three point vertices, and then taking the lengths of some of the internal lines to zero.
} 
the difference in $S_{\mathrm{gr}}^{(2)}$ between the first diagram with $\ell+1$ loops and the second diagram with $\ell$ loops comes from the two extra external lines that the second diagram carries. As before in the second diagram we shall take the external lines with momenta $\pm p$ to have zero length. This gives

$$
\begin{aligned}
\Delta S_{\mathrm{gr}}^{(2)}= & \frac{1}{2} \frac{1}{k \cdot p} \varepsilon^{\alpha \beta}\left[p_{\alpha} p_{\beta}\left\{\left(k \cdot r_{2}\right)^{2}-\left(k \cdot r_{1}\right)^{2}\right\}+(k \cdot p)^{2}\left\{r_{2 \alpha} r_{2 \beta}-r_{1 \alpha} r_{1 \beta}\right\}\right. \\
& \left.-2 k \cdot p p_{\alpha}\left\{k \cdot r_{2} r_{2 \beta}-k \cdot r_{1} r_{1 \beta}\right\}\right] .
\end{aligned}
$$

On the other hand the difference in the contribution to $\bar{S}_{\mathrm{gr}}^{(2)}$ from the first and the second diagrams comes from two sources. The first diagram will have an extra line carrying momentum $p$ and extending from $r_{1}$ to $r_{2}$ and will therefore receive a contribution from that line. On the other hand the second diagram will have two additional external lines carrying momenta $\pm p$ and will therefore receive additional contribution from the terms proportional to $\left(k \cdot r_{(a)}\right)^{2}$ in $(7.52)$. This gives

$$
\begin{aligned}
\Delta \bar{S}_{\mathrm{gr}}^{(2)}= & -\frac{1}{2} \varepsilon^{\alpha \beta} p_{\alpha}\left\{k \cdot r_{2} r_{2 \beta}-k \cdot r_{1} r_{1 \beta}-k \cdot r_{2} r_{1 \beta}+k \cdot r_{1} r_{2 \beta}\right\} \\
& +\frac{1}{2} \varepsilon^{\alpha \beta} \frac{p_{\alpha} p_{\beta}}{k \cdot p}\left\{\left(k \cdot r_{2}\right)^{2}-\left(k \cdot r_{1}\right)^{2}\right\}
\end{aligned}
$$

Now using that fact that the vector $r_{2}-r_{1}$ is proportional to $p$, we can write

$$
\begin{aligned}
& p_{\alpha} k \cdot r_{2} r_{1 \beta}=p_{\alpha} k \cdot\left(r_{2}-r_{1}\right) r_{1 \beta}+p_{\alpha} k . r_{1} r_{1 \beta}=k \cdot p\left(r_{2}-r_{1}\right)_{\alpha} r_{1 \beta}+p_{\alpha} k \cdot r_{1} r_{1 \beta}, \\
& p_{\alpha} k \cdot r_{1} r_{2 \beta}=p_{\alpha} k \cdot\left(r_{1}-r_{2}\right) r_{2 \beta}+p_{\alpha} k . r_{2} r_{2 \beta}=k \cdot p\left(r_{1}-r_{2}\right)_{\alpha} r_{2 \beta}+p_{\alpha} k \cdot r_{2} r_{2 \beta} .
\end{aligned}
$$

Using this we can express (7.58) as

$$
\begin{aligned}
\Delta \bar{S}_{\mathrm{gr}}^{(2)}= & -\frac{1}{2} \varepsilon^{\alpha \beta} p_{\alpha}\left\{2 k . r_{2} r_{2 \beta}-2 k . r_{1} r_{1 \beta}\right\}+\frac{1}{2} \varepsilon^{\alpha \beta} k \cdot p\left\{\left(r_{2}-r_{1}\right)_{\alpha} r_{1 \beta}+\left(r_{2}-r_{1}\right)_{\alpha} r_{2 \beta}\right\} \\
& +\frac{1}{2} \varepsilon^{\alpha \beta} \frac{p_{\alpha} p_{\beta}}{k \cdot p}\left\{\left(k \cdot r_{2}\right)^{2}-\left(k . r_{1}\right)^{2}\right\} .
\end{aligned}
$$

Since $\varepsilon^{\alpha \beta}$ is symmetric, (7.60) and (7.57) are identical. Using the fact that for $\ell$-loop diagrams $\bar{S}_{\mathrm{gr}}^{(2)}$ and $S_{\mathrm{gr}}^{(2)}$ are identical, this establishes that for the $(\ell+1)$-loop diagrams also the two are identical. By induction, this establishes the equality of $S_{\mathrm{gr}}^{(2)}$ and $\bar{S}_{\mathrm{gr}}^{(2)}$ for all diagrams.

\subsubsection{Inclusion of spin}

In the analysis of the previous two subsections we have not included any contribution from the intrinsic spin of the objects. In this subsection we shall rectify this situation. For this we use the fact that if $\Sigma^{\alpha \beta}(\sigma)$ denotes the spin angular momentum of an object moving along the trajectory $r(\sigma)$, then its contribution to the stress tensor is given by (see e.g. [100] for recent review):

$$
\Delta T^{\alpha \beta}(x)=\int d \sigma \frac{d r^{(\alpha}(\sigma)}{d \sigma} \Sigma^{\beta) \gamma}(\sigma) \partial_{\gamma} \delta^{(D)}(x-r(\sigma)) .
$$


Its contribution to the metric is given by

$$
\begin{aligned}
\Delta h_{\alpha \beta}(x) & =\Delta e_{\alpha \beta}(x)-\frac{1}{D-2} \eta_{\alpha \beta} \Delta e_{\gamma}^{\gamma}(x), \\
\Delta e_{\alpha \beta}(x) & \equiv-\int d^{D} x^{\prime} G_{r}\left(x, x^{\prime}\right) \Delta T_{\alpha \beta}\left(x^{\prime}\right) .
\end{aligned}
$$

This can be easily evaluated following the procedure described in sections 7.1, 7.2.1, 7.2.2. Let us consider the general process described in section 7.2.2. Then the radiative component of the time Fourier transform of $\Delta e_{\alpha \beta}$, denoted by $\Delta \tilde{e}_{\alpha \beta}$, is obtained by replacing the $P_{\alpha}(\sigma) V_{\beta}(\sigma)$ factor in $(7.29)$ by $-i k^{\gamma} V_{(\alpha}(\sigma) \Sigma_{\beta) \gamma}(\sigma)$. This gives

$$
\begin{aligned}
\Delta \tilde{e}_{\alpha \beta}= & \mathcal{N} e^{i \omega R} \sum_{s} \int d \sigma_{s} e^{i \omega\left\{r^{0}\left(\sigma_{s}\right)-\hat{n} . \vec{r}\left(\sigma_{s}\right)\right\}} k^{\gamma} V_{(\alpha}\left(\sigma_{s}\right) \Sigma_{\beta) \gamma}\left(\sigma_{s}\right) \\
& +\mathcal{N} e^{i \omega R} \frac{1}{\omega}\left[e^{i \omega\left\{r^{0}(\sigma)-\hat{n} . \vec{r}(\sigma)\right\}}\left\{\frac{i k^{\gamma} V_{(\alpha}(\sigma) \Sigma_{\beta) \gamma}(\sigma)}{V^{0}(\sigma)-\hat{n} \cdot \vec{V}(\sigma)}\right\}\right]_{\text {boundary }}
\end{aligned}
$$

Here the sum over $s$ in the first term runs over all the trajectory segments, whereas the boundary terms receive contribution from the outer ends of all the external lines. Due to the explicit $k^{\gamma}$ factor, the first term begins contributing at the subsubleading order, whereas the second term begins contributing at the subleading order. Therefore, in evaluating this expression to the subsubleading order we can replace the $e^{i \omega\left\{r^{0}\left(\sigma_{s}\right)-\hat{n} . \vec{r}\left(\sigma_{s}\right)\right\}}$ factor in the first term by 1 . Since $\Sigma_{\beta \gamma}\left(\sigma_{s}\right)$ remains constant along a given trajectory segment and $V^{\alpha}\left(\sigma_{s}\right)=d r^{\alpha}\left(\sigma_{s}\right) / d \sigma_{s}$, we get

$$
\sum_{s} \int d \sigma_{s} V_{\alpha}\left(\sigma_{s}\right) \Sigma_{\beta \gamma}\left(\sigma_{s}\right)=\sum_{s}\left(J_{s}-I_{s}\right)_{\alpha} \Sigma_{\beta \gamma}\left(\sigma_{s}\right)
$$

where, as in section 7.2.2, we denote by $I_{s}$ and $J_{s}$ the beginning and the end points of the $s$-th trajectory segment. We can now rearrange the sum by first summing over all lines that enter a vertex and then summing over vertices. Since by conservation of angular momentum the sum over all $\Sigma_{\alpha \beta}$ entering a vertex vanishes, (7.65) receives contribution only from the outer ends of the external lines situated at $r_{(a)}$ for $1 \leq a \leq n$. This gives the contribution from the first term on the right hand side of (7.64) to be

$$
-\frac{1}{2} \mathcal{N} e^{i \omega R} k^{\gamma} \sum_{a=1}^{n}\left\{r_{(a) \alpha} \Sigma_{(a) \beta \gamma}+r_{(a) \beta} \Sigma_{(a) \alpha \gamma}\right\} .
$$

On the other hand, the contribution from the second term on the right hand side of (7.64) to this order is given by

$$
-\frac{1}{2} i \mathcal{N} e^{i \omega R} \sum_{a=1}^{n}\left(1+i k \cdot r_{(a)}\right) \frac{1}{k \cdot p_{(a)}} k^{\gamma}\left\{p_{(a) \alpha} \Sigma_{(a) \beta \gamma}+p_{(a) \beta} \Sigma_{(a) \alpha \gamma}\right\} .
$$

Adding (7.66) and (7.67) and contracting the result with the polarization tensor $\varepsilon^{\alpha \beta}$, we get

$$
\varepsilon^{\alpha \beta} \Delta \tilde{e}_{\alpha \beta}=\mathcal{N} e^{i \omega R} \Delta \bar{S}_{\mathrm{gr}}
$$


where

$$
\Delta \bar{S}_{\mathrm{gr}}=-i \sum_{a=1}^{n} \frac{1}{k \cdot p_{(a)}} \varepsilon^{\alpha \beta} k^{\gamma} p_{(a) \alpha} \Sigma_{(a) \beta \gamma}+\sum_{a=1}^{n} \frac{1}{k \cdot p_{(a)}} \varepsilon^{\alpha \beta} k^{\gamma}\left\{k \cdot r_{(a)} p_{(a) \alpha}-k \cdot p_{(a)} r_{(a) \alpha}\right\} \Sigma_{(a) \beta \gamma} .
$$

We shall now compare this to the change in the soft factor when we replace $\mathbf{J}_{(a)}^{\mu \nu}$ in (7.53) by

$$
\mathbf{J}_{(a)}^{\mu \nu}=r_{(a)}^{\mu} p_{(a)}^{\nu}-r_{(a)}^{\nu} p_{(a)}^{\mu}+\Sigma_{(a)}^{\mu \nu},
$$

and collect terms that are linear in $\Sigma^{\mu \nu}$. Using (3.6) and (6.5), we get

$$
\Delta S_{\mathrm{gr}}=i \sum_{a=1}^{n} \frac{\varepsilon_{\mu \nu} p_{(a)}^{\mu} k_{\rho}}{p_{(a)} \cdot k} \Sigma_{(a)}^{\rho \nu}-\sum_{a=1}^{n}\left(p_{(a)} \cdot k\right)^{-1} \varepsilon_{\mu \rho} k_{\nu} k_{\sigma}\left\{r_{(a)}^{\mu} p_{(a)}^{\nu}-r_{(a)}^{\nu} p_{(a)}^{\mu}\right\} \Sigma_{(a)}^{\rho \sigma} .
$$

Using the antisymmetry of $\Sigma_{(a) \beta \gamma}$ one can easily verify that (7.69) and (7.71) are equal. This establishes the validity of the soft theorem to subsubleading order for terms linear in $\Sigma_{(a) \beta \gamma}$. As already argued before, we do not expect this relation to hold for terms quadratic in the spin, since their contribution is of the same order as the non-universal terms.

\subsubsection{Probe scattering from a charged scatterer}

The examples in sections 7.2.1 and 7.2.2 involve scattering in which the stress tensor the source of gravity wave - is localized on a one dimensional subspace of space-time. We shall now consider the case of scattering of a probe of charge $q$ by a large mass scatterer of charge $Q$. We shall assume that the probe is sufficiently light so that we can ignore the gravitational force compared to the electromagnetic force. We shall furthermore assume that there are two $\mathrm{U}(1)$ gauge fields, one of them with wrong sign kinetic term, and that the charges $q$ and $Q$ are both null vectors in this two dimensional charge space so that $q^{2}$ and $Q^{2}$ vanish but $q \cdot Q \neq 0$. Such a theory of course is not fully consistent, but it can be regarded as a toy model for checking the soft graviton theorem. In this case the stress tensor for the fields produced by either the probe or the scatterer vanish, but the cross term survives. We emphasize that while this choice of charges simplifies our analysis, we expect soft theorem to work even without these assumptions.

We shall now compute the radiative component of the gravitational field during the scattering to subsubleading order in the soft momentum. It follows from the same analysis that led to (7.27), (7.28) that we have

$$
e_{\alpha \beta}(x)=-\int d^{D} x^{\prime} G_{r}\left(x, x^{\prime}\right) T_{\alpha \beta}\left(x^{\prime}\right)
$$

where $T_{\alpha \beta}$ is the total stress tensor. We shall first focus on the computation of the spatial components $e_{i j}$ for $1 \leq i, j \leq(D-1)$ for which the source is $-T_{i j}$. The contribution to $T_{i j}$ due to the scatterer vanishes in the infinite mass limit so that $T_{i j}$ is given by a sum of two terms - one due to the probe and the other due to the stress energy tensor of the electromagnetic field. We shall denote the corresponding contributions to $e_{i j}$ by $e_{i j}^{1}$ and $e_{i j}^{2}$ respectively. The time Fourier transform of $e_{i j}^{1}$, denoted by $\tilde{e}_{i j}^{1}$, is given by the same 
formula as (7.43) where the sum over $a$ now runs over only the initial and the final state of the probe:

$$
\tilde{e}_{i j}^{1}=\mathcal{N} e^{i \omega R} \sum_{a=1}^{2} e^{i k \cdot r_{(a)}} \frac{p_{(a) i} p_{(a) j}}{k \cdot p_{(a)}}+i \mathcal{N} e^{i \omega R} \int d \sigma e^{i k \cdot r(\sigma)} P_{i} V_{j} .
$$

Here the integral over $\sigma$ runs over the whole trajectory from $r(\sigma)=r_{(1)}$ to $r(\sigma)=r_{(2)}$. We shall expand $e^{i k \cdot r(\sigma)}$ in power series in $k$ and work up to subsubleading order. After writing $V=d r / d \sigma$, integrating the second term by parts and taking into account the fact that for the outgoing particle $P_{(2)}=-p_{(2)}$, we get, up to subsubleading order in the expansion in powers of $k$,

$$
\begin{aligned}
\tilde{e}_{i j}^{1}=\mathcal{N} e^{i \omega R} \sum_{a=1}^{2}[ & \left\{1+i k \cdot r_{(a)}-\frac{1}{2}\left(k \cdot r_{(a)}\right)^{2}\right\} \frac{p_{(a) i} p_{(a) j}}{k \cdot p_{(a)}} \\
& -\frac{i}{2} \sum_{a=1}^{2}\left\{p_{(a) i} r_{(a) j}+p_{(a) j} r_{(a) i}\right\}\left(1+i k \cdot r_{(a)}\right) \\
& -\frac{i}{2} \int d \sigma\left\{\frac{d P_{i}}{d \sigma} r_{j}(\sigma)+\frac{d P_{j}}{d \sigma} r_{i}(\sigma)\right\}\{1+i k \cdot r(\sigma)\} \\
& \left.+\frac{1}{2} \int d \sigma k \cdot V(\sigma)\left\{P_{i} r_{j}+P_{j} r_{i}\right\}\right] .
\end{aligned}
$$

Now using the fact that $P$ and $V$ are proportional to each other, the last term may be manipulated as

$$
\begin{aligned}
\frac{1}{2} \int d \sigma k \cdot V(\sigma)\left\{P_{i} r_{j}+P_{j} r_{i}\right\} & =\frac{1}{2} \int d \sigma k \cdot P(\sigma)\left\{V_{i} r_{j}+V_{j} r_{i}\right\} \\
& =\frac{1}{2} \int d \sigma k \cdot P(\sigma) \frac{d}{d \sigma}\left\{r_{i} r_{j}\right\} \\
& =-\frac{1}{2} \sum_{a=1}^{2} k \cdot p_{(a)} r_{(a) i} r_{(a) j}-\frac{1}{2} \int d \sigma k \cdot \frac{d P(\sigma)}{d \sigma}\left\{r_{i} r_{j}\right\}
\end{aligned}
$$

where in the last step we have carried out an integration by parts. With this (7.74) can be written as

$$
\begin{aligned}
\tilde{e}_{i j}^{1}= & \mathcal{N} e^{i \omega R} \sum_{a=1}^{2}\left[\left\{1+i k \cdot r_{(a)}-\frac{1}{2}\left(k \cdot r_{(a)}\right)^{2}\right\} \frac{p_{(a) i} p_{(a) j}}{k \cdot p_{(a)}}\right. \\
& -\frac{i}{2} \sum_{a=1}^{2}\left\{p_{(a) i} r_{(a) j}+p_{(a) j} r_{(a) i}\right\}\left(1+i k \cdot r_{(a)}\right)-\frac{1}{2} \sum_{a=1}^{2} k \cdot p_{(a)} r_{(a) i} r_{(a) j} \\
& \left.-\frac{i}{2} \int d \sigma\left\{\frac{d P_{i}}{d \sigma} r_{j}(\sigma)+\frac{d P_{j}}{d \sigma} r_{i}(\sigma)\right\}\{1+i k \cdot r(\sigma)\}-\frac{1}{2} \int d \sigma k \cdot \frac{d P(\sigma)}{d \sigma}\left\{r_{i}(\sigma) r_{j}(\sigma)\right\}\right] .
\end{aligned}
$$

On the other hand, $e_{i j}^{2}$ is given by

$$
e_{i j}^{2}=-\int d^{D} x^{\prime} G_{r}\left(x, x^{\prime}\right)\left[F_{i \rho}\left(x^{\prime}\right) \cdot F_{j}{ }^{\rho}\left(x^{\prime}\right)-\frac{1}{4} \delta_{i j} F_{\rho \sigma}\left(x^{\prime}\right) \cdot F^{\rho \sigma}\left(x^{\prime}\right)\right],
$$


where denotes the inner product in the two dimensional space labelling the two $\mathrm{U}(1)$ gauge fields. Manipulating this expression in the same way that led from (7.3) to (7.10) we see that the radiative part of (7.77) is given by

$$
\tilde{e}_{i j}^{2}=i \mathcal{N} e^{i \omega R} \int d^{D} x^{\prime} e^{i k \cdot x^{\prime}}\left[F_{i \rho}\left(x^{\prime}\right) \cdot F_{j}{ }^{\rho}\left(x^{\prime}\right)-\frac{1}{4} \delta_{i j} F_{\rho \sigma}\left(x^{\prime}\right) \cdot F^{\rho \sigma}\left(x^{\prime}\right)\right] .
$$

Now $F_{\mu \nu}$ can be written as the sum of the gauge field strength $F_{\mu \nu}^{S}$ produced by the scatterer and the gauge field strength $F_{\mu \nu}^{P}$ produced by the probe. Using the fact that the charges carried by the probe and the scatterers are null, we can express (7.78) as

$$
\tilde{e}_{i j}^{2}=i \mathcal{N} e^{i \omega R} \int d^{D} x^{\prime} e^{i k \cdot x^{\prime}}\left[F_{i \rho}^{P}\left(x^{\prime}\right) \cdot F^{S}{ }_{j}^{\rho}\left(x^{\prime}\right)+F_{i \rho}^{S}\left(x^{\prime}\right) \cdot F_{j}^{P}{ }_{j}^{\rho}\left(x^{\prime}\right)-\frac{1}{2} \delta_{i j} F_{\rho \sigma}^{S}\left(x^{\prime}\right) \cdot F^{P \rho \sigma}\left(x^{\prime}\right)\right] \text {. }
$$

Now the prediction of soft theorem for $\tilde{e}_{i j}$ according to (1.2)-(1.5) (with $\mathbf{J}_{i j}=0$ ) is

$$
\begin{gathered}
\mathcal{N} e^{i \omega R}\left[\sum_{a=1}^{2} \frac{p_{(a) i} p_{(a) j}}{p_{(a)} \cdot k}+\frac{i}{2} \sum_{a=1}^{2} \frac{1}{p_{(a)} \cdot k}\left\{p_{(a) i} k_{\rho}\left(\mathbf{j}_{(a)}\right)_{j}^{\rho}+p_{(a) j} k_{\rho}\left(\mathbf{j}_{(a)}\right)_{i}^{\rho}\right\}\right. \\
\left.-\frac{1}{2} \sum_{a=1}^{2}\left(p_{(a)} \cdot k\right)^{-1} \varepsilon_{i j} k_{\nu} k_{\sigma} \mathbf{j}_{(a)}^{i \nu} \mathbf{j}_{(a)}^{j \sigma}\right] .
\end{gathered}
$$

Using the result $\mathbf{j}_{(a)}^{\mu \nu}=r_{(a)}^{\mu} p_{(a)}^{\nu}-r_{(a)}^{\nu} p_{(a)}^{\mu}$ it is easy to check that the terms in the first two lines on the right hand side of (7.76) produce the desired contribution (7.80). Therefore we need to show that the sum of (7.79) and the term in the third line of the right hand side of (7.76) vanishes.

Let us denote by $I_{i j}$ the contribution to $\tilde{e}_{i j}^{1}$ from the third line of the right hand side of (7.76). Using the equations of motion of the probe ${ }^{8}$

$$
\frac{d P_{\alpha}}{d \sigma}=q \cdot F_{\alpha \rho}^{S}(r(\sigma)) \frac{d r^{\rho}}{d \sigma},
$$

and the fact that the only non-vanishing components of the Coulomb field of the scatterer are $F_{i 0}^{S}=-F_{0 i}^{S}$, we can express $I_{i j}$ as

$$
\begin{aligned}
I_{i j}= & -\frac{i}{2} \mathcal{N} e^{i \omega R} \int d \sigma\left\{q \cdot F_{i 0}^{S}(r(\sigma)) \frac{d r^{0}}{d \sigma} r_{j}(\sigma)+q \cdot F_{j 0}^{S}(r(\sigma)) \frac{d r^{0}}{d \sigma} r_{i}(\sigma)\right\}\{1+i k \cdot r(\sigma)\} \\
& -\frac{1}{2} \mathcal{N} e^{i \omega R} \int d \sigma\left\{k^{0} q \cdot F_{0 m}^{S}(r(\sigma)) \frac{d r^{m}}{d \sigma}+k^{m} q \cdot F_{m 0}^{S}(r(\sigma)) \frac{d r^{0}}{d \sigma}\right\}\left\{r_{i}(\sigma) r_{j}(\sigma)\right\} .
\end{aligned}
$$

Let us denote by $\widetilde{F}_{\mu \nu}(\ell)$ the Fourier transform of $F_{\mu \nu}$ in all variables:

$$
\widetilde{F}_{\mu \nu}(\ell)=\int d^{D} x e^{-i \ell \cdot x} F_{\mu \nu}(x) .
$$

Then (7.82) may be expressed as

$$
\begin{aligned}
I_{i j}= & -\frac{i}{2} \mathcal{N} e^{i \omega R} \int d \sigma \int \frac{d^{D} \ell}{(2 \pi)^{D}} e^{i \ell \cdot r(\sigma)} \frac{d r^{0}}{d \sigma}\left\{q \cdot \widetilde{F}_{i 0}^{S}(\ell) r_{j}(\sigma)+q \cdot \widetilde{F}_{j 0}^{S}(\ell) r_{i}(\sigma)\right\}\{1+i k \cdot r(\sigma)\} \\
& -\frac{1}{2} \mathcal{N} e^{i \omega R} \int d \sigma \int \frac{d^{D} \ell}{(2 \pi)^{D}} e^{i \ell \cdot r(\sigma)}\left\{k^{0} q \cdot \widetilde{F}_{0 m}^{S}(\ell) \frac{d r^{m}}{d \sigma}+k^{m} q \cdot \widetilde{F}_{m 0}^{S}(\ell) \frac{d r^{0}}{d \sigma}\right\}\left\{r_{i}(\sigma) r_{j}(\sigma)\right\} .
\end{aligned}
$$

\footnotetext{
${ }^{8}$ The sign in (7.81) is convention dependent. It has been chosen so as to be consistent with (7.1).
} 
Now using the Euclidean Green's function in $(D-1)$ dimension we see that the Fourier transform of the Coulomb field $\widetilde{A}^{0}$ produced by a static source of charge $Q$ at the origin is given by

$$
\widetilde{A}^{0}=\frac{Q}{\overrightarrow{\ell^{2}}} 2 \pi \delta\left(\ell^{0}\right)
$$

leading to

$$
\widetilde{F}_{i 0}^{S}(\ell)=-i \ell_{i} Q \frac{1}{\vec{\ell}^{2}} 2 \pi \delta\left(\ell^{0}\right) .
$$

Substituting (7.86) into (7.84) we get

$$
\begin{aligned}
I_{i j}= & \frac{i}{2} \mathcal{N} e^{i \omega R} \int d \sigma \int \frac{d^{D-1} \ell}{(2 \pi)^{D-1}} e^{i \vec{\ell} \cdot \vec{r}(\sigma)} \frac{1}{\overrightarrow{\ell^{2}}} q \cdot Q \frac{d r^{0}}{d \sigma}\left\{i \ell_{i} r_{j}(\sigma)+i \ell_{j} r_{i}(\sigma)\right\}\{1+i k \cdot r(\sigma)\} \\
& +\frac{i}{2} \mathcal{N} e^{i \omega R} \int d \sigma \int \frac{d^{D-1} \ell}{(2 \pi)^{D-1}} e^{i \vec{\ell} \cdot \vec{r}(\sigma)} \frac{1}{\overrightarrow{\ell^{2}}} q \cdot Q\left\{-k^{0} \ell_{m} \frac{d r^{m}}{d \sigma}+\vec{k} \cdot \vec{\ell} \frac{d r^{0}}{d \sigma}\right\}\left\{r_{i}(\sigma) r_{j}(\sigma)\right\}
\end{aligned}
$$

We now turn to the analysis of $\tilde{e}_{i j}^{2}$ given in (7.79). We have

$$
\tilde{e}_{i j}^{2}=i \mathcal{N} e^{i \omega R} \int d^{D} x^{\prime} e^{i k \cdot x^{\prime}}\left[-F_{i 0}^{P}\left(x^{\prime}\right) \cdot F_{j 0}^{S}\left(x^{\prime}\right)-F_{j 0}^{P}\left(x^{\prime}\right) \cdot F_{i 0}^{S}\left(x^{\prime}\right)+\delta_{i j} F_{k 0}^{S}\left(x^{\prime}\right) \cdot F_{k 0}^{P}\left(x^{\prime}\right)\right]
$$

It follows from $(7.3),(7.4)$ that the Fourier transform $\widetilde{F}_{i 0}^{P}$ of the field generated by the probe is given by

$$
\widetilde{F}_{i 0}^{P}(-\ell-k)=-q \frac{1}{\left(\ell^{0}+k^{0}-i \epsilon\right)^{2}-(\vec{\ell}+\vec{k})^{2}} \int d \sigma e^{i(\ell+k) \cdot r(\sigma)}\left\{-i\left(\ell_{i}+k_{i}\right) \frac{d r_{0}}{d \sigma}+i\left(\ell_{0}+k_{0}\right) \frac{d r_{i}}{d \sigma}\right\} .
$$

Using (7.83), (7.86), (7.89), $\tilde{e}_{i j}^{2}$ given in (7.88) may be expressed as (to subsubleading order)

$$
\begin{aligned}
\tilde{e}_{i j}^{2}= & i \mathcal{N} e^{i \omega R} \int \frac{d^{D} \ell}{(2 \pi)^{D}}\left[y-\widetilde{F}_{i 0}^{P}(-\ell-k) \cdot \widetilde{F}_{j 0}^{S}(\ell)-\widetilde{F}_{j 0}^{P}(-\ell-k) \cdot \widetilde{F}_{i 0}^{S}(\ell)+\delta_{i j} \widetilde{F}_{k 0}^{P}(-\ell-k) \cdot \widetilde{F}_{k 0}^{S}(\ell)\right] \\
= & i \mathcal{N} e^{i \omega R} \int d \sigma \int \frac{d^{D-1} \ell}{(2 \pi)^{D-1}} e^{i \vec{\ell} \cdot \vec{r}(\sigma)+i k \cdot r(\sigma)} q \cdot Q \frac{1}{\left(\overrightarrow{\ell^{2}}\right)\left(\overrightarrow{\ell^{2}}+2 \vec{\ell} \cdot \vec{k}\right)} \\
& {\left[\left\{2 \ell_{i} \ell_{j}+\ell_{i} k_{j}+\ell_{j} k_{i}-\left(\vec{\ell}^{2}+\vec{\ell} \cdot \vec{k}\right) \delta_{i j}\right\} \frac{d r_{0}}{d \sigma}+\left\{-k_{0} \ell_{j} \frac{d r_{i}}{d \sigma}-k_{0} \ell_{i} \frac{d r_{j}}{d \sigma}+k_{0} \ell_{m} \frac{d r_{m}}{d \sigma} \delta_{i j}\right\}\right] } \\
= & i \mathcal{N} e^{i \omega R} \int d \sigma \int \frac{d^{D-1} \ell}{(2 \pi)^{D-1}} e^{i \vec{\ell} \cdot \vec{r}(\sigma)} q \cdot Q \frac{1}{\left(\overrightarrow{\ell^{2}}\right)^{2}}\left\{2 \ell_{i} \ell_{j}-\vec{\ell}^{2} \delta_{i j}\right\} \frac{d r_{0}}{d \sigma}\{1+i k \cdot r(\sigma)\} \\
& +i \mathcal{N} e^{i \omega R} \int d \sigma \int \frac{d^{D-1} \ell}{(2 \pi)^{D-1}} e^{i \vec{\ell} \cdot \vec{r}(\sigma)} q \cdot Q \frac{1}{\left(\vec{\ell}^{2}\right)^{3}}\left\{-4 \ell_{i} \ell_{j} \vec{\ell} \cdot \vec{k}+\vec{\ell}^{2}\left(\ell_{i} k_{j}+\ell_{j} k_{i}\right)+\vec{\ell} \cdot \vec{k} \vec{\ell}^{2} \delta_{i j}\right\} \frac{d r_{0}}{d \sigma} \\
& +i \mathcal{N} e^{i \omega R} \int d \sigma \int \frac{d^{D-1} \ell}{(2 \pi)^{D-1}} e^{i \vec{\ell} \cdot \vec{r}(\sigma)} q \cdot Q \frac{1}{\left(\overrightarrow{\ell^{2}}\right)^{2}}\left\{-k_{0} \ell_{j} \frac{d r_{i}}{d \sigma}-k_{0} \ell_{i} \frac{d r_{j}}{d \sigma}+k_{0} \ell_{m} \frac{d r_{m}}{d \sigma} \delta_{i j}\right\} .
\end{aligned}
$$


The integrand of the last term proportional to $\delta_{i j}$ is proportional to $\frac{d}{d \sigma} e^{i \vec{\ell} \cdot \vec{r}(\sigma)}$ and vanishes by integration by parts. In this case there is no boundary terms since the $e^{i \vec{\ell} \cdot \vec{r}(\sigma)}$ is oscillatory and as a result the boundary terms vanish after integration over $\vec{\ell}$. We now use the relations

$$
\begin{aligned}
\frac{1}{\left(\overrightarrow{\ell^{2}}\right)^{2}}\left\{2 \ell_{i} \ell_{j}-\vec{\ell}^{2} \delta_{i j}\right\} & =-\frac{1}{2}\left[\frac{\partial}{\partial \ell_{i}}\left(\frac{\ell_{j}}{\overrightarrow{\ell^{2}}}\right)+\frac{\partial}{\partial \ell_{j}}\left(\frac{\ell_{i}}{\overrightarrow{\ell^{2}}}\right)\right], \quad \frac{\ell_{m}}{\left(\overrightarrow{\ell^{2}}\right)^{2}}=-\frac{1}{2} \frac{\partial}{\partial \ell_{m}}\left(\frac{1}{\overrightarrow{\ell^{2}}}\right), \\
\frac{1}{2} \frac{\partial^{2}}{\partial \ell_{i} \partial \ell_{j}}\left(\frac{\vec{\ell} \cdot \vec{k}}{\overrightarrow{\ell^{2}}}\right) & =\frac{1}{\left(\overrightarrow{\ell^{2}}\right)^{3}}\left\{4 \ell_{i} \ell_{j} \vec{\ell} \cdot \vec{k}-\left(k_{i} \ell_{j}+k_{j} \ell_{i}\right) \vec{\ell}^{2}-\delta_{i j} \overrightarrow{\ell^{2}} \vec{\ell} \cdot \vec{k}\right\},
\end{aligned}
$$

and integration by parts, to express (7.90) as

$$
\begin{aligned}
\tilde{e}_{i j}^{2}= & \frac{i}{2} \mathcal{N} e^{i \omega R} \int d \sigma \int \frac{d^{D-1} \ell}{(2 \pi)^{D-1}} e^{i \vec{\ell} \cdot \vec{r}(\sigma)} q \cdot Q \frac{1}{\overrightarrow{\ell^{2}}}\left\{i \ell_{i} r_{j}+i \ell_{j} r_{i}\right\} \frac{d r_{0}}{d \sigma}\{1+i k \cdot r(\sigma)\} \\
& +\frac{i}{2} \mathcal{N} e^{i \omega R} \int d \sigma \int \frac{d^{D-1} \ell}{(2 \pi)^{D-1}} e^{i \vec{\ell} \cdot \vec{r}(\sigma)} q \cdot Q \frac{1}{\overrightarrow{\ell^{2}}} r_{i}(\sigma) r_{j}(\sigma) \frac{d r_{0}}{d \sigma} \vec{\ell} \cdot \vec{k} \\
& +\frac{i}{2} \mathcal{N} e^{i \omega R} \int d \sigma \int \frac{d^{D-1} \ell}{(2 \pi)^{D-1}} e^{i \vec{\ell} \cdot \vec{r}(\sigma)} q \cdot Q \frac{1}{\overrightarrow{\ell^{2}}}\left\{-i k_{0} r_{j} \frac{d r_{i}}{d \sigma}-i k_{0} r_{i} \frac{d r_{j}}{d \sigma}\right\} .
\end{aligned}
$$

The terns in the last line can be combined into a single derivative with respect to $\sigma$. Integrating by parts over the $\sigma$ variable, we can bring (7.92) to the form

$$
\begin{aligned}
\tilde{e}_{i j}^{2}= & \frac{i}{2} \mathcal{N} e^{i \omega R} \int d \sigma \int \frac{d^{D-1} \ell}{(2 \pi)^{D-1}} e^{i \vec{\ell} \cdot \vec{r}(\sigma)} q \cdot Q \frac{1}{\vec{\ell}^{2}}\left\{i \ell_{i} r_{j}+i \ell_{j} r_{i}\right\} \frac{d r_{0}}{d \sigma}\{1+i k \cdot r(\sigma)\} \\
& +\frac{i}{2} \mathcal{N} e^{i \omega R} \int d \sigma \int \frac{d^{D-1} \ell}{(2 \pi)^{D-1}} e^{i \vec{\ell} \cdot \vec{r}(\sigma)} q \cdot Q \frac{1}{\vec{\ell}^{2}} r_{i}(\sigma) r_{j}(\sigma) \frac{d r_{0}}{d \sigma} \vec{k} \cdot \vec{\ell} \\
& +\frac{i}{2} \mathcal{N} e^{i \omega R} \int d \sigma \int \frac{d^{D-1} \ell}{(2 \pi)^{D-1}} e^{i \vec{\ell} \cdot \vec{r}(\sigma)} q \cdot Q \frac{1}{\vec{\ell}^{2}}\left\{-k_{0} \ell_{m} \frac{d r_{m}}{d \sigma} r_{i}(\sigma) r_{j}(\sigma)\right\} .
\end{aligned}
$$

Eq. (7.87), (7.93) and the relations $r_{0}=-r^{0}, k_{0}=-k^{0}$ now give

$$
I_{i j}+\tilde{e}_{i j}^{2}=0 .
$$

This shows that all the extra terms cancel and that $\tilde{e}_{i j}$ has the correct form as given in $(7.80)$.

Now it follows from (7.72) and the conservation of the total stress tensor that $\partial^{\mu} e_{\mu \nu}=$ 0 . Using this condition we get

$$
\tilde{e}_{00}(\omega, \vec{k})=\frac{k^{i} k^{j}}{k_{0}^{2}} \tilde{e}_{i j}, \quad \tilde{e}_{0 i}(\omega, \vec{k})=-\frac{k^{j}}{k^{0}} \tilde{e}_{i j} .
$$

Using the form (7.80) for $\tilde{e}_{i j}$ we now get

$$
\varepsilon^{\mu \nu} \tilde{e}_{\mu \nu}=\mathcal{N} e^{i \omega R}\left\{S_{\mathrm{gr}}^{(0)}(\varepsilon, k)+S_{\mathrm{gr}}^{(1)}(\varepsilon, k)+S_{\mathrm{gr}}^{(2)}(\varepsilon, k)\right\},
$$

with $S_{\mathrm{gr}}^{(0)}, S_{\mathrm{gr}}^{(1)}$ and $S_{\mathrm{gr}}^{(2)}$ given by (1.2), (1.3) and (1.4) (with $\mathbf{J}_{i j}=0$ ) respectively. It now follows from the same analysis that led from (7.20) to (7.26) that the angular power 
spectrum of soft graviton radiation emitted during this process agrees with the prediction of soft graviton theorem to subsubleading order.

The next two examples will test soft theorem only to leading order, but have been included since they take into account the effect of the gravitational field, produced by the macroscopic objects involved in the scattering, on each other.

\subsubsection{Probe scattering from Schwarzschild black hole}

Ref. [101] considered the scattering of a probe particle of mass $m$ in the background geometry produced by a Schwarzschild blackhole of mass $M_{0} \gg m$ in the limit where the impact parameter $b$ is much larger then the Schwarzschild radius $a$ of the blackhole. In particular [101] gives the expression for the time Fourier transform of the radiative component of the metric fluctuation $h_{\alpha \beta}$. The computation was more sophisticated than the one given in [85] (as reported at the beginning of section 7.2.1) in that it took into account the effect of Schwarzschild geometry on the probe particle. Therefore we can in principle compare the small $\omega$ expansion of the results of [101] with our results. Unfortunately since the computation was done in four space-time dimensions, the soft expansion suffers from infrared divergences as discussed in section 8. For this reason we have to limit our comparison to the leading order term in the expansion which is not affected by infrared divergences. ${ }^{9}$

Starting point of our analysis is eq. (2.11) of [101] which gives the expression for the spatial component of $\tilde{e}_{\alpha \beta} \equiv \tilde{h}_{\alpha \beta}-\frac{1}{2} \eta_{\alpha \beta} \tilde{h}_{\rho}^{\rho}$ (called $\tilde{h}_{\alpha \beta}$ in [101]). There is a difference in the normalization factor of 2 between the our definition of $h_{\alpha \beta}$ (which takes the metric to be $\eta_{\alpha \beta}+2 h_{\alpha \beta}$ ) and the definition used in [101] (which takes the metric to be $\eta_{\alpha \beta}+h_{\alpha \beta}$ ). There is also an overall sign difference since [101] uses metric with mostly minus signature whereas we are using metric with mostly plus signature. Taking these into account and setting $8 \pi G=1$, the result of eq. (2.11), (2.12) of [101] for large $|\vec{x}|$ may be expressed as

$$
\tilde{e}_{i j}(\vec{x}, \omega)=\tilde{e}_{i j}^{(1)}(\vec{x}, \omega)+\tilde{e}_{i j}^{(2)}(\vec{x}, \omega)+\text { terms subleading in } \omega \rightarrow 0 \text { limit },
$$

where

$$
\tilde{e}_{i j}^{(1)}(\vec{x}, \omega)=\frac{m}{4 \pi(1+2 \varphi)} \int \frac{d t^{\prime}}{1+2 \varphi^{\prime}} \frac{d t^{\prime}}{d s} v_{i} v_{j} \frac{e^{i \omega\left(t^{\prime}+R^{\prime}\right)}}{R^{\prime}},
$$

and

$$
\begin{gathered}
\tilde{e}_{i j}^{(2)}(\vec{x}, \omega)=i \frac{M_{0} m}{32 \pi^{2} \omega} \frac{e^{i \omega R}}{R} \int d t^{\prime} \frac{d t^{\prime}}{d s}\left(1+\vec{v}^{2}\right)\left(\partial_{i}^{\prime} \partial_{j}^{\prime}-\frac{1}{2} \delta_{i j} \partial_{k}^{\prime} \partial_{k}^{\prime}\right)\left\{\ln \left(r^{\prime}+\hat{n} \cdot \vec{r}^{\prime}\right) e^{i \omega\left(t^{\prime}-\hat{n} \cdot \vec{r}^{\prime}\right)}\right. \\
\left.+\int_{r^{\prime}+\hat{n} \cdot \vec{r}^{\prime}}^{\infty} \frac{d u}{u} e^{i \omega\left(t^{\prime}-\hat{n} \cdot \vec{r}^{\prime}+u\right)}\right\}\left.\right|_{\vec{r}^{\prime}=\vec{x}^{\prime}\left(t^{\prime}\right)} .
\end{gathered}
$$

Here $\vec{x}^{\prime}\left(t^{\prime}\right)$ denotes the trajectory of the particle, $\varphi=-h_{00}(\vec{x}), \varphi^{\prime}=-h_{00}\left(\vec{x}^{\prime}\left(t^{\prime}\right)\right)$, s is the proper time along the trajectory, $v_{i}=d x_{i}^{\prime} / d t^{\prime}$ is the velocity along the trajectory, $R^{\prime}=\left|\vec{x}-\vec{x}^{\prime}\left(t^{\prime}\right)\right|, R=|\vec{x}|, r^{\prime}=\left|\vec{r}^{\prime}\right|$ and $\partial_{i}^{\prime} \equiv \partial / \partial r_{i}^{\prime}$. For large $|\vec{x}|=R$, we can approximate $R^{\prime}$ by $R-\hat{n} . \vec{x}^{\prime}$ where $\hat{n}$ denotes unit vector along $\vec{x}$. Also we can set $\varphi(\vec{x})$ to 0 .

\footnotetext{
${ }^{9}$ Although there are some results in higher dimensions (see e.g. [97, 102]), we do not have enough analytic results to compare them with the predictions of soft theorem.
} 
Let us first evaluate $\tilde{e}_{i j}^{(1)}$. Since as $t^{\prime} \rightarrow \pm \infty, v_{i}$ approaches a constant value, $d t^{\prime} / d s=$ $\left(1-\vec{v}^{2}\right)^{-1 / 2}$ also approaches a constant value, and $\varphi^{\prime}=-h_{00}\left(\vec{x}^{\prime}\left(t^{\prime}\right)\right)$ approaches zero, we see that the integrand in (7.98) is oscillatory. Therefore we need to define this by adding boundary terms as in going from (7.10) to (7.12). Using the fact that leading term in $\vec{x}^{\prime}\left(t^{\prime}\right)$ for large $t^{\prime}$ is $\vec{v} t^{\prime}$ we see that the required boundary term is

$$
-\left.\frac{m}{4 \pi} \frac{e^{i \omega R}}{R} \frac{v_{i} v_{j}}{\sqrt{1-\vec{v}^{2}}} \frac{1}{i \omega(1-\hat{n} \cdot \vec{v})} e^{i \omega t^{\prime}(1-\hat{n} \cdot \vec{v})}\right|_{t^{\prime}=t_{-}} ^{t^{\prime}=t_{+}} .
$$

If $p_{ \pm}$denote the momenta of the particle at $t_{ \pm}$, both counted as positive for ingoing momenta, then we have

$$
p_{ \pm}=\mp m\left(1, \vec{v}_{ \pm}\right) / \sqrt{1-\vec{v}_{ \pm}^{2}} .
$$

Also denoting by $k=-\omega(1, \hat{n})$ the momentum of the outgoing gravitons, we get

$$
k \cdot p_{ \pm}=\omega p_{ \pm}^{0}\left(1-\hat{n} \cdot \vec{v}_{ \pm}\right) .
$$

Using these results we can now extract the $1 / \omega$ term in (7.100) which is the only source of $1 / \omega$ contribution to $\tilde{e}_{i j}^{(1)}$ :

$$
\tilde{e}_{i j}^{(1)}=\frac{1}{4 \pi i R} e^{i \omega R} \sum_{a= \pm} \frac{p_{a i} p_{a j}}{k \cdot p_{a}}+\text { subleading }=\mathcal{N} e^{i \omega R} \sum_{a= \pm} \frac{p_{a i} p_{a j}}{k \cdot p_{a}}+\text { subleading }
$$

where in the second step we have used the fact that in $D=4$ the normalization factor $\mathcal{N}$ given in $(7.11)$ is $1 /(4 \pi i R)$.

Next we turn to the computation of $\tilde{e}_{i j}^{(2)}$ given in (7.99). Since the derivatives $\partial_{k}^{\prime}$ acting on the exponential factors will bring down factors of $\omega$ making the contribution subleading, we consider those terms in which the derivatives act on the other $\vec{r}^{\prime}$ dependent factors. Now we have

$$
\begin{aligned}
& \partial_{k}^{\prime}\left\{\ln \left(r^{\prime}+\hat{n} \cdot \vec{r}^{\prime}\right) e^{i \omega\left(t^{\prime}-\hat{n} \cdot \vec{r}^{\prime}\right)}+\int_{r^{\prime}+\hat{n} \cdot \vec{r}^{\prime}}^{\infty} \frac{d u}{u} e^{i \omega\left(t^{\prime}-\hat{n} \cdot \vec{r}^{\prime}+u\right)}\right\} \\
= & \left\{\partial_{k}^{\prime}\left(r^{\prime}+\hat{n} \cdot \vec{r}^{\prime}\right)\right\} \frac{1}{\left(r^{\prime}+\hat{n} \cdot \vec{r}^{\prime}\right)}\left\{e^{i \omega\left(t^{\prime}-\hat{n} \cdot \vec{r}^{\prime}\right)}-e^{i \omega\left(t^{\prime}+r^{\prime}\right)}\right\}=\mathcal{O}(\omega) .
\end{aligned}
$$

This shows that the contribution from $\tilde{e}_{i j}^{(2)}$ is subleading and $\tilde{e}_{i j}^{(1)}$ represents the full contribution to $\tilde{e}_{i j}$.

This determines the spatial components of the metric. The $e_{0 i}$ and $e_{00}$ components are determined by recalling that the constraint equations on the metric to linear order are given by $\partial^{\mu} e_{\mu \nu}=0$. In momentum space this translates to

$$
k^{\mu} \tilde{e}_{\mu \nu}=0 .
$$

We can now use the arguments given in (7.95), (7.96) to conclude that

$$
\phi(\varepsilon) \equiv \varepsilon^{\mu \nu} \tilde{e}_{\mu \nu}=\mathcal{N} e^{i \omega R} S_{\mathrm{gr}}^{(0)}(\varepsilon, k),
$$

with $S_{\mathrm{gr}}^{(0)}$ given in (1.2). The same analysis that led to the conclusion that (7.20) is consistent with the power spectrum produced according to the soft photon theorem now tells us that (7.106) is consistent with the power spectrum produced by the leading soft graviton theorem. 


\subsubsection{Scattering on the brane-world}

In [103] the authors analyzed gravitational bremsstrahlung during ultra-Planckian collision of two massive particles localized on a $d$ dimensional brane, which in turn is embedded in $D$ dimensional space-time. For definiteness we shall take the extra $(D-d)$ dimensions to be non-compact. The impact parameter $b$ is assumed to be larger than the Schrwarzchild radius associated to the center of mass energy of the particles. In this limit the deflection in the particle trajectories are small. The authors consider an iterative scheme where beginning with free inertial trajectories of the two particles localized on the brane, they compute the first order perturbation $h^{(1)}$ to the $D$-dimensional (flat) space-time metric. This is then used to compute the corrected trajectories of the particles and subsequently the correction to the corresponding stress tensors. They then compute the second order perturbations to the metric by considering the corrected stress tensor of the two particles and the stress tensor of the gravitational field $h^{(1)}$. These formulae lead them to energy spectrum of gravitational radiation in any (as opposed to low frequency) bin. To this order in perturbation theory, the authors show that it is consistent to restrict the particle trajectories to lie on the brane.

We shall show that the results of [103] to leading order in the frequency of emitted radiation are in agreement with the leading soft graviton theorem. For this we need to determine $\tilde{e}_{\alpha \beta}(\omega, \vec{x})$ to leading order in $\omega$. Now the stress tensor for the two gravitating particles is given in eq. (2.25) of [103] as

$$
T_{\alpha \beta}(x)=T_{\alpha \beta}^{(0)}(x)+T_{\alpha \beta}^{(1)}(x)
$$

where

$$
\begin{aligned}
T_{\alpha \beta}^{(0)}(x)=\sum_{i=1}^{2} \int d \sigma P_{(i) \alpha} V_{(i) \beta} \delta^{(D)}\left(x-r^{(0)}(\sigma)\right) \\
T_{\alpha \beta}^{(1)}(x)=\sum_{i=1}^{2} \int d \sigma\left[2(\delta V)_{(i)(\alpha}(\sigma) P_{(i) \beta)}+m_{(i)} \mathcal{F}_{(i) \alpha \beta}^{\mu \nu} h_{(i) \mu \nu}\left(r^{(0)}(\sigma)\right)\right. \\
\left.-P_{(i) \alpha} V_{(i) \beta} V^{(i) \mu} \partial_{\mu}\right] \delta^{(D)}\left(x-r^{(0)}(\sigma)\right)+S_{\alpha \beta} .
\end{aligned}
$$

Here $V_{(i)}$ and $P_{(i)}$ are intial velocities and momenta of the two particles, $m_{(i)}$ are their masses and $\delta V_{(i) \alpha}(\sigma)$ are the corrections to the velocities as a result of gravitational backreaction. $\mathcal{F}_{(i)}$ are tensors which depend on the initial velocities:

$$
\mathcal{F}_{(i) \alpha \beta}^{\mu \nu}=V_{(i)}^{\mu} \delta_{(\alpha}^{\nu} V_{(i) \beta)}-\frac{1}{2} \eta^{\mu \nu} V_{(i) \alpha} V_{(i) \beta},
$$

and $h_{(i)}$ gives the metric fluctuation produced at the location of the $i$-th particle due to the other particle. $S_{\alpha \beta}$ denotes the contribution to the stress tensor from the gravitational field, and the relevant part of $S_{\alpha \beta}$ that produces radiation involves the product of the gravitational fields from the two particle trajectories. As before, $\tilde{e}_{\alpha \beta}$ is computed from the stress tensor using the formula:

$$
\tilde{e}_{\alpha \beta}(\omega, \vec{x})=\int d x^{0} e^{i \omega x^{0}} \int d^{D} x^{\prime} G_{r}\left(x, x^{\prime}\right) T_{\alpha \beta}\left(x^{\prime}\right),
$$

where $G_{r}$ denotes $D$-dimensional retarded Green's function. 
Now using the fact that $V_{(i)}(\sigma)=V_{(i)}+\delta V_{(i)}(\sigma)$ and that $V_{(i)}(\sigma) \propto P_{(i)}(\sigma)$, the sum of $T_{\alpha \beta}^{(0)}(x)$ and the first term in the expression for $T_{\alpha \beta}^{(1)}(x)$ may be written as

$$
\sum_{i=1}^{2} \int d \sigma P_{(i) \alpha}(\sigma) V_{(i) \beta}(\sigma) \delta^{(D)}\left(x-r^{(0)}(\sigma)\right),
$$

up to terms of order $\left(\delta V_{(i)}(\sigma)\right)^{2}$. By standard analysis reviewed in the previous sections it follows that its contribution to the radiative part of the metric reproduces the result of the leading soft theorem up to subleading corrections.

We shall now show that the rest of the terms in $T_{\mu \nu}$ do not contribute to $\tilde{e}_{\alpha \beta}(\omega, \vec{x})$ at leading order in $\omega$. As we know from our previous analysis these terms will only contribute to leading order if the source approaches constant values as $t \rightarrow \pm \infty$. In the second term in the expression for $T_{\mu \nu}^{(1)}$, the $h_{\alpha \beta}^{(i)}(r(\sigma))$ factor, representing the gravitational field at the location of the $i$-th particle due to the other particle, vanishes as $\sigma \rightarrow \pm \infty$ since in this limit the two particles get widely separated. The same remark holds for the $S_{\mu \nu}$ term since this involves product of the gravitational fields of the two particles, in the $t \rightarrow \pm \infty$ limit this contribution vanishes. Finally the contribution to the radiative component of $\tilde{e}_{\alpha \beta}(\omega, \vec{x})$ from the third term in $T_{\alpha \beta}^{(1)}$ may be obtained by replacing the $V_{(\alpha} \Sigma_{\beta) \gamma}$ term in (7.64) by $P_{(i) \alpha} V_{(i) \beta} V_{(i) \gamma}$. We now see that due to the presence of the term proportional to $k^{\gamma}$ in (7.64), its contribution begins at the subleading order. Therefore it also does not contribute at the leading order.

\section{Modification of soft expansion in four dimensions}

In four space-time dimensions the usual S-matrix is ill defined due to infrared divergences which arise due to long range nature of the interactions, as is the case for electromagnetism and gravity. Therefore soft theorems, that give the relation between S-matrix elements, also become ill-defined except perhaps as relation between the S-matrix elements in an infra-red regulated theory [35]. The long range nature of interaction implies that scattering states or in other words asymptotic particles - are never free and their trajectories display logdivergences at late times. In this section we shall examine how infrared divergence affects classical scattering process, and soft theorem that gives the expression for soft radiation during such scattering.

We begin by examining the analysis of soft electromagnetic radiation given in section 7.1. As already mentioned, soft photon theorem holds irrespective of the nature of the scattering, e.g. even when the scatterer has no charge and the scattering takes place via non-electromagnetic interaction. However let us consider the case where the scatterer carries a charge. In this case there will be a long range Coulomb force acting on the probe even when it is far away from the scatterer. To see the effect of this we first express the late and early time straight line trajectories of the probe in the absence of long range Coulomb force, given in (7.15), as

$$
\vec{r}(t)=\vec{b}_{ \pm}+\vec{\beta}_{ \pm}\left(t-a_{ \pm}\right), \quad \text { as } t \rightarrow \pm \infty
$$


with

$$
c_{-}=\left(a_{-}, \vec{b}_{-}\right), \quad c_{+}=\left(a_{+}, \vec{b}_{+}\right), \quad V_{ \pm}=V_{ \pm}^{0}\left(1, \vec{\beta}_{ \pm}\right) .
$$

It is easy to see that in the presence of long range Coulomb force on the probe, the late and early time straight line trajectories of the probe given in (8.1) will be modified to the form

$$
\vec{r}(t)=\vec{b}_{ \pm}+\vec{\beta}_{ \pm}\left(t-a_{ \pm}\right)-C_{ \pm} \vec{\beta}_{ \pm} \ln |t|, \quad \text { as } t \rightarrow \pm \infty,
$$

where $C_{ \pm}$are constants that depend on the strength of the Coulomb interaction between the probe and the scatterer. In order to see the effect of these logarithmic terms on the soft theorem, we label $r_{ \pm}$defined in (7.13) as $\left(t_{ \pm}, \vec{r}_{ \pm}\right)$and rewrite (7.14) as

$$
\begin{aligned}
\varepsilon^{\alpha} \widetilde{A}_{\alpha}=\mathcal{N} e^{i \omega R}[ & -\frac{q \varepsilon \cdot V_{+}}{k \cdot V_{+}}\left(1-i k^{0} t_{+}+i \vec{k} \cdot \vec{r}_{+}\right)+\frac{q \varepsilon \cdot V_{-}}{k \cdot V_{-}}\left(1-i k^{0} t_{-}+i \vec{k} \cdot \vec{r}_{-}\right) \\
& \left.+i q\left(-\varepsilon^{0} t_{+}+\vec{\varepsilon} \cdot \vec{r}_{+}+\varepsilon^{0} t_{-}-\vec{\varepsilon} \cdot \vec{r}_{-}\right)\right]
\end{aligned}
$$

Substituting (8.3) into (8.4) one can easily see that the soft factor $\bar{S}_{\text {em }}$ computed from classical radiation formula, defined as the term inside the square bracket in (8.4), acquires an extra term given by

$$
\Delta \bar{S}_{\mathrm{em}}=-i q\left\{C_{+} \vec{\varepsilon} \cdot \vec{\beta}_{+} \ln \left|t_{+}\right| \frac{1}{1-\hat{n} \cdot \vec{\beta}_{+}}-C_{-} \vec{\varepsilon} \cdot \vec{\beta}_{-} \ln \left|t_{-}\right| \frac{1}{1-\hat{n} \cdot \vec{\beta}_{-}}\right\},
$$

where we have chosen $\varepsilon^{0}=0$ gauge. On the other hand the change in the form of $\vec{r}_{ \pm}(t)$ also modifies the form of $\vec{j}_{(a)}^{\mu \nu}$ given in (3.33). It is easy to see that the terms proportional to $C_{ \pm}$in (8.3) leaves $\mathbf{j}_{(a)}^{i j}$ unchanged for $1 \leq i, j \leq 3$ but changes $\mathbf{j}_{(a)}^{i 0}$ by

$$
\Delta \mathbf{j}_{ \pm}^{i 0}=\Delta r_{ \pm}^{i} p_{ \pm}^{0}=-C_{ \pm} \beta_{ \pm}^{i} p_{ \pm}^{0} \ln |t|
$$

Under this change the soft factor (6.2) computed from soft theorem changes by

$$
\Delta S_{\mathrm{em}}=-i q\left\{C_{+} \vec{\varepsilon} \cdot \vec{\beta}_{+} \ln \left|t_{+}\right| \frac{1}{1-\hat{n} \cdot \vec{\beta}_{+}}-C_{-} \vec{\varepsilon} \cdot \vec{\beta}_{-} \ln \left|t_{-}\right| \frac{1}{1-\hat{n} \cdot \vec{\beta}_{-}}\right\} .
$$

Since this is equal to (8.5) the soft theorem still holds formally. This agreement is not surprising since in both computations we effectively replace $\vec{b}_{ \pm}$by $\vec{b}_{ \pm}-C_{ \pm} \vec{\beta}_{ \pm} \ln \left|t_{ \pm}\right|$. However the presence of $\ln \left|t_{ \pm}\right|$terms in the soft factor indicates that the soft expansion itself breaks down. Instead of expanding the right hand side of (7.12) in a power series expansion in $\omega$ before integrating over $\sigma$, one should express this as

$$
-\frac{1}{\omega} \mathcal{N} e^{i \omega R} \int_{-\infty}^{\infty} d \sigma e^{i \omega\left(r^{0}(\sigma)-\hat{n} . \vec{r}(\sigma)\right)} \frac{\partial}{\partial \sigma}\left(\frac{q V_{\alpha}(\sigma)}{V^{0}(\sigma)-\hat{n} \cdot \vec{V}(\sigma)}\right),
$$

carry out the integration keeping the $\omega\left(r^{0}(\sigma)-\hat{n} \cdot \vec{r}(\sigma)\right)$ in the exponent, and then study the small $\omega$ behavior of the amplitude. The presence of the $\ln \left|t_{ \pm}\right|$term (8.7) is an indication 
that this expansion is non-analytic at $\omega=0$, involving $\ln \omega$ terms in the expansion. In the quantum theory this is reflected in the fact that derivatives of the amplitude with respect to the external momenta, hidden in the definition of $\mathcal{J}^{\mu \nu}$, diverge in the soft limit.

A similar analysis can be carried out for soft graviton theorem. Since everything gravitates, in this case it is impossible to switch off the long range interactions in the far past and far future. If for simplicity we work in the probe limit in which the particles 2,4 and 5 of section 7.2.1 are infinitely heavy, then in (7.33) we shall get terms proportional to $\ln |t|$ in the expression for $\vec{r}\left(\sigma_{1}\right)$ and $\vec{r}\left(\sigma_{3}\right)$. This will generate logarithmic corrections to the soft factor indicating that the expansion in power series in $\omega$ breaks down at the subleading order. In this case there will also be additional effects due to long range gravitational force acting on the soft gravitons.

\section{Discussion}

In this paper we have analyzed the consequences of soft theorem in the classical limit and have shown that they can predict the spectrum of long wavelength radiation during a classical scattering. We shall end by first recalling some salient features of our analysis and then making a few comments on some open issues.

1. The probe limit and beyond: much of our discussion on the soft theorem has been carried out in the probe limit where one of the objects is much heavier than the other. This ensures that the total amount of radiation emitted during the scattering is small compared to the mass of the lighter object. However there are other ways to ensure this, e.g. by keeping the impact parameter $b$ to be larger than the Schwarzschild radii of the objects, as in the example of sections 7.2.1 and 7.2.2. Soft theorem can be used to compute classical gravity wave radiation during such cases as well - the only change is that we now have to use the soft factors given in (3.2) and (3.6) instead of (3.24) and (3.26). Alternatively we can use (3.24), (3.26) but allow the sum over $a$ to run over all external states so that the additional terms vanish by momentum conservation. The soft expansion will now be an expansion in powers of $\omega b$.

If we take the external objects to have masses of the same order and allow them to come within a distance of the order of their Schwarzschild radii, then the total radiated energy does not remain small compared to the energies of the incident objects and we can no longer ignore the contribution of the 'hard gravitons' carrying this energy to the soft factors given in (3.2), (3.6), (6.5). Inclusion of these factors will require detailed knowledge of the spectrum of these gravitons in the final state.

2. Nature of interactions: our result does not depend on the nature of the interaction that takes place during the scattering process - it need not be only electromagnetic or gravitational.

3. Multi-body scattering: our analysis can also be applied to multi-body scattering. For example if some object(s) breaks apart into several pieces during the scattering process due to some internal force, we can still compute the soft radiation by knowing 
the initial states before the scattering and the final states after the scattering, without any detailed knowledge of the process that broke the object apart during the scattering process.

4. Hidden assumptions: the derivation of soft theorem in generic theories of gravity, given in [76, 77], implicitly assumed that the mass spectrum of particles in the theory is discrete. In the case of continuous spectrum the emission or absorption of soft radiation could take place via a series of transitions between real intermediate states instead of via the virtual intermediate states as considered in $[76,77]$. For classical objects this assumption of discrete spectrum breaks down since the spectrum becomes almost continuous. However we do not expect emission of soft gravity waves to cause transition between the internal states of the classical object, and therefore the fact that the spectrum is not discrete should not affect the derivation of the soft theorem. A possible exception arises when the scatterer is a black hole. In this case some of the gravity waves emitted during the scattering could be absorbed by the black hole, changing the internal state of the black hole. Can this affect our analysis? Soft radiations of wave-length $\omega^{-1}$ are expected to be produced at a distance of order $\omega^{-1}$ from the scatterer. For $D=4$ the solid angle subtended by the scatterer of linear size $a$ to a point at a distance $\omega^{-1}$ is of order $a^{2} \omega^{2}$. This is a subsubleading effect and is of the same order as the non-universal terms in the soft theorem. In higher dimensions the effect is even smaller. Therefore we do not expect our results up to subleading order (and the universal part at the subsubleading order) to be affected due to the absorption of the soft radiation by the black hole.

5. Observation: given the observation of gravitational wave at LIGO [104], one natural question is whether we can actually test soft graviton theorem from the observation of gravitational waves. Unfortunately the direct application of soft theorem requires us to consider a scattering event instead of the merger of a bound pair of objects that is the common source of gravitational waves. The most likely event is the fusion of two massive objects as discussed in section 5 or their scattering at close encounter discussed in section 3. However since the soft factor is small when the relative velocity between the incoming objects is small, an appreciable soft radiation will require reasonably large initial relative velocity between the two objects when they are far away from each other, and at the same time their trajectories must come close to each other so that there is appreciable amount of gravitational radiation. Such events are rare. If we want to go beyond the leading order, we also need to understand properly the infrared modification of the soft theorem in four dimensions. Despite these difficulties it is not inconceivable that soft graviton theorem may be tested in actual gravitational wave experiments in the distant future.

\section{Acknowledgments}

We wish to thank K.G. Arun, Sayantani Bhattacharyya, Miguel Campiglia, Gary Horowitz, Sachin Jain, Shilpa Kastha, Prahar Mitra, Siddharth Prabhu, Madhusudan Raman, K.P. 
Yogendran and Yogesh Srivastava for useful discussions and K.G. Arun for pointing out very useful references. A.S. would like to acknowledge the hospitality of IMSc, Chennai where this work was initiated, ICTS Bangalore where this work was nearing completion and KITP, Santa Barbara for a visit during the course of this work during which the research was supported in part by the National Science Foundation under Grant No. NSF PHY-1125915. A.S. would also like to thank the theory group at NISER, Bhubaneswar for hospitality during a visit when this work was presented. A.L. would like to thank IIT Gandhinagar for their hospitality during the course of this work and ICTS Bangalore during the final stages of this work. Work of A.L is supported in part by Ramanujan Fellowship. The work of A.S. was supported in part by the J. C. Bose fellowship of the Department of Science and Technology, India.

\section{A Alternative analysis of the test of soft photon theorem in four dimen- sions}

In this appendix we shall recall the result of [79] for classical electromagnetic radiation from particle scattering in four space-time dimensions and compare the result with the prediction of the soft photon theorem. From eq. (15.1) of [79] we get the formula for the total energy radiated in the frequency range between $\omega$ and $\omega(1+\delta)$, carrying polarization $\varepsilon$, within a solid angle $\Delta \Omega$ around the direction labelled by $\hat{n}$ :

$$
\frac{q^{2}}{16 \pi^{3}} \omega \delta \Delta \Omega\left|\int \frac{d}{d t}\left[\frac{\vec{\varepsilon} \cdot\{\hat{n} \times(\hat{n} \times \vec{\beta})\}}{1-\hat{n} \cdot \vec{\beta}}\right] e^{i \omega(t-\hat{n} \cdot \vec{r}(t))} d t\right|^{2} .
$$

Here $\vec{r}(t)$ is the location of the probe at time $t$ and $\vec{\beta}=d \vec{r} / d t$ is the velocity. The polarization four vector has been taken to be of the form $(0, \vec{\varepsilon})$ with $k^{\mu} \varepsilon_{\mu}=-\omega \hat{n} \cdot \vec{\varepsilon}=0$. In writing (A.1) we have taken into account the factor of $4 \pi$ mentioned in footnote 5 . Comparing this with (2.7) for $D=4$ we see that the soft factor should be given by

$$
\bar{S}_{\mathrm{em}}(\varepsilon, k)=e^{i \alpha} \frac{q}{\omega} \int \frac{d}{d t}\left[\frac{\vec{\varepsilon} \cdot\{\hat{n} \times(\hat{n} \times \vec{\beta})\}}{1-\hat{n} \cdot \vec{\beta}}\right] e^{i \omega(t-\hat{n} \cdot \vec{r}(t))} d t,
$$

where $e^{i \alpha}$ is a phase that will be fixed by comparing this with the leading soft factor. The bar on $S_{\mathrm{em}}$ indicates that this is the soft factor computed from the classical radiation formula. Using the gauge condition $\vec{\varepsilon} \cdot \hat{n}=0$, we can express this as

$$
\bar{S}_{\mathrm{em}}(\varepsilon, k)=-e^{i \alpha} \frac{q}{\omega} \int \frac{d}{d t}\left[\frac{\vec{\varepsilon} \cdot \vec{\beta}}{1-\hat{n} \cdot \vec{\beta}}\right] e^{i \omega(t-\hat{n} \cdot \vec{r}(t))} d t .
$$

We now expand $e^{i \omega(t-\hat{n} \cdot \vec{r}(t))}$ to first order in $\omega$ and integrate by parts the integral over $t$. This gives

$$
\bar{S}_{\mathrm{em}}(\varepsilon, k)=-e^{i \alpha} \frac{q}{\omega}\left[\frac{\vec{\varepsilon} \cdot \vec{\beta}}{1-\hat{n} \cdot \vec{\beta}}\{1+i \omega(t-\hat{n} \cdot \vec{r}(t))\}\right]_{t_{-}}^{t_{+}}+e^{i \alpha} \frac{q}{\omega} \int_{t_{-}}^{t_{+}} d t \frac{\vec{\varepsilon} \cdot \vec{\beta}}{1-\hat{n} \cdot \vec{\beta}} i \omega(1-\hat{n} \cdot \vec{\beta})
$$


where we have to take $t_{ \pm} \rightarrow \pm \infty$ limit at the end of the calculation. We now note that using the relation $\vec{\beta}=d \vec{r} / d t$ the second term can be integrated explicitly, and we can express (A.4) as

$$
\begin{aligned}
\bar{S}_{\mathrm{em}}(\varepsilon, k)= & -e^{i \alpha} \frac{q}{\omega}\left[\frac{\vec{\varepsilon} \cdot \vec{\beta}}{1-\hat{n} \cdot \vec{\beta}}\{1+i \omega(t-\hat{n} \cdot \vec{r}(t))\}\right]_{t_{-}}^{t_{+}}+e^{i \alpha} \frac{q}{\omega} i \omega[\vec{\varepsilon} \cdot \vec{r}]_{t_{-}}^{t_{+}} \\
= & -e^{i \alpha} q \omega^{-1}\left[\frac{\vec{\varepsilon} \cdot \vec{\beta}_{+}}{1-\hat{n} \cdot \vec{\beta}_{+}}-\frac{\vec{\varepsilon} \cdot \vec{\beta}_{-}}{1-\hat{n} \cdot \vec{\beta}_{-}}\right] \\
& +i q e^{i \alpha}\left[\frac{1}{1-\hat{n} \cdot \vec{\beta}_{+}}\left\{-\vec{\varepsilon} \cdot \vec{\beta}_{+} t_{+}+\vec{\varepsilon} \cdot \vec{\beta}_{+} \hat{n} \cdot \vec{r}\left(t_{+}\right)+\vec{\varepsilon} \cdot \vec{r}\left(t_{+}\right)-\hat{n} \cdot \vec{\beta}_{+} \vec{\varepsilon} \cdot \vec{r}\left(t_{+}\right)\right\}\right] \\
& -i q e^{i \alpha}\left[\frac{1}{1-\hat{n} \cdot \vec{\beta}_{-}}\left\{-\vec{\varepsilon} \cdot \vec{\beta}_{-} t_{-}+\vec{\varepsilon} \cdot \vec{\beta}_{-} \hat{n} \cdot \vec{r}\left(t_{-}\right)+\vec{\varepsilon} \cdot \vec{r}\left(t_{-}\right)-\hat{n} \cdot \overrightarrow{\beta_{-}} \vec{\varepsilon} \cdot \vec{r}\left(t_{-}\right)\right\}\right] .
\end{aligned}
$$

Using covariant notation,

$$
V_{ \pm}=V_{ \pm}^{0}\left(1, \vec{\beta}_{ \pm}\right), \quad V_{ \pm}^{0}=\left(1-\vec{\beta}_{ \pm}^{2}\right)^{-1 / 2}, \quad r_{ \pm}=\left(t_{ \pm}, \vec{r}_{ \pm}\right), \quad k=-\omega(1, \hat{n}),
$$

(A.5) may be written as

$$
\bar{S}_{\mathrm{em}}=e^{i \alpha}\left[-q\left\{\frac{\varepsilon \cdot V_{+}}{k \cdot V_{+}}-\frac{\varepsilon \cdot V_{-}}{k \cdot V_{-}}\right\}-\frac{i q}{k \cdot V_{+}}\left\{\varepsilon \cdot V_{+} k \cdot r_{+}-\varepsilon \cdot r_{+} k \cdot V_{+}\right\}+\frac{i q}{k \cdot V_{-}}\left\{\varepsilon \cdot V_{-} k \cdot r_{-}-\varepsilon \cdot r_{-} k \cdot V_{-}\right\}\right] .
$$

Upon using (7.16) this becomes identical to the right hand side of (7.19) for $\alpha=0$. Therefore we see that $\bar{S}_{\mathrm{em}}=S_{\mathrm{em}}$.

Open Access. This article is distributed under the terms of the Creative Commons Attribution License (CC-BY 4.0), which permits any use, distribution and reproduction in any medium, provided the original author(s) and source are credited.

\section{References}

[1] M. Gell-Mann and M.L. Goldberger, Scattering of low-energy photons by particles of spin 1/2, Phys. Rev. 96 (1954) 1433 [inSPIRE].

[2] F.E. Low, Bremsstrahlung of very low-energy quanta in elementary particle collisions, Phys. Rev. 110 (1958) 974 [INSPIRE].

[3] S. Saito, Low-energy theorem for Compton scattering, Phys. Rev. 184 (1969) 1894 [INSPIRE].

[4] T.H. Burnett and N.M. Kroll, Extension of the low soft photon theorem, Phys. Rev. Lett. 20 (1968) 86 [INSPIRE].

[5] J.S. Bell and R. Van Royen, On the low-burnett-kroll theorem for soft-photon emission, Nuovo Cim. A 60 (1969) 62 [InSPIRE].

[6] V. Del Duca, High-energy Bremsstrahlung Theorems for Soft Photons, Nucl. Phys. B 345 (1990) 369 [INSPIRE]. 
[7] S. Weinberg, Photons and Gravitons in s Matrix Theory: Derivation of Charge Conservation and Equality of Gravitational and Inertial Mass, Phys. Rev. 135 (1964) B1049 [INSPIRE].

[8] S. Weinberg, Infrared photons and gravitons, Phys. Rev. 140 (1965) B516 [InSPIRE].

[9] D.J. Gross and R. Jackiw, Low-Energy Theorem for Graviton Scattering, Phys. Rev. 166 (1968) 1287 [INSPIRE].

[10] R. Jackiw, Low-Energy Theorems for Massless Bosons: Photons and Gravitons, Phys. Rev. 168 (1968) 1623 [INSPIRE].

[11] M. Ademollo et al., Soft Dilations and Scale Renormalization in Dual Theories, Nucl. Phys. B 94 (1975) 221 [INSPIRE].

[12] J.A. Shapiro, On the Renormalization of Dual Models, Phys. Rev. D 11 (1975) 2937 [INSPIRE].

[13] A. Strominger, On BMS Invariance of Gravitational Scattering, JHEP 07 (2014) 152 [arXiv:1312.2229] [INSPIRE].

[14] T. He, V. Lysov, P. Mitra and A. Strominger, BMS supertranslations and Weinberg's soft graviton theorem, JHEP 05 (2015) 151 [arXiv: 1401.7026] [INSPIRE].

[15] M. Campiglia and A. Laddha, Asymptotic symmetries and subleading soft graviton theorem, Phys. Rev. D 90 (2014) 124028 [arXiv:1408.2228] [INSPIRE].

[16] A. Strominger and A. Zhiboedov, Gravitational Memory, BMS Supertranslations and Soft Theorems, JHEP 01 (2016) 086 [arXiv:1411.5745] [INSPIRE].

[17] M. Campiglia and A. Laddha, New symmetries for the Gravitational S-matrix, JHEP 04 (2015) 076 [arXiv: 1502.02318] [INSPIRE].

[18] M. Campiglia and A. Laddha, Asymptotic symmetries of QED and Weinberg's soft photon theorem, JHEP 07 (2015) 115 [arXiv: 1505.05346] [INSPIRE].

[19] S.G. Avery and B.U.W. Schwab, Burg-Metzner-Sachs symmetry, string theory and soft theorems, Phys. Rev. D 93 (2016) 026003 [arXiv: 1506.05789] [inSPIRE].

[20] M. Campiglia and A. Laddha, Asymptotic symmetries of gravity and soft theorems for massive particles, JHEP 12 (2015) 094 [arXiv:1509.01406] [INSPIRE].

[21] M. Campiglia and A. Laddha, Sub-subleading soft gravitons: New symmetries of quantum gravity?, Phys. Lett. B 764 (2017) 218 [arXiv:1605.09094] [INSPIRE].

[22] M. Campiglia and A. Laddha, Subleading soft photons and large gauge transformations, JHEP 11 (2016) 012 [arXiv: 1605.09677] [INSPIRE].

[23] M. Campiglia and A. Laddha, Sub-subleading soft gravitons and large diffeomorphisms, JHEP 01 (2017) 036 [arXiv: 1608.00685] [INSPIRE].

[24] E. Conde and P. Mao, BMS Supertranslations and Not So Soft Gravitons, JHEP 05 (2017) 060 [arXiv: 1612.08294] [INSPIRE].

[25] T. He, D. Kapec, A.-M. Raclariu and A. Strominger, Loop-Corrected Virasoro Symmetry of $4 D$ Quantum Gravity, JHEP 08 (2017) 050 [arXiv:1701.00496] [INSPIRE].

[26] M. Asorey, A.P. Balachandran, F. Lizzi and G. Marmo, Equations of Motion as Constraints: Superselection Rules, Ward Identities, JHEP 03 (2017) 136 [arXiv:1612.05886] [INSPIRE]. 
[27] A. Strominger, Lectures on the Infrared Structure of Gravity and Gauge Theory, arXiv: 1703.05448 [INSPIRE].

[28] A. Laddha and P. Mitra, Asymptotic Symmetries and Subleading Soft Photon Theorem in Effective Field Theories, JHEP 05 (2018) 132 [arXiv:1709.03850] [inSPIRE].

[29] D. Kapec and P. Mitra, A d-Dimensional Stress Tensor for Mink ${ }_{d+2}$ Gravity, JHEP 05 (2018) 186 [arXiv:1711.04371] [INSPIRE].

[30] H. Anupam, A. Kundu and A. Laddha, work in progress.

[31] C.D. White, Factorization Properties of Soft Graviton Amplitudes, JHEP 05 (2011) 060 [arXiv:1103.2981] [INSPIRE].

[32] F. Cachazo and A. Strominger, Evidence for a New Soft Graviton Theorem, arXiv: 1404.4091 [INSPIRE].

[33] E. Casali, Soft sub-leading divergences in Yang-Mills amplitudes, JHEP 08 (2014) 077 [arXiv: 1404.5551] [INSPIRE].

[34] B.U.W. Schwab and A. Volovich, Subleading Soft Theorem in Arbitrary Dimensions from Scattering Equations, Phys. Rev. Lett. 113 (2014) 101601 [arXiv:1404.7749] [INSPIRE].

[35] Z. Bern, S. Davies and J. Nohle, On Loop Corrections to Subleading Soft Behavior of Gluons and Gravitons, Phys. Rev. D 90 (2014) 085015 [arXiv:1405.1015] [INSPIRE].

[36] S. He, Y.-t. Huang and C. Wen, Loop Corrections to Soft Theorems in Gauge Theories and Gravity, JHEP 12 (2014) 115 [arXiv:1405.1410] [INSPIRE].

[37] A.J. Larkoski, Conformal Invariance of the Subleading Soft Theorem in Gauge Theory, Phys. Rev. D 90 (2014) 087701 [arXiv:1405.2346] [INSPIRE].

[38] F. Cachazo and E.Y. Yuan, Are Soft Theorems Renormalized?, arXiv:1405.3413 [inSPIRE].

[39] N. Afkhami-Jeddi, Soft Graviton Theorem in Arbitrary Dimensions, arXiv:1405.3533 [INSPIRE].

[40] J. Broedel, M. de Leeuw, J. Plefka and M. Rosso, Constraining subleading soft gluon and graviton theorems, Phys. Rev. D 90 (2014) 065024 [arXiv:1406.6574] [INSPIRE].

[41] Z. Bern, S. Davies, P. Di Vecchia and J. Nohle, Low-Energy Behavior of Gluons and Gravitons from Gauge Invariance, Phys. Rev. D 90 (2014) 084035 [arXiv:1406.6987] [INSPIRE].

[42] C.D. White, Diagrammatic insights into next-to-soft corrections, Phys. Lett. B 737 (2014) 216 [arXiv: 1406.7184] [INSPIRE].

[43] M. Zlotnikov, Sub-sub-leading soft-graviton theorem in arbitrary dimension, JHEP 10 (2014) 148 [arXiv: 1407.5936] [INSPIRE].

[44] C. Kalousios and F. Rojas, Next to subleading soft-graviton theorem in arbitrary dimensions, JHEP 01 (2015) 107 [arXiv: 1407.5982] [INSPIRE].

[45] Y.-J. Du, B. Feng, C.-H. Fu and Y. Wang, Note on Soft Graviton theorem by KLT Relation, JHEP 11 (2014) 090 [arXiv: 1408.4179] [INSPIRE].

[46] D. Bonocore, E. Laenen, L. Magnea, L. Vernazza and C.D. White, The method of regions and next-to-soft corrections in Drell-Yan production, Phys. Lett. B 742 (2015) 375 [arXiv: 1410.6406] [INSPIRE]. 
[47] A. Sabio Vera and M.A. Vazquez-Mozo, The Double Copy Structure of Soft Gravitons, JHEP 03 (2015) 070 [arXiv: 1412.3699] [InSPIRE].

[48] F. Cachazo, S. He and E.Y. Yuan, New Double Soft Emission Theorems, Phys. Rev. D 92 (2015) 065030 [arXiv: 1503.04816] [INSPIRE].

[49] A.E. Lipstein, Soft Theorems from Conformal Field Theory, JHEP 06 (2015) 166 [arXiv: 1504.01364] [INSPIRE].

[50] S.D. Alston, D.C. Dunbar and W.B. Perkins, $n$-point amplitudes with a single negative-helicity graviton, Phys. Rev. D 92 (2015) 065024 [arXiv:1507.08882] [INSPIRE].

[51] Y.-t. Huang and C. Wen, Soft theorems from anomalous symmetries, JHEP 12 (2015) 143 [arXiv: 1509.07840] [INSPIRE].

[52] J. Rao and B. Feng, Note on Identities Inspired by New Soft Theorems, JHEP 04 (2016) 173 [arXiv: 1604.00650] [INSPIRE].

[53] S. He, Z. Liu and J.-B. Wu, Scattering Equations, Twistor-string Formulas and Double-soft Limits in Four Dimensions, JHEP 07 (2016) 060 [arXiv: 1604.02834] [INSPIRE].

[54] F. Cachazo, P. Cha and S. Mizera, Extensions of Theories from Soft Limits, JHEP 06 (2016) 170 [arXiv: 1604.03893] [INSPIRE].

[55] A.P. Saha, Double Soft Theorem for Perturbative Gravity, JHEP 09 (2016) 165 [arXiv: 1607.02700] [INSPIRE].

[56] A. Luna, S. Melville, S.G. Naculich and C.D. White, Next-to-soft corrections to high energy scattering in QCD and gravity, JHEP 01 (2017) 052 [arXiv:1611.02172] [INSPIRE].

[57] H. Elvang, C.R.T. Jones and S.G. Naculich, Soft Photon and Graviton Theorems in Effective Field Theory, Phys. Rev. Lett. 118 (2017) 231601 [arXiv:1611.07534] [InSPIRE].

[58] C. Cheung, K. Kampf, J. Novotny, C.-H. Shen and J. Trnka, A Periodic Table of Effective Field Theories, JHEP 02 (2017) 020 [arXiv:1611.03137] [INSPIRE].

[59] A.P. Saha, Double soft limit of the graviton amplitude from the Cachazo-He-Yuan formalism, Phys. Rev. D 96 (2017) 045002 [arXiv:1702.02350] [INSPIRE].

[60] B.U.W. Schwab, Subleading Soft Factor for String Disk Amplitudes, JHEP 08 (2014) 062 [arXiv:1406.4172] [INSPIRE].

[61] M. Bianchi, S. He, Y.-t. Huang and C. Wen, More on Soft Theorems: Trees, Loops and Strings, Phys. Rev. D 92 (2015) 065022 [arXiv:1406.5155] [INSPIRE].

[62] B.U.W. Schwab, A Note on Soft Factors for Closed String Scattering, JHEP 03 (2015) 140 [arXiv:1411.6661] [INSPIRE].

[63] P. Di Vecchia, R. Marotta and M. Mojaza, Soft theorem for the graviton, dilaton and the Kalb-Ramond field in the bosonic string, JHEP 05 (2015) 137 [arXiv:1502.05258] [INSPIRE].

[64] T. Klose, T. McLoughlin, D. Nandan, J. Plefka and G. Travaglini, Double-Soft Limits of Gluons and Gravitons, JHEP 07 (2015) 135 [arXiv: 1504.05558] [INSPIRE].

[65] A. Volovich, C. Wen and M. Zlotnikov, Double Soft Theorems in Gauge and String Theories, JHEP 07 (2015) 095 [arXiv: 1504.05559] [INSPIRE].

[66] M. Bianchi and A.L. Guerrieri, On the soft limit of open string disk amplitudes with massive states, JHEP 09 (2015) 164 [arXiv:1505.05854] [INSPIRE]. 
[67] P. Di Vecchia, R. Marotta and M. Mojaza, Double-soft behavior for scalars and gluons from string theory, JHEP 12 (2015) 150 [arXiv:1507.00938] [INSPIRE].

[68] A.L. Guerrieri, Soft behavior of string amplitudes with external massive states, Nuovo Cim. C 39 (2016) 221 [arXiv:1507.08829] [INSPIRE].

[69] P. Di Vecchia, R. Marotta and M. Mojaza, Soft Theorems from String Theory, Fortsch. Phys. 64 (2016) 389 [arXiv:1511.04921] [INSPIRE].

[70] M. Bianchi and A.L. Guerrieri, On the soft limit of closed string amplitudes with massive states, Nucl. Phys. B 905 (2016) 188 [arXiv:1512.00803] [inSPIRE].

[71] M. Bianchi and A.L. Guerrieri, On the soft limit of tree-level string amplitudes, arXiv: 1601.03457 [INSPIRE].

[72] P. Di Vecchia, R. Marotta and M. Mojaza, Subsubleading soft theorems of gravitons and dilatons in the bosonic string, JHEP 06 (2016) 054 [arXiv: 1604.03355] [INSPIRE].

[73] P. Di Vecchia, R. Marotta and M. Mojaza, Soft behavior of a closed massless state in superstring and universality in the soft behavior of the dilaton, JHEP 12 (2016) 020 [arXiv:1610.03481] [INSPIRE].

[74] A. Sen, Soft Theorems in Superstring Theory, JHEP 06 (2017) 113 [arXiv:1702.03934] [INSPIRE].

[75] P. Di Vecchia, R. Marotta and M. Mojaza, Double-soft behavior of the dilaton of spontaneously broken conformal invariance, JHEP 09 (2017) 001 [arXiv:1705.06175] [INSPIRE].

[76] A. Sen, Subleading Soft Graviton Theorem for Loop Amplitudes, JHEP 11 (2017) 123 [arXiv: 1703.00024] [INSPIRE].

[77] A. Laddha and A. Sen, Sub-subleading Soft Graviton Theorem in Generic Theories of Quantum Gravity, JHEP 10 (2017) 065 [arXiv:1706.00759] [INSPIRE].

[78] S. Chakrabarti, S.P. Kashyap, B. Sahoo, A. Sen and M. Verma, Subleading Soft Theorem for Multiple Soft Gravitons, JHEP 12 (2017) 150 [arXiv:1707.06803] [INSPIRE].

[79] J.D. Jackson, Classical Electrodynamics, John Wiley and Sons.

[80] J. Ware, R. Saotome and R. Akhoury, Construction of an asymptotic S matrix for perturbative quantum gravity, JHEP 10 (2013) 159 [arXiv: 1308.6285] [INSPIRE].

[81] S. Hollands, A. Ishibashi and R.M. Wald, BMS Supertranslations and Memory in Four and Higher Dimensions, Class. Quant. Grav. 34 (2017) 155005 [arXiv: 1612.03290] [INSPIRE].

[82] D. Garfinkle, S. Hollands, A. Ishibashi, A. Tolish and R.M. Wald, The Memory Effect for Particle Scattering in Even Spacetime Dimensions, Class. Quant. Grav. 34 (2017) 145015 [arXiv: 1702.00095] [INSPIRE].

[83] G. Satishchandran and R.M. Wald, Memory effect for particle scattering in odd spacetime dimensions, Phys. Rev. D 97 (2018) 024036 [arXiv:1712.00873] [INSPIRE].

[84] M. Pate, A.-M. Raclariu and A. Strominger, Gravitational Memory in Higher Dimensions, JHEP 06 (2018) 138 [arXiv:1712.01204] [INSPIRE].

[85] S. Weinberg, Gravitation and Cosmology: Principles and Applications of the General Theory of Relativity, John Wiley and Sons. 
[86] S. Pasterski, A. Strominger and A. Zhiboedov, New Gravitational Memories, JHEP 12 (2016) 053 [arXiv: 1502.06120] [INSPIRE].

[87] W.D. Goldberger and I.Z. Rothstein, An effective field theory of gravity for extended objects, Phys. Rev. D 73 (2006) 104029 [hep-th/0409156] [InSPIRE].

[88] W.D. Goldberger and I.Z. Rothstein, Towers of Gravitational Theories, Gen. Rel. Grav. 38 (2006) 1537 [hep-th/0605238] [INSPIRE].

[89] R.A. Porto, Post-Newtonian corrections to the motion of spinning bodies in NRGR, Phys. Rev. D 73 (2006) 104031 [gr-qc/0511061] [INSPIRE].

[90] R.A. Porto, The effective field theorist's approach to gravitational dynamics, Phys. Rept. 633 (2016) 1 [arXiv: 1601.04914] [INSPIRE].

[91] D. Kapec, V. Lysov, S. Pasterski and A. Strominger, Higher-Dimensional Supertranslations and Weinberg's Soft Graviton Theorem, arXiv:1502.07644 [INSPIRE].

[92] M. Pate, A.-M. Raclariu and A. Strominger, Color Memory: A Yang-Mills Analog of Gravitational Wave Memory, Phys. Rev. Lett. 119 (2017) 261602 [arXiv:1707.08016] [INSPIRE].

[93] Ya. B. Zeldovich and A.G. Polnarev, Radiation of gravitational waves by a cluster of superdense stars, Sov. Astron. 18 (1974) 17.

[94] V.B. Braginsky and L.P. Grishchuk, Kinematic Resonance and Memory Effect in Free Mass Gravitational Antennas, Sov. Phys. JETP 62 (1985) 427 [INSPIRE].

[95] V.B. Braginsky and K.S. Thorne, Gravitational-wave bursts with memory and experimental prospects, Nature $\mathbf{3 2 7}$ (1987) 123.

[96] M. Ludvigsen, Geodesic Deviation At Null Infinity And The Physical Effects Of Very Long Wave Gravitational Radiation, Gen. Rel. Grav. 21 (1989) 1205 [INSPIRE].

[97] V. Cardoso, O.J.C. Dias and J.P.S. Lemos, Gravitational radiation in D-dimensional space-times, Phys. Rev. D 67 (2003) 064026 [hep-th/0212168] [INSPIRE].

[98] W.D. Goldberger and A.K. Ridgway, Radiation and the classical double copy for color charges, Phys. Rev. D 95 (2017) 125010 [arXiv:1611.03493] [INSPIRE].

[99] W.D. Goldberger, S.G. Prabhu and J.O. Thompson, Classical gluon and graviton radiation from the bi-adjoint scalar double copy, Phys. Rev. D 96 (2017) 065009 [arXiv:1705.09263] [INSPIRE].

[100] W.D. Goldberger, J. Li and S.G. Prabhu, Spinning particles, axion radiation and the classical double copy, Phys. Rev. D 97 (2018) 105018 [arXiv:1712.09250] [INSPIRE].

[101] P.C. Peters, Relativistic gravitational bremsstrahlung, Phys. Rev. D 1 (1970) 1559 [INSPIRE].

[102] E. Berti, M. Cavaglia and L. Gualtieri, Gravitational energy loss in high-energy particle collisions: Ultrarelativistic plunge into a multidimensional black hole, Phys. Rev. D 69 (2004) 124011 [hep-th/0309203] [INSPIRE].

[103] D. Gal'tsov, P. Spirin and T.N. Tomaras, Gravitational bremsstrahlung in ultra-planckian collisions, JHEP 01 (2013) 087 [arXiv:1210.6976] [INSPIRE].

[104] Virgo and LigO Scientific collaborations, B.P. Abbott et al., Observation of Gravitational Waves from a Binary Black Hole Merger, Phys. Rev. Lett. 116 (2016) 061102 [arXiv: 1602.03837] [INSPIRE]. 